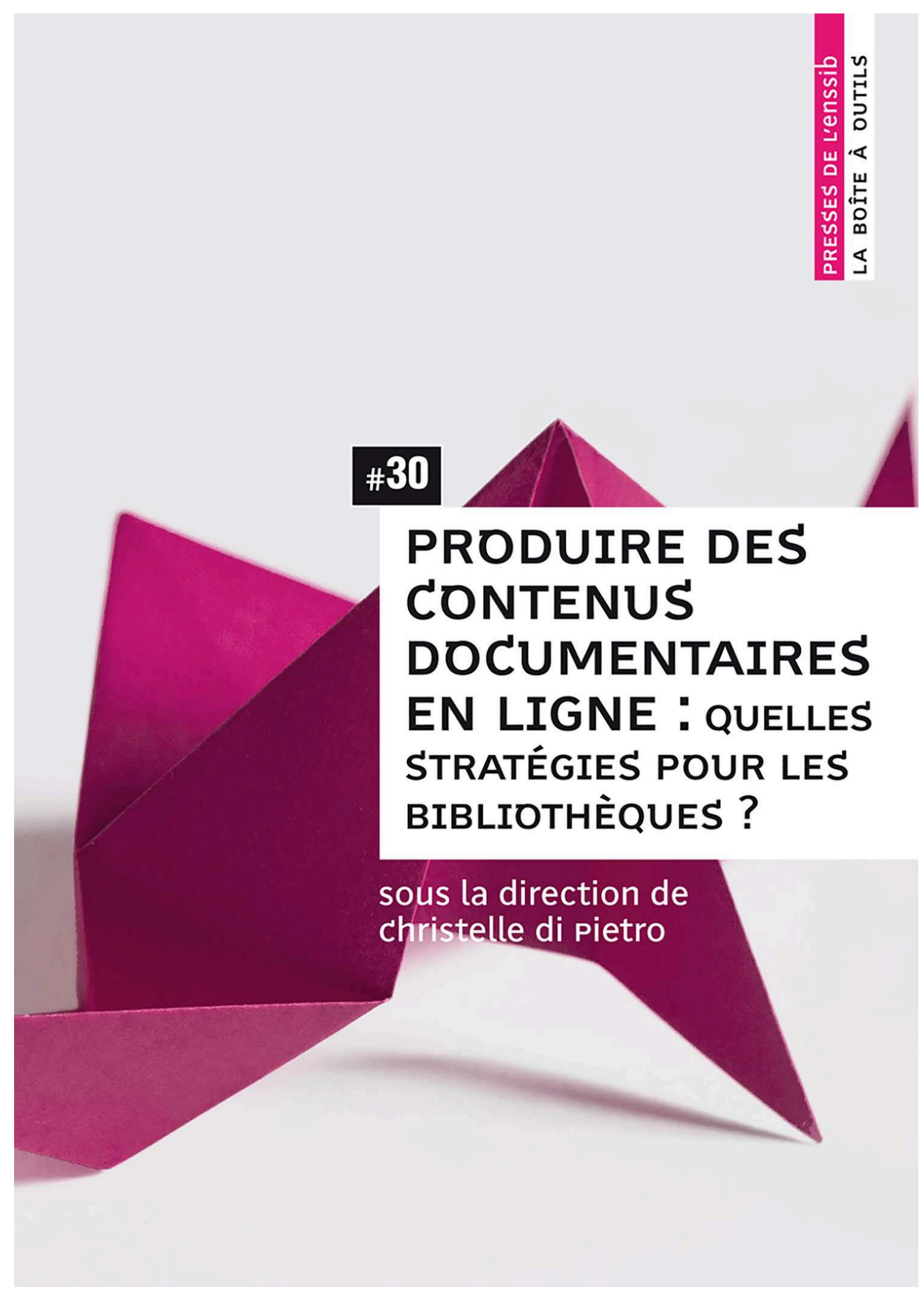




\section{Produire des contenus documentaires en ligne}

Quelles stratégies pour les bibliothèques?

\section{Christelle Di Pietro (dir.)}

DOI : 10.4000/books.pressesenssib.2814

Éditeur: Presses de l'enssib

Lieu d'édition : Villeurbanne

Année d'édition : 2014

Date de mise en ligne : 10 décembre 2018

Collection : La Boîte à outils

ISBN électronique : 9782375460603

\section{Qboooks}

http://books.openedition.org

\section{Édition imprimée}

Date de publication : 1 janvier 2014

ISBN : 9791091281379

Nombre de pages : 188

\section{Référence électronique}

DI PIETRO, Christelle (dir.). Produire des contenus documentaires en ligne : Quelles stratégies pour les bibliothèques ? Nouvelle édition [en ligne]. Villeurbanne : Presses de l'enssib, 2014 (généré le 22 février 2021). Disponible sur Internet : <http://books.openedition.org/pressesenssib/2814>. ISBN :

9782375460603. DOI : https://doi.org/10.4000/books.pressesenssib.2814.

(C) Presses de l'enssib, 2014

Conditions d'utilisation

http://www.openedition.org/6540 
\#01

PRODUIRE DES

CONTENUS

DOCUMENTAIRES

EN LIGNE : QUELLES

STRATÉGIES POUR LES

BIBLIOTHĖQUES ?

sous la direction de christelle Di pietro 


\section{PRODUIRE DES CONTENUS DOCUMENTAIRES EN LIGNE : QUELLES STRATÉGIES POUR LES BIBLIOTHĖQUES ?}

Plébiscités par les publics, les services d'information se multiplient et se diversifient. La gestion des collections en bibliothèque implique désormais leur exploitation et la réappropriation de ces contenus pour élaborer toute une gamme de produits documentaires.

Nouvelles compétences du professionnel, renouvellement des formes de collaboration, aussi bien sur les aspects techniques, juridiques que méthodologiques et rédactionnels, ce volume investit de façon inédite le champ de l'Internet de contenus pour tous les types de publics.

Le plan de l'ouvrage s'articule autour de quatre parties : exploiter les collections et repenser les accès en ligne, les produits documentaires de synthèse : curation et production, produire en co-construction et en réseau, et enfin, les outils et le droit.

Dans ce volume collectif, des bibliothécaires, documentalistes, formateurs, juriste, community manager..., fournissent des axes de travail opérationnels, et suggèrent des pistes de réflexion-action à travers un panorama des services proposés par des établissements au rayonnement territorial comme universitaire.

Christelle Di Pietro est conservatrice des bibliothèques.

Mots clés : agrégateurs, bibliothèques numériques, curation, compétences documentaires, droit de l'information, e-books, gestion de l'information, recherche documentaire, réseaux sociaux 
Ont contribué à cet ouvrage:

Michèle Battisti

Pierre Bournerie

Isabelle Breuil

Jocelyne Deschaux*

Christelle Di Pietro

Lionel Dujol

Bruno Essard-Budaïl

Magali Haettiger*

Patrick Hernebring*
Gonzague Gauthier

Magalie Le Gall

Dominique Macé

Sébastien Magro

Nathalie Nosny

Jérôme Pouchol

Isabelle Rouquet

Carole Tilbian

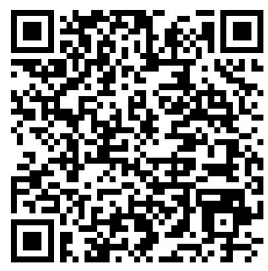

* Des bonus numériques sont disponibles sur le site des presses de l'enssib: < http://www.enssib.fr/presses/catalogue/ produire-des-contenus-documentaires-en-ligne-30 > ; deux galeries d'images extraites l'une de Numelyo et l'autre du Fonds Trutat.

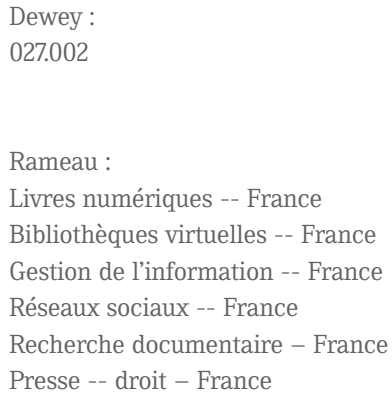

Notice rédigée par la bibliothèque de l'enssib. 


\section{MODE D'EMPLOI}

par Christelle Di Pietro

\section{UNE NOUVELLE OFFRE DOCUMENTAIRE : LA PRODUCTION DE CONTENUS}

En 2004, Alexandre Serres évoquait déjà pour les documentalistes la transition de la gestion des stocks vers la gestion des flux d'informations ${ }^{1}$. Il dénonçait en particulier la " crise des médiateurs » sur le Web, lieu anarchique de production de l'information où les filtres traditionnels (éditeurs, journalistes, bibliothécaires) étaient remplacés par des producteurs non contrôlés. Ce constat partagé par les professionnels de l'information aboutit à une mutation de la gestion de l'information qui ne se contente plus d'une mise à disposition mais d'une production propre qui tend à structurer les contenus en ligne.

C'est ainsi que l'on assiste à une professionnalisation de la production de contenus en bibliothèque, que l'on peut notamment mesurer à travers l'offre de formation continue qui connaît un franc succès ${ }^{2}$. La prise de conscience des bibliothécaires de la nécessaire exploitation de leurs collections par des actions de médiation n'est bien sûr pas nouvelle ; toutes les actions documentaires développées par les bibliothèques prennent généralement la forme de productions de contenus : bibliographies, coups de cœur, listes d'acquisitions, toutes axées autour de la valorisation des collections. Cependant, l'explosion des contenus en ligne a fait apparaître la nécessité d'appréhender des ressources extérieures et de les proposer au même titre que celles acquises par la bibliothèque.

1. Alexandre Serres, Recherche d'information sur Internet : où en sommes-nous, où allons-nous ? CNDP, juin 2004. [En ligne] : < http://www.cndp.fr/savoirscdi/index.php?id=1052 >.

2. L'enssib et le CRFCB Rhône-Alpes ont uni leurs offres et vont proposer un stage sur ce sujet en décembre 2014 : < http://www.enssib.fr/offre-de-formation/formation-continue/14e29-produiredes-contenus-en-ligne-en-bibliotheque >. 


\section{DE LA GESTION À L'ACTION DOCUMENTAIRE : LES ANNÉES 2000}

On peut identifier au moins deux événements qui ont accéléré ce processus et modélisé une nouvelle forme d'offre. Le premier est le rapprochement des métiers de bibliothécaire et de documentaliste, notamment par une fusion des formations initiales, laquelle a été favorisée par la fin du Certificat d'aptitude aux fonctions de bibliothécaires (CAFB $\left.{ }^{3}, 1951-1994\right)$, permettant ainsi à l'université de proposer des formations communes aux deux corps de métiers. Ces formations reflètent une conception harmonisée des deux professions abordant tout aussi bien la constitution des collections que la production de synthèses documentaires ${ }^{4}$. Cette dichotomie historique, propre à la France - aucun autre pays n'a développé une distinction entre métiers des bibliothèques et métiers de la documentation - a probablement été l'un des principaux freins au développement de produits documentaires adaptés à la demande des usagers par les bibliothécaires autrement que par l'exploitation de l'objet collection. Cantonné à la gestion du stock, le bibliothécaire ne se pense pas comme producteur de contenus, alors que le documentaliste, en sélectionnant, synthétisant et diffusant des informations thématisées à des profils définis d'usagers se pose par essence en médiateur documentaire.

Le second événement est le développement (ou l'explosion !), des services de références en ligne ${ }^{5}$ : en considérant strictement la demande des usagers indépendamment des collections détenues, le bibliothécaire a dû repenser son offre de contenus, et accepter un nouveau rôle de « curateur ${ }^{* 6}$ de l'information. Cette mutation s'est accompagnée de nouvelles compétences, dont l'écriture en ligne et la coordination de la rédaction collaborative.

3. Pour les sigles et acronymes, se reporter à la liste en fin d'ouvrage.

4. Voir le Programme pédagogique national du diplôme universitaire de technologie informationcommunication : <http://cache.media.enseignementsup-recherche.gouv.fr/file/30/15/0/Infocom_262150.pdf $>$.

5. Claire Nguyen (dir.), Mettre en ouvre un service de questions-réponses en ligne, Villeurbanne, Presses de l'enssib, 2010 (coll. La Boîte à outils ; 20).

6. Les termes suivis d'un astérisque (à leur première occurrence) sont définis dans le glossaire en fin d'ouvrage. 
Ainsi, cette boîte à outils peut être lue comme un prolongement de celle dirigée par Xavier Galaup sur la médiation documentaire numérique ${ }^{7}$. Alors que cette dernière introduisait un cadre général à la médiation numérique et s'achevait sur des exemples de productions de contenus dématérialisés, celle-ci reprend la fonction de production en la mettant au centre de l'activité du bibliothécaire. Elle décortique les processus d'éditorialisation à travers la mise en œuvre de chaînes de production complexes qui repensent les métiers des bibliothèques et réinterroge le bibliothécaire comme " journaliste de ses collections ", pour reprendre l'expression de Lionel Dujol ${ }^{8}$. C'est aussi l'occasion de constater le degré de subtilité rédactionnelle et de conception éditoriale auquel parviennent les bibliothèques ou établissements documentaires aujourd'hui.

\section{PRÉSENTATION DE L'OUVRAGE}

Une première partie dresse le cadre général de la production de contenus en ligne actuelle à travers quelques exemples représentatifs d'une exploitation sophistiquée des collections en lien avec la demande des usagers. Lionel Dujol (Bibliothèques de la Communauté d'agglomération Valence Romans Sud Rhône-Alpes) revient d'abord sur le positionnement stratégique des bibliothèques dans la production documentaire et l'impact que ces nouveaux enjeux ont sur les métiers et l'organisation du travail. Je propose ensuite un panorama non exhaustif des produits documentaires qui peuvent être proposés en ligne par les bibliothèques : leurs objectifs, leurs publics et leur conception. Après ces deux contributions de cadrage, suivent trois présentations de produits à la fois représentatifs des trois phases les plus significatives dans le développement des projets (l'expérimentation, la maturité et la stabilisation) et innovants dans leur façon d'exploiter le catalogue et les collections. Carole Tilbian (bibliothèque de

7. Xavier Galaup (dir.), Développer la médiation documentaire numérique, Villeurbanne, Presses de l'enssib, 2012 (coll. La Boîte à outils ; 25). Voir en libre accès sur le site des presses de l'enssib : $<$ mediationdoc.enssib.fr >.

8. Lionel Dujol, « La bibliothèque, un plus pour le web social », La Bibliothèque apprivoisée, 23 février 2011. [En ligne] : < http://labibapprivoisee.wordpress.com/2011/02/23/la-bibliotheque-unplus-pour-le-web-social/ >. 
Science-po Lyon) présente la revue de sommaires collaborative Sign@l, le plus ancien des produits documentaires présentés qui n'a cessé d'évoluer depuis sa création (1983) et a toujours des projets de développement. Isabelle Breuil (bibliothèque universitaire [BU] de Paris 8 ) revient sur le projet d'un guide de recherche méthodologique élaboré à partir des demandes des étudiants pour construire des parcours de découverte adaptés à leurs besoins. Enfin, Pierre Bournerie (bibliothèque municipale [BM] de Fresnes) explique comment ré-exploiter les données de son catalogue en leur associant les métadonnées de la Bibliothèque nationale de France $(\mathrm{BnF})$ pour s'acheminer vers une nouvelle génération de catalogue sémantique*.

Ces trois productions documentaires ont en commun leur mode d'élaboration : elles s'appuient toutes sur des partenariats ou des expertises extérieures qui leur ont apporté des nouvelles compétences et des outils.

La deuxième partie s'intéresse aux produits documentaires de synthèse et à la curation à travers quatre réalisations originales qui constituent autant de moments différents dans les évolutions temporelles des réalisations des bibliothèques. Jérôme Pouchol (Médiathèque intercommunale du Syndicat d'agglomération nouvelle Ouest-Provence, MIOP) revient sur les dossiers documentaires de la MIOP qui mettent à contribution l'ensemble des acquéreurs de la bibliothèque et associent en une seule page plusieurs produits : synthèses de contenus, bibliographies, sitographies, signets, iconographies, adresses et contacts... Dominique Macé (BM de Bagnolet) revient sur les choix des plates-formes d'agrégation et de curation des collections de la bibliothèque de Bagnolet, en association avec des ressources extérieures. Magali Haettiger (BM de Lorient) évoque ensuite la mise en place du processus de numérisation des collections patrimoniales de la Bibliothèque municipale de Lyon (BmL) dans le cadre d'un partenariat avec Google. La contribution traite de l'ensemble du circuit et de la question épineuse de l'exploitation des métadonnées. Enfin, Nathalie Nosny (Bpi) dévoile la mise en œuvre - transversale à l'ensemble de l'établissement - du tout récent webmagazine* de la Bpi (dont la mise en ligne devrait être concomitante à la parution de cet ouvrage), en soulignant les aspects collaboratifs de la production. Comme les précédents, ces projets s'appuient tous sur 
des coopérations internes et/ou externes. Leur particularité est de ne plus seulement exploiter les collections mais de proposer des produits autonomes à forte valeur ajoutée ré-exploitables dans le cadre d'autres productions ou recherches.

Une troisième partie aborde un aspect émergent de la production de contenu : la co-construction avec les usagers. Les deux premières contributions analysent les réalisations d'une BM et d'une BU : deux facettes de la présence des bibliothèques sur les réseaux sociaux. Le premier revient sur la mise en ligne d'un fonds de photographies de la Bibliothèque municipale à vocation régionale (BMVR) de Toulouse sur Flickr par Jocelyne Deschaux et Patrick Hernebring, projet qui a permis d'expérimenter le taguage par les publics de documents patrimoniaux numérisés. Le second décrit la politique rédactionnelle de la BU de sciences de l'université de Versailles SaintQuentin (UVSQ) conduite par Magalie Le Gall sur les pages Facebook. Hors bibliothèques, Gonzague Gauthier (Centre Pompidou) et Sébastien Magro (Musée du Quai Branly), expliquent comment ils interagissent avec leurs publics en favorisant les conditions de leur participation et en impliquant l'ensemble des personnels.

Ces trois contributions pointent les difficultés d'insérer les publics comme des acteurs de dispositifs de contenus et le paradoxe de libérer les interactions tout en les contrôlant.

Cette partie se clôt avec un projet particulier, conduit par Bruno EssardBudail au sein du Centre du livre et de la lecture (CLL) de Poitou-Charentes, qui montre comment, avec des outils peu onéreux mais performants, chaque bibliothèque peut devenir actrice du partage de ses collections en ligne en créant ses propres ebooks.

Il n'aurait pas été possible d'aborder la question des contenus en ligne sans revenir sur les compétences transversales nécessaires à leur élaboration que sont l'écriture sur le Web, l'appréhension de l'environnement juridique et les outils propres. Michèle Battisti (ADBS), à travers une approche pragmatique du droit et de la jurisprudence, revient sur les situations pratiques que peuvent rencontrer les bibliothécaires producteurs de contenus. Isabelle Rouquet (consultante et formatrice) aborde les techniques d'écriture sur le Web, les éléments de la charte de rédaction et le 
référencement. Enfin, Lionel Dujol conclut cet ouvrage par un panorama des outils de production en ligne : bien qu'essentiels, ils ne doivent pas précéder les projets mais être choisis en fonction des objectifs de départ.

Un dernier mot en forme de clin d'œil d'actualité pour souligner une particularité de cet ouvrage ${ }^{9}$ : presque paritaire et féminisé, il a été rédigé par 10 auteures et 7 auteurs, preuve s'il en est que l'appropriation a priori technique de la production de contenus n'est pas une question de genre...

9. À l'image de la collection, également attentive à l'équilibre femmes/hommes dans l'élaboration des sommaires comme dans la responsabilité de coordination des volumes. 


\section{PARTIE I}

\section{EXPLOITER}

LES COLLECTIONS

ET REPENSER LES ACCÈS

EN LIGNE

1. LE POSITIONNEMENT STRATÉGIQUE DES BIBLIOTHĖQUES DANS LA PRODUCTION DE CONTENUS EN LIGNE, QUELS IMPACTS SUR LES MÉTIERS ?

par Lionel Dujol

$++++++++++++++++++++++++++++++++++++++++++++++++++++++$

2. CONTENUS DOCUMENTAIRES EN LIGNE : UNE TYPOLOGIE EN 8 PRODUITS

par Christelle Di Pietro

$++++++++++++++++++++++++++++++++++++++++++++++++++++++$

3. VALORISER LES PÉRIOdIQUES EN SHS : LE MODĖLE COLLABORATIF DE SIGN@L

par Carole Tilbian

$++++++++++++++++++++++++++++++++++++++++++++++++++++$

4. CONCEVOIR UNE PLATE-FORME D'AUTOFORMATION À LA RECHERCHE DOCUMENTAIRE : ARBRADOC

par Isabelle Breuil

$++++++++++++++++++++++++++++++++++++++++++++++++++++++$

5. INTÉGRATION DES FONCTIONNALITÉS D'OPENCAT AU CATALOGUE EN LIGNE DE LA BIBLIOTHÈQUE MUNICIPALE DE FRESNES par Pierre Bournerie 

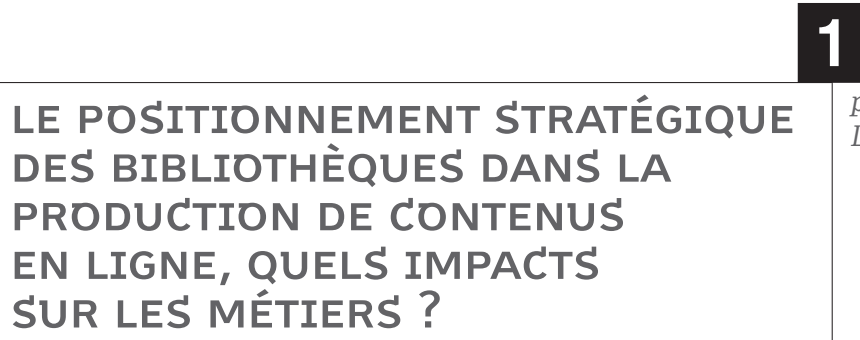

\section{LE BIBLIOTHÉCAIRE PRODUCTEUR DE CONTENUS ?}

\section{UN ACTEUR DE LA SOCIÉTÉ DE L'INFORMATION ET DU SAVOIR}

La dissémination générale d'outils facilitant l'accès et la transformation de l'information disponible sur le Web font que la technicité du bibliothécaire devient l'apanage de tous. Les professionnels de l'information et de la documentation doivent prendre acte de ces transformations, d'autant qu'ils subissent une dépréciation induite par le rôle prépondérant des moteurs de recherche et des réseaux sociaux dans l'accès au savoir. L'enjeu est d'être présent sur ces parcours informationnels empruntés par les publics. Les bibliothécaires ne peuvent plus se contenter de ne participer à la chaîne du savoir qu'en accumulant et en organisant les contenus documentaires disponibles. De même, ils ne peuvent plus limiter leur action de médiation, " traditionnelle » mais essentielle, de valorisation de l'offre documentaire à la simple présentation de nouveautés, ou aux sélections bibliographiques reproduisant les quatrièmes de couverture. Des formes plus abouties de productions peuvent être proposées, afin de transmettre aux usagers des contenus transversaux aux documents de la bibliothèque et à l'information du Web. Ce n'est donc pas sous le seul angle de la bibliothèque pour elle-même que doit être abordée la question de la production des contenus. Il est essentiel que celle-ci participe au mouvement global de la société de l'information et du savoir. Cette offre n'est en rien spontanée ou intuitive : elle est l'affirmation de choix qui reposent sur une politique éditoriale forte et cohérente avec le contexte de l'organisation, de ses collections, des publics et des territoires. 


\section{UN BIBLIOTHÉCAIRE JOURNALISTE ?}

Dans les actes de la conférence Enjeux politiques du document numérique ${ }^{1}$, Marc Bassoni écrit sur le journaliste : « Il trie, il "filtre", il hiérarchise les données et les arguments ; il "recommande" les résultats qui font consensus au sein des communautés expertes; il prend des précautions dans la reprise des arguments les plus controversés. En d'autres termes, il nourrit cette démocratie "réputationnelle" qui permet aux savoirs, moyennant une médiation efficace, de peser sur les débats publics et d'éclairer la marche du monde. »

De toute évidence, les enjeux liés à l'information et à la documentation et ceux du journalisme convergent. Ces métiers deviennent un art de la mise en perspective, de la construction d'une compréhension de l'information. Ils investissent le champ de la médiation et de la création de connaissances. Mais cette convergence ne signifie pas pour autant fusion. Le public comme les institutions n'attendent pas d'eux la même gestion de l'information au service des mêmes populations. Un bibliothécaire n'est pas un journaliste. Il est un agent public soumis à certaines restrictions quant à la publication des contenus. Il doit respecter le principe de neutralité du service public, son devoir de réserve et son obligation de discrétion professionnelle. Néanmoins, ces contraintes n'ont pas été conçues pour être une interdiction de la liberté d'opinion du bibliothécaire producteur. Celle-ci est d'ailleurs garantie par l'article 6 de la loi du 13 juillet 1983 portant droits et obligations des fonctionnaires ${ }^{2}$. Cette subjectivité est donc permise dans un cadre limitatif.

\section{LE BIBLIOTHÉCAIRE EN PREMIÈRE LIGNE ?}

Le bibliothécaire se pose comme un médiateur de confiance et est repéré comme tel au sein des communautés qui s'animent sur les espaces physiques et numériques. L'affirmation forte de cette identité professionnelle

1. Marc Bassoni, « Les pratiques documentaires des journalistes à l'heure des nouveaux médias : une rupture programmée », in Évelyne Broudoux et Ghislaine Chartron (dir.), Enjeux politiques du document numérique : actes de la troisième conférence. Document numérique et société, Paris, ADBS Éditions, 2010 (coll. Sciences et techniques de l'information).

2. Loi $n^{\circ}$ 83-634 du 13 juillet 1983. [En ligne] : < http://www.legifrance.gouv.fr/affichTexte.do?cidTe xte=LEGITEXT000006068812 >. 
peut se traduire par le passage d'une logique désincarnée à une logique incarnée de l'institution. À l'instar des bibliothécaires " geemiks »* de la médiathèque de la Skema Business School à Lille ${ }^{3}$ qui ont, chacune, décliné sur les médias sociaux une identité sur une discipline enseignée. Celles-ci sont d'abord repérées par les étudiants comme des personnesressources de confiance impliquées dans leur communauté avant d'être perçues comme bibliothécaires. Cette incarnation personnifiée peut se limiter à indiquer qui est le producteur du contenu. Elle peut être aussi fictive, à l'image de Miss Média à la bibliothèque municipale de Metz ${ }^{4}$. Cette expression "subjective » n'est pas toujours bien assumée par les bibliothécaires. L'ancrage profond d'une conception française de l'agent public qui doit s'effacer devant l'institution garante de neutralité, ainsi que plusieurs décennies de retrait derrière la collection, font qu'il y a encore une grande hésitation des professionnels à mettre en avant leur expertise sur les contenus. Rappelons que le bibliothécaire ne s'exprime pas selon ses convictions personnelles ou une quelconque pression des utilisateurs, il s'inscrit dans un projet de politique publique.

\section{PRODUIRE DES CONTENUS EN LIGNE : QUEL POSITIONNEMENT STRATÉGIQUE ?}

La présence durable en ligne d'une bibliothèque par ses contenus ne se réduit pas à une collection d'outils, ni à un acte ponctuel de communication. Celle-ci doit être en cohérence avec ses missions de service public liées à l'information, à la formation et à la culture et s'inscrire dans les projets de l'établissement. Un positionnement stratégique est donc à définir. Deux types de démarches nécessairement poreuses peuvent être adoptés. L'une est à dominante « institutionnelle », l'autre est à dominante " connaissance ». Le choix de l'une de ces deux dominantes participera à la définition des critères qui vont préciser cette présence en ligne.

3. < http://www.skema-bs.fr/campus/lille/>.

4. Marie-Paule Doncque, " Miss Média, nouvelle figure de Metz », in Jean-Marc Vidal (dir.), Faire connaître et valoriser sa bibliothèque : communiquer avec les publics, Villeurbanne, Presses de l'enssib, 2012 (coll. La Boîte à outils ; 27). 


\section{UNE APPROCHE « INSTITUTIONNELLE 》}

Cette approche s'adresse d'abord aux usagers fréquentant la bibliothèque, et de manière plus large à la communauté des habitants du territoire où se situe l'équipement. Les contenus arborent l'identité institutionnelle incarnée par le logo et le nom de la bibliothèque. L'information diffusée est proche d'une communication institutionnelle visant à faire connaître les informations pratiques, à promouvoir les services, la programmation culturelle ou encore les contenus produits comme les conseils de lecture, les bibliographies ou les dossiers thématiques.

Le site Web de la bibliothèque est certainement le canal le plus approprié pour positionner ces contenus. Mais ils peuvent aussi se décliner en " profils institutionnels "sur des médias sociaux tels que Facebook, Twitter ou encore sur des blogs.

Parce qu'évidente, cette identité numérique institutionnelle est la plus largement adoptée. Elle présente néanmoins l'inconvénient d'être peu lisible, hormis pour les affranchis, sur un Web dans lequel l'approche privilégiée est d'abord thématique et subjective, et où partager les mêmes centres d'intérêts prime sur l'institution ou la marque.

\section{UNE APPROCHE « CONNAISSANCE 》}

La démarche à dominante " connaissance » vise à participer à une communauté d'intérêts sur un thème au nom de la bibliothèque. La pertinence des contenus est donc essentielle. Cette identité thématique peut se décliner sur un blog, un portail à part entière indépendant du site institutionnel ou sous la forme d'une page Facebook ou d'un profil Twitter thématisés. L'image de la bibliothèque est portée sous l'angle des contenus proposés et complétée par l'affirmation subjective des bibliothécaires producteurs. Nous sommes ici dans une logique de médiation. Il est donc pertinent que ces contenus répondent à un besoin documentaire repéré ou supposé. Il ne s'agit pas simplement de répondre à une demande, mais de susciter la curiosité et engager le visiteur dans la découverte de nouvelles ressources et l'exploration d'un sujet. Il convient de souligner que la création de ce 
type de contenu sera d'autant facilitée si elle s'inscrit dans le cadre d'une politique documentaire ${ }^{5}$.

La cible est un public considéré de manière globale. Un amateur de musique peut aussi bien appartenir à la communauté des usagers de la bibliothèque, qu'à celle des habitants du territoire dans lequel s'inscrit l'équipement, ou encore à celle de la communauté des internautes " amateurs de » qui s'anime sur le Web social. Chacune de ces communautés pouvant s'intégrer aux autres, la bibliothèque se positionne dans toutes les sphères d'usagers possibles qu'elles soient matérielles ou numériques. Ainsi nous nous libérons d'une segmentation exclusivement " démographique » des publics. Il ne s'agit plus de produire des contenus pour les adolescents ou pour un public chômeur mais de s'adresser à des personnes qu'elles soient jeunes ou seniors, abonnés, habitants ou internautes.

Ces deux démarches ne sont pas exclusives. Elles peuvent être complémentaires voire imbriquées. Ce qui importe est d'assumer une démarche principale qui induira la cible visée et les types de contenus proposés.

\section{DES CONTENUS À LA RENCONTRE DES USAgeS}

Le choix des outils de production et de diffusion des contenus est indissociable de la réflexion sur le positionnement stratégique. Si ce choix influera sur le type de dispositif à déployer en interne, notamment sur la chaîne de validation et de publication des contenus, il affectera aussi le niveau de service offert à l'usager. La question des usages possibles est fondamentale car elle est la clé de l'appropriation des contenus proposés et de la dynamique de partage, voire de participation dans certains cas. Dans l'élaboration d'un dispositif de production de contenus, l'outil doit donc être en adéquation avec le besoin repéré et les usages constatés au sein de la communauté visée. En d'autres termes, l'outil choisi doit permettre d'inscrire les contenus dans les environnements informationnels numériques quotidiens du public visé.

5. Voir la contribution de Jérôme Pouchol : «Les dossiers documentaires numériques de la Médiathèque intercommunale Ouest Provence », p. 66. 


\section{ORGANISER LA PRODUCTION DE CONTENUS}

\section{LA PRODUCTION DE CONTENU EST UN PROJET}

Comme tout projet, la production de contenus implique la définition d'objectifs. La réflexion sur le positionnement stratégique devrait permettre de répondre aux questions qui préfigureront ce que sera la ligne éditoriale : Pourquoi ? Quoi ? Et pour qui ?

Il est important ensuite de faire un état des ressources humaines, matérielles et financières disponibles et celles nécessaires pour mener à bien son projet, ainsi que de déterminer les compétences mobilisables ou celles à acquérir afin de prévoir un plan de formation. Ces éléments vont déterminer le volume et la fréquence des publications, le type de contenus, le choix des outils de publication et de diffusion et, le cas échéant, élaborer un plan d'action d'accompagnement des bibliothécaires.

Ce travail peut être conduit par un groupe projet constitué de bibliothécaires volontaires, d'éventuels partenaires et de responsables de services de l'organisation concernée. Parce qu'il ne peut y avoir de production de contenus numériques sans infrastructure, le directeur du service informatique doit participer à cette réflexion afin qu'il appréhende les demandes techniques. Son regard est nécessaire dans le choix du logiciel de publication, son installation et ses évolutions possibles. Il en est de même pour le responsable du service communication afin de valider la présence Web de la bibliothèque via des services satellites du portail institutionnel, ou pour l'élaboration d'une charte graphique. Enfin, à défaut de sa participation, l'élu doit être tenu informé de l'avancée du projet. Sans volonté politique, un projet contiendra une part d'incertitude qui risque d'être dommageable, a fortiori si celui-ci demande des moyens pour être conduit à terme.

Tout projet implique aussi de réfléchir à une évaluation dès la phase de conception. Il convient d'en préciser ses objectifs et d'en déterminer les critères, au-delà des simples bilans chiffrés d'audience. Une analyse qualitative de ces chiffres s'impose : provenance géographique des visiteurs, mots-clés utilisés pour aboutir au site, parcours dans les contenus. Il convient aussi de mesurer : le niveau d'implication de l'équipe en regard des moyens déployés, le respect de la ligne éditoriale et des règles de 
publication, l'impact de cette production sur les services de la bibliothèque ou le constat d'une nouvelle notoriété au sein des communautés visées. Un projet de production de contenus doit être sans cesse réinterrogé afin d'être ajusté voire réadapté. Si des bilans étapes sont nécessaires, il est important d'inscrire ces projets dans la durée.

\section{ORGANISER LA CHAÎNNE ÉDITORIALE}

La chaîne éditoriale s'organise autour de trois dispositifs indissociables : une ligne éditoriale, une charte rédactionnelle et un circuit de production et de validation des contenus.

\section{une ligne éditoriale et une charte rédactionnelle}

La ligne éditoriale est directement tributaire des choix adoptés lors de la réflexion sur le positionnement stratégique et les moyens à engager dans la réalisation du projet ${ }^{6}$. Elle apporte une cohésion globale à l'ensemble des contenus produits. Pour aider les contributeurs, une charte rédactionnelle peut compléter cette ligne éditoriale. Cette charte fixe de manière concise les règles communes de rédaction, tout en ménageant un juste équilibre entre la nécessaire formalisation de l'écriture et la créativité des rédacteurs.

Ces documents de référence nécessitent des mises à jour régulières car les contextes évoluent en permanence.

\section{une chaîne de production et de validation (workflow*)}

Pour gérer efficacement d'un bout à l'autre la chaîne de production de contenus, il convient de formaliser les différentes étapes du circuit et le rôle tenu par chaque membre de l'équipe éditoriale. Ce processus ne doit pas être trop administratif au risque de diminuer la réactivité nécessaire à un média Web et de freiner l'implication des bibliothécaires. À titre indicatif, ces étapes peuvent se découper ainsi :

6. Ariane Gaudefroy, Une ligne éditoriale, à quoi ça sert ? [En ligne] : < http://redacteurweb.wordpress.com/2008/12/08/ligne-editoriale-definition/ >. 


\section{ENCADRÉ LE CIRCUIT DE PRODUCTION}

- le choix des thèmes à traiter inspiré de la ligne éditoriale ;

- identification des personnes ressources dans l'équipe : volontariat ou obligation? ;

- collecte des informations pour rédiger l'article ;

- la rédaction du contenu par le bibliothécaire rédacteur via l'outil de publication, en portant attention aux règles d'écriture Web ;
- le choix des illustrations multimédias ;

- le contrôle du respect de la charte rédactionnelle et validation du fond, de la forme, des aspects juridiques par le responsable de la publication ;

- la mise en ligne du contenu : par ce dernier ou directement par le rédacteur après validation?

Ce circuit demande la coordination d'un responsable de publication. Via l'interface d'administration de l'outil de publication ${ }^{7}$, celui-ci détient les droits d'administration pour la gestion des contenus et des droits des contributeurs. Il assure ainsi un contrôle qualité de ce qui est publié. Il peut refuser la publication d'un article si celui-ci n'est pas en conformité avec la ligne éditoriale ou la charte rédactionnelle. Il relance et accompagne l'équipe des rédacteurs et il veille à ce que l'investissement temps/ travail reste dans des proportions raisonnables. La légitimité de la personne qui assume ce rôle au sein de l'équipe est donc fondamentale.

\section{FAIRE DU BIBLIOTHÉCAIRE UN PRODUCTEUR DE CONTENU}

\section{QUeLles COMPÉTENCES ?}

La mobilisation des compétences nécessaires doit être menée en amont du projet. Ce sont de nouveaux savoir-faire qui viennent se greffer aux compétences traditionnelles du bibliothécaire. Véronique Mesguich indique $^{8}$ qu'ils sont de diverses natures :

7. Voir la contribution de Lionel Dujol : « Panorama des outils de production en ligne », p. 167.

8. Voir sa contribution: "Construire la médiation documentaire par les publics : les portails thématiques de l'Infothèque », in Xavier Galaup (dir.), Développer la médiation documentaire numérique, op.cit., pp. 92-103. 
- compétences techniques : maîtrise des outils de publication, de diffusion, de recherche et de veille sur Internet;

- compétences rédactionnelles : savoir produire des contenus, écrire pour le Web, réaliser des documents synthétiques ;

- compétences relationnelles : savoir maîtriser l'interactivité en ligne et plus particulièrement sur un média social ;

- compétences juridiques : connaissances en matière de droits d'auteurs, droit à l'image, maîtrise des licences libres Creative Commons...;

- Nous pouvons y ajouter des compétences plus stratégiques comme celles de la maîtrise du contexte numérique et la compréhension des enjeux.

\section{ACCOMPAGNER ET FORMER LES PERSONNELS}

Le bibliothécaire ne peut acquérir seul ces savoir-faire sans un plan d'accompagnement au sein de l'équipement, avec une reconnaissance de ces nouvelles compétences. La fonction de « bibliothécaire producteur de contenus » doit être inscrite dans les fiches de postes des agents concernés. Celles-ci indiquent les activités de production du professionnel ainsi que les compétences requises pour les exercer. Cette activité s'intègre au temps de travail réadapté en conséquence car elle ne peut s'ajouter aux activités déjà assumées. Cette révision des profils permet d'orienter un plan de formation professionnelle mis en œuvre par le service des ressources humaines.

Mais cela ne saurait justifier l'économie d'un plan d'accompagnement en intra vers une culture numérique commune et partagée. Cela peut être la mise en place d'ateliers pratiques en lien avec les outils à utiliser ou avec des usages numériques à investir, ou un cycle de conférences internes relatives aux enjeux du numérique dans la société du savoir et la place du bibliothécaire en son sein. Cette programmation a pour objectif d'apporter une " hauteur » nécessaire à la compréhension des projets.

La veille prend une dimension cruciale lorsqu'on demande au bibliothécaire de produire des contenus intermédiaires valorisant des ressources Web sur un thème donné. Mais insuffler une pratique de veille au sein 
d'une équipe est un travail au long cours, d'autant plus difficile si celleci est conduite de manière individuelle, sans une véritable coordination liée à un projet de production. La mise en place d'une veille collaborative en intra peut être aussi un outil d'accompagnement efficace et stratégique $^{9}$. Il garantit la diffusion d'un même niveau d'information à chacun, la construction d'une connaissance commune et une appropriation des outils de veille.

Enfin, une culture numérique se renforce par la pratique. Expérimenter sur son poste informatique professionnel de nouveaux outils est une activité indispensable. Sauf que bien souvent un accès bridé à Internet au sein des établissements, souvent pour des raisons légitimes de sécurité et de maintien de la bande passante, limite cette expérimentation. Nos exigences de service aux publics et d'adaptation professionnelle sont pourtant autant légitimes que les nécessités de sécurité, le dialogue avec la direction du service informatique dès la construction du projet s'avère ainsi impératif.

9. À l'instar du blog Face B des bibliothécaires de Romans-sur-Isère : < http:faceb.viabloga.com >. 


\section{2}

\section{CONTENUS DOCUMENTAIRES EN par LIGNE : UNE TYPOLOGIE Christelle \\ Di Pietro} EN 8 PRODUITS

Le service documentaire en ligne est un type de service spécifique en bibliothèque qui s'organise autour de trois composantes : fournir une prestation (en l'occurrence de nature documentaire) au moyen d'une organisation combinée à un outil, destinée à satisfaire les besoins d'information d'un public ${ }^{1}$. Ce service d'informations est un service à part entière. Comme les autres services, il doit être identifié et doté de moyens propres : une équipe coordinatrice, des objectifs, des moyens d'évaluation.

Ce service comporte trois fonctions essentielles :

- informer : fournir des données structurées et sélectionnées ;

- communiquer : transmettre, diffuser et valoriser ;

- médiatiser : alimenter, rendre accessible et gérer le contenu.

Le produit documentaire, prestation fournie par ce service, est un support d'information issu d'un traitement spécifique de l'information et réalisé à partir de documents primaires* et/ou secondaires*. Dans cette situation, le bibliothécaire n'est pas un créateur de contenus : il les médiatise. L'information ainsi transmise prend la forme d'un document secondaire ou tertiaire, conçu pour répondre à des besoins d'information, sous des formes diverses : bibliographie, bulletin bibliographique, bulletin de sommaires, dossier documentaire, dossier de presse, diffusion sélective d'information (DSI), revue de presse*, état de la question, synthèse documentaire...

1. Bertrand Calenge, Accueillir, orienter, informer : l'organisation des services aux publics dans les bibliothèques, Paris, Éditions du Cercle de la Librairie, 1996 (coll. Bibliothèques). 


\section{UNE TYPOLOGIE DES PRODUITS DOCUMENTAIRES}

On peut distinguer deux grandes familles de produits documentaires : les produits de signalement et les produits de synthèse. Après une typologie de sept de ces produits, nous détaillerons l'un d'entre eux : les brèves de l'enssib.

\section{PRODUITS DE SIGNALEMENT}

Connaissant avec le numérique un regain d'intérêt, ils sont les prestations de base attendues de tout service documentaire proposé par une bibliothèque. Citons : la revue de sommaires, le guide de recherche, la liste de nouveautés, l'agenda, le catalogue de périodiques ou d'ouvrages ou encore la revue de presse...

Ce sont des produits qui partent d'une demande et proposent des contenus basés sur l'exploitation, la valorisation des collections et la politique culturelle de la bibliothèque.

\section{La liste de nouveautés}

\section{Définition}

Souvent mensuelle, c'est une bibliographie non thématisée qui liste les dernières acquisitions de la bibliothèque. Elle peut être exhaustive ou ne comporter qu'une sélection. Il s'agit de la traditionnelle rubrique " coups de cœur du bibliothécaire "souvent présente sur les sites Web des bibliothèques. Elle sert d'étagère virtuelle, équivalent en ligne des présentoirs.

\section{Publics visés}

Le premier public visé est celui de la bibliothèque puisqu'il s'agit de valoriser les collections existantes et d'inciter à leur utilisation.

\section{Intérêts et limites}

Cela permet d'informer de l'activité de la bibliothèque, en valorisant le rôle de filtre et de recommandation du bibliothécaire.

Cependant, l'approche simplement descriptive demeure souvent favorisée au détriment d'une approche critique. Étrangement, le champ de la critique littéraire est peu investi par le bibliothécaire qui serait pourtant pertinent et légitime. 
Mise en ouvre

Le blog est souvent l'outil privilégié pour ces contenus. Il permet une autonomie de rédaction tout en étant accessible depuis le site de la bibliothèque. Le blog permet aussi d'avoir une identité propre avec sa charte rédactionnelle et d'introduire une personnalisation (identité numérique) du bibliothécaire : c'est le cas par exemple d'Everytouthèque, le blog des critiques des bibliothécaires de
Valence-Romans ${ }^{2}$ qui s'expriment à la première personne. Comme exemple de blog critique, et de surcroît co-construit avec les usagers, on peut également retenir celui des bibliothèques de Brest ${ }^{3}$. Il fonctionne avec un comité de lecture mixte (parents, bibliothécaires, enseignants) aux avis quelquefois (voire souvent!) divergents. Les avis sont commentés par des élèves de CM2 qui viennent les conforter ou les contredire.

2. < http://everitoutheque.viabloga.com >.

3. « On lit trop dans cette bibli... » : < http:// on-lit-trop.fr >.

\section{La revue de sommaires}

\section{Définition}

C'est une compilation généralement thématique, souvent hebdomadaire ou mensuelle, des sommaires de plusieurs publications périodiques. Dans l'idéal, c'est une base de données dans laquelle on peut naviguer par titre de périodique ou par sujet et s'y abonner soit par courrier électronique, soit par fil RSS. Elle peut aussi être un simple bulletin téléchargeable en format PDF.

\section{Publics visés}

C'est un produit qui s'adresse à un public académique qui a besoin de faire une veille d'actualité sur son champ.

\section{Intérêts et limites}

C'est une valorisation simple et peu coûteuse des abonnements qui sont, eux, onéreux, en particulier ceux qui concernent des publications académiques.

Le sommaire n'est pas protégé par le droit d'auteur tant qu'il n'est pas 
reproduit dans son exacte forme d'édition originale, cela en fait un contenu tiers facile à exploiter. Du coup, l'exploitation demande des moyens humains importants pour ne pas justement reproduire la forme originale. La mise à jour est cruciale, une fois débutée, la revue de sommaires doit être consciencieusement alimentée, titre à titre.

\section{Mise en ouvre}

La seule contrainte est la nécessité de ressaisir des informations (soit manuellement, soit en numérisant et océrisant) puisque la forme originale ne peut être utilisée. Afin d'optimiser la recherche, il faut prévoir un outil de type base de données (facile à créer avec des Content Management System*, CMS, comme Drupal) avec quelques champs obligatoires : titre du périodique, auteur/éditeur, ISSN, thématique/sujet.

Les niveaux de complexité des outils sont très variables d'un établissement à l'autre.
Si certaines revues ont choisi de simplement publier des bulletins périodiques avec des options de filtre (voir, par exemple, celle de la cellule juridique de la $\mathrm{CFDT}^{4}$ ), d'autres proposent des syndications de fils RSS, sans reprise des données, comme, par exemple, le portail Netvibes du centre de documentation de la Délégation générale à l'Outre-Mer (DéGéOM) 5 .

Plus complexe est la mise en relation de la base de sommaires avec le catalogue ou avec les textes intégraux des articles qui nécessite un traitement des informations avec des formats normalisés. C'est le cas de Sign@l, la base de sommaires collaborative éditée par Sciences Po Lyon) ${ }^{6}$.

4. < http://www.cfdt.fr/portail/le-carnet-juridique/revue-de-sommaires-asp_5063 >.

5. < http://www.netvibes.com/revue-sommaires $>$.

6. < http://doc.sciencespo-lyon.fr/Signal/ >. Voir la contribution de Carole Tilbian : «Valoriser les périodiques en SHS : le modèle collaboratif de Sign@l », p. 40.

\section{Le guide de recherche documentaire}

\section{Définition}

Il permet de former à la recherche d'informations, à travers des parcours de formation thématisés ou en explicitant la méthode de recherche documentaire. 
Les premiers guides de recherche documentaire ont été conçus pour les étudiants, c'est le cas du célèbre Conseil aux étudiants en recherche d'informations efficace (CERISE) ${ }^{7}$, lancé en 1996 et intégralement refondu en septembre 2013.

Plus tard sont apparus les guides destinés à des publics élargis, comme le Guide de la recherche en bibliothèque (Grebib) de la $\mathrm{BnF}^{8}$. La dernière génération de guides s'est construite autour de la notion de «modules-focus » sur des points précis, comme Méthodoc, conçu par l'université de Rennes $2^{9}$.

\section{Publics visés}

Le guide s'adresse à tous ceux qui ont des besoins réguliers d'informations structurées, particulièrement les étudiants et publics scolaires, et destinées à être restituées, souvent sous forme académique. Il permet de systématiser une démarche sans avoir pour autant des connaissances approfondies ou professionnelles en matière de recherche documentaire : comment chercher tel type d'information? Comment utiliser tel outil

7. < http://urfist.enc.sorbonne.fr/cerise/ >.

8. < http://grebib.bnf.fr >.

9. < http://methodoc.univ-rennes2.fr >. documentaire ? Comment trouver des informations sur Internet?

Il est en revanche souvent peu adapté aux besoins ponctuels d'information du grand public.

\section{Intérêts et limites}

Conçu comme une aide à l'autonomie de l'usager, il met en relation des besoins de recherche avec la compétence qu'ils exigent. Il doit cependant être élaboré en regard de besoins exprimés, et être testé pendant son élaboration auprès de son public pour vérifier sa pertinence. Il ne prend pas obligatoirement en compte les nécessités de ceux qui ont un besoin d'information ponctuel et précis, ne souhaitant pas faire l'apprentissage d'une méthode de recherche.

\section{Mise en ouvre}

Le guide peut prendre l'aspect d'une fiche pratique, d'un produit sur un site Web, d'un parcours de recherche, de listes d'outils. La qualité de la réflexion sur le parcours cognitif mis en œuvre par les publics auxquels on s'adresse est primordiale. Il s'agit de partir de la réflexion de l'usager plutôt intéressé par une recherche thématisée, comme la recherche de sources dans un domaine, et de développements techniques précis 
(mode d'emploi d'une base de données par exemple). L'enquête de besoins s'avère nécessaire, de même que la co-construction $\mathrm{du}$ guide avec ses usagers. De ce point de vue, celui réalisé par
Isabelle Breuil avec Arbradoc ${ }^{10}$ pour l'université de Paris 8 est exemplaire ${ }^{11}$.

10. < http://arbradoc.bu.univ-paris8.fr >.

11. Voir sa contribution : "Concevoir une plate-forme d'autoformation à la recherche documentaire : Arbradoc », p. 48.

\section{L'agenda}

\section{Définition}

C'est un calendrier d'événements thématiques portant sur l'activité d'un établissement ou sur la surveillance exhaustive d'un domaine d'activité.

\section{Publics visés}

Variables : le public local ou l'intégralité d'un public intéressé à une thématique. L'un des objectifs est souvent de communiquer sur l'activité culturelle ou événementielle d'un établissement afin d'y faire participer son public.

\section{Intérêts et limites}

Outil de communication indispensable pour l'établissement, il est vivant et dynamique quand il est étendu à une thématique. Il permet de façon transversale de faire une veille active sur une thématique, mais l'alimentation peut vite se montrer fastidieuse, elle doit être quasiment quotidienne et tendre à l'exhaustivité pour être crédible.

\section{Mise en ouvre}

Il nécessite de mettre en place un dispositif de veille collaboratif pour systématiser la collecte des informations. Un répertoire de sources, avec un outil de partage de liens, régulièrement mis à jour peut être amplement suffisant.

Techniquement, il faut apporter un soin particulier à la recherche et aux options de filtres/tri : par date, par type d'événement, par sujet, et donc prévoir des catégories. On peut envisager une collaboration entre agendas en utilisant le 
format d'échange normalisé iCal, comme elle a existé entre l'agenda de l'enssib ${ }^{12}$ et Calendoc, agenda collaboratif qui était disponible sur

12. < http://www.enssib.fr/services-et-ressources/agenda-des-manifestations-professionnelles-et-scientifiques $>$.
Google Calendar. Le fil iCal permet aussi de s'abonner facilement et de récupérer automatiquement les informations sur son propre calendrier.

Il faut aussi envisager la possibilité de soumissions d'événements par le public avec validation.

\section{3 produits de synthèse}

Ce sont les produits qui nécessitent une rédaction originale par le bibliothécaire, afin de synthétiser, filtrer, ou vulgariser des informations. Ils sont par nature dynamiques et susceptibles d'être réactualisés. Citons : les bibliographies, les signets, les synthèses bibliographiques, les panoramas de presse, les dossiers documentaires... On trouve aussi dans cette catégorie les produits interactifs : sites Web, portails, blogs, wikis, réseaux sociaux ; et une catégorie particulière, les produits personnalisés ou produits à la demande : services de questions-réponses, synthèse documentaire, diffusion sélective de l'information, produits de veille. En bibliothèque, ces prestations, bien que générant une valeur ajoutée importante, sont généralement gratuites. On peut toutefois signaler le service de veille documentaire de la Bibliothèque nationale suisse qui pratique une approche payante par prestation ${ }^{13}$.

\section{La base de signets (ou bibliothèque de liens)}

\section{Définition}

Liste de sites Web, généralement classés par thématique, qui constitue un répertoire généraliste ou spécialisé. Chaque site peut faire l'objet d'une approche critique ou d'une description.

13. Voir leur offre en ligne : < http://www. nb.admin.ch/dienstleistungen/swissinfodesk/00769/index.html?lang=fr >. 


\section{Publics visés}

Les publics visés sont peu familiers de la recherche sur Internet ou souhaitent faire une recherche rapide sur une thématique précise. Aujourd'hui, ces répertoires sont davantage constitués pour partager en interne des ressources dans le cadre d'une veille collaborative, à l'exception notable des signets de la $\mathrm{BnF}^{14}$ dont l'importance de la base en fait un outil universel aussi bien adapté au grand public qu'à des spécialistes.

\section{Intérêts et limites}

Le principal intérêt est de proposer des ressources validées et fiables dans un parcours de recherche documentaire. Cela permet aussi à la bibliothèque de participer à sa mission patrimoniale dans un domaine précis, comme l'a fait le département Rhône-Alpes de la bibliothèque de Lyon avec son recensement de sites sur les élections régionales en 2010-2017. Lorsqu'il n'est pas constitué à des fins patrimoniales, les mises à jour du répertoire posent problème. Il n'y a guère que la $\mathrm{BnF}$ qui puisse encore continuer à entretenir sa base (elle est maintenue de façon transversale

14. <http://signets.bnf.fr >. par les agents producteurs de contenus en ligne de la $\mathrm{BnF})^{15}$, bien que le nombre de visite ait été divisé par 6 entre 2006 et 2012.

\section{Mise en œuvre}

Encore présents sur les sites de bibliothèques malgré une certaine désuétude ${ }^{16}$, les répertoires de signets prennent la forme d'une ressource spécialisée sur un thème. Ils sont aujourd'hui relancés par l'archivage du Web et surtout la veille collaborative.

Quelques pistes à creuser :

- sur quels critères choisir les sites ? Quelle grille d'analyse?

- selon quel classement? Arborescence, classification, thématiques...

- que présenter ? Résumé, commentaire critique, explication des critères de sélection...

15. Sur l'organisation interne des signets de la BnF : voir Marie-Françoise Garion, Bibliothèques de liens sur Internet et recherche en sciences humaines et sociales : la création d'une maquette pour un groupe de recherche international, Mémoire d'étude de conservateur des bibliothèques, sous la direction de Christian Jacob : Villeurbanne, enssib, 2005. [En ligne] : < http://www.enssib.fr/ bibliotheque-numerique/document-630 >.

16. Voir la page de Bibliopédia qui en recense quelques-uns, toutes bibliothèques confondues : < http://www.bibliopedia.fr/index php/Répertoires_de_signets >. 
- quelle veille et quel maintien des sites?
- quel choix de collaboration avec les internautes en matière d'alimentation?

\section{La bibliographie}

\section{Définition}

Liste structurée de références bibliographiques, elle peut être signalétique (par titre, auteur) ou analytique (avec un plan thématique), limitée dans le temps, sélective ou exhaustive.

\section{Publics visés}

Souvent réalisée comme produit d'accompagnement d'un événement (exposition, conférence) ou d'un apprentissage (cours universitaires, préparation aux concours), la bibliographie permet d'approfondir un sujet. Elle peut aussi être conçue comme un produit autonome réalisé à la demande d'un usager - rejoignant par là le service de référence, comme par exemple le service bibliographique du Centre de documentation Économie-Finances ${ }^{17}$, ou dans le cadre d'un service spécialisé ${ }^{18}$.

17. < http://www.economie.gouv.fr/cedef/recherches-et-veilles-bibliographiques $>$.

18. Comme celui de la BnF : < http://www.bnf.

\section{Intérêts et limites}

L'intérêt peut être de proposer un produit sur mesure à son usager ou de valoriser ses collections le temps d'une exposition.

Les limites sont celles de l'obsolescence, ce sont par définition des produits qui se périment vite, selon les thématiques abordées.

\section{Mise en œuvre}

Penser à tenir compte du niveau de public visé et dater la bibliographie, choisir un format: HTML ou PDF téléchargeable, voire XML. Certaines bibliothèques ont fait des choix plus créatifs, axés sur la sérendipité et la découverte, comme les biblio-livres de la $\mathrm{Bi}$ bliothèque départementale de prêt (BDP) de l'Ardèche ${ }^{19}$, réalisés avec Calaméo, feuilletables en ligne et téléchargeables ; ou bien celles des

$\mathrm{fr} / \mathrm{fr} /$ collections_et_services/bibliographies. html $>$.

19. < http://bdp.ardeche.fr/EXPLOITATION/Default/thematique.aspx $>$. 
bibliothèques de Genève, réalisées par des graphistes avec Issuu ${ }^{20}$. Il est préférable de choisir un format normalisé de rédaction des notices afin d'harmoniser la présentation. Le plus simple est de choisir la norme ISO 690 (Z 44-005) ou un format simplifié, comme le propose

20. < https://www.ville-ge.ch/bm/fr/savoirs/ selection_thematique_choix.php. > le guide de rédaction de la $\mathrm{BU}$ de l'université de Reims ${ }^{21}$.

Enfin, il faut déterminer à l'avance la politique d'archivage, de désherbage ou de mise à jour.

21. Catherine Miconnet, Agnès Faller, Bibliographie et références bibliographiques, SCD de l'université de Reims Champagne-Ardenne, janvier 2012. [En ligne] : < http:// www.univ-reims.fr/site/bibliotheques/memoires-et-theses/gallery files/site/1/1697/2 0119/20141/20145/33422.pdf >.

\section{La synthèse documentaire}

\section{Définition}

C'est un produit, souvent multimédia, qui nécessite la constitution d'un corpus d'informations synthétisées de façon à constituer un ensemble cohérent. Il traite souvent de l'actualité d'un sujet, ou de l'état d'une recherche à un moment donné (on parle alors "d'état de l'art »).

La synthèse fait souvent partie d'un dossier documentaire thématique.

\section{Publics visés}

Le public visé s'intéresse à un sujet particulier qu'il souhaite approfondir, pour des raisons personnelles ou pour un travail scolaire. La synthèse est réalisée à partir d'une demande précise ou d'un sujet d'actualité. C'est le cas des dossiers de veille réalisés par le service Veille et analyses de l'Institut français de l'éducation (IFÉ, ex-INRP) qui s'empare d'un sujet, en fait une analyse et une synthèse approfondie ${ }^{22}$.

22. < http://ife.ens-lyon.fr/vst/DA/ListeDossiers.php >. 


\section{Intérêts et limites}

Il s'agit d'un produit unique à forte valeur ajoutée, il a donc un public potentiel important, à condition que le référencement de la rubrique Web soit soigneusement effectué ${ }^{23}$. Il nécessite un travail de recherche important qui peut être conduit sur plusieurs mois et des compétences indiscutables en matière d'exploitation de l'information et de synthèse. Sa durée de vie est par définition courte : portant sur l'actualité d'un sujet, il se périme vite.

\section{Mise en ouvre}

La dimension rédactionnelle est importante : il ne s'agit pas seulement de compiler des références mais de les synthétiser selon un

23. Voir la contribution d'Isabelle Rouquet : «Écrire pour le Web », p. 155. plan ordonné et problématisé. Ces produits nécessitent la mise en place d'un circuit rédactionnel structuré, avec un responsable de rédaction chargé de la validation et une charte éditoriale précise.

Il faut aussi prévoir une alimentation régulière afin de constituer un corpus vivant qui suscite une fréquentation assidue. La question de la réactualisation/archivage est importante. Dans le cas de l'IFÉ, le choix a été d'archiver les dossiers de 2004 à 2008 et non de les réactualiser. La réactualisation s'avère d'ailleurs souvent plus complexe à mettre en œuvre qu'une refonte complète d'un dossier.

D'un point de vue technique, la synthèse prend souvent la forme d'un dossier pdf à télécharger, l'idée est de proposer un produit indépendant de son support.

\section{PRODUIT DE VEILLE EN FORMAT BLOG : L'EXEMPLE DES BRÈVES DE L'ENSSIB}

La fin de Biblio.fr en 2009 a entraîné l'arrêt de la diffusion d'informations nécessaires à la communauté professionnelle. C'est dans ce contexte que l'enssib a développé ses services de veille pour les professionnels ${ }^{24}$ dont les trois premiers - l'agenda des manifestations scientifiques, les offres d'emploi et la veille sur l'actualité - ont ouvert début 2010. Pour ce faire, l'école s'est appuyée sur une cellule de veille composée d'une bibliothécaire et d'un webmestre, au sein du service Diffusion des savoirs. 


\section{DÉFINITION D'UNE POLITIQUE ÉDITORIALE}

La charte de publication d'origine comportait cinq points essentiels :

- une veille en ligne publiée par et pour des professionnels des bibliothèques, de la documentation et des archives ;

- à partir de courts extraits d'articles « sourçables » en ligne ;

- des rédacteurs identifiés (cinq au début du projet) coordonnés par une responsable de rédaction ;

- des informations innovantes, factuelles, avérées ;

- des compléments documentaires sur le sujet abordé.

L'angle choisi est une veille " de niche » qui s'éloigne des informations dupliquées sur les autres blogs de la « biblioblogosphère », et portant sur des innovations appliquées ou des projets opérationnels. Les informations strictement techniques, ne présentant pas d'intérêt applicatif sont exclues, ainsi que les annonces de projets à venir plus ou moins lointains dans le temps. Chaque brève est complétée par des documents publiés dans la bibliothèque numérique de l'enssib, ils offrent un prolongement de réflexion ou des éclaircissements sur le sujet abordé.

Techniquement, c'est le module blog de Drupal, déjà utilisé par le site Web de l'enssib, qui a servi.

\section{ALIMENTATION ET VEILLE}

Un portail Netvibes a été élaboré afin de partager des ressources de veille communes aux rédacteurs pour alimenter le blog ${ }^{25}$. Des abonnements systématiques ont été souscrits à toutes les listes de diffusion et newsletter, gratuites ou payantes, des professionnels de l'info-doc (EBSI-L, Swisslib, ADBS, newsletter du réseau territorial de documentation, Maires-info, GFFI et dépêches AEF...). Les bases de données de presse (Factiva) sont aussi utilisées et le réseau relationnel a été mis à profit. 


\section{ENCADRÉ LA CHARTE ÉDITORIALE DES BRÈVES DE L'ENSSIB}

- un ton neutre et factuel ;

- une ou deux brèves par jour, dix lignes maximum ;

- un copié-collé d'un extrait d'article, ou une synthèse, ou une rapide traduction pour les articles en langue étrangère ;

- un titre propre ;

- des sources citées avec lien direct;

- des illustrations avec des images libres de droit ;
- un taguage « libre » avec harmonisation des tags ;

- un classement thématique en deux grandes catégories : monde de l'information et monde des bibliothèques, elles-mêmes subdivisées en sous-thèmes.

Ces règles ont évolué depuis : la longueur du texte a été allongée, d'autres sujets ont été ajoutés.

\section{UNE OFFRE DOCUMENTAIRE COHÉRENTE}

$++++++++++++++++++++++++++++++++++++++++++++++++++++++++++++++++++$

Le développement des services de productions de contenus doit être conçu comme une offre globale. Il serait inutile, voire néfaste, de cibler un ou deux produits de façon isolée sans concevoir une offre de service cohérente qui entre dans le projet scientifique et culturel de la bibliothèque. Chacun des produits proposés ici doit donc être envisagé comme une brique possible d'un ensemble construit autour de l'offre documentaire. 


\section{SCHÉMA STRUCTURATION D'UNE BRÈVE}

titre propre, différent de celui de l'article, avec une accroche

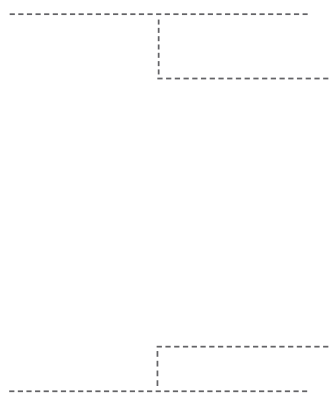

source de l'article: titre, nom du journal, date, avec un lien sur

l'article original

ressources enssib en lien avec le sujet abordé

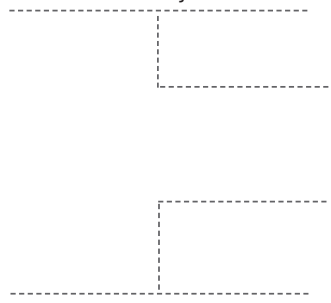

article raccroché à une catégorie / sous-catégorie chaque brève est structurée de façon identique.

\section{NOURRITURE CONTRE PÉNALITÉS}

Par Christelle DI PIETRO, le 25 Mars 2010

La bibliothèque universitaire du Western Kentucky accepte de la nourriture en paiement des pénalités de retard des étudiants. L'automne dernier, la bibliothèque a collecté plus de trois cents produits alimentaires non périssables qui ont été donnés aux oeuvres de l'église locale. La règle d'échange est simple : 1 prodnit $=1 \$$ remboursé. Nombreuses sont les bibliothèques américaines (et plus largement anglo-saxonnes) qui participent au programme Food for Fines : les bibliothèques publiques de Phoenix, celles de l'mininois, ou encore celles du comté de Jefferson.

Lire la suite : Food Accepted in Place of Library Fines (vidéo), WBKO channel, 23 mars 2010 et Pay Off Library Fines by Donating Food, myFOXphoenix, 22 mars 2010.

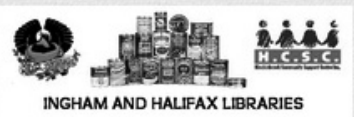

INGHAM AND HALIFAX LIBRARIES

\section{Food for Fine\$}

MONDAY 25TH MAY TO SATURDAY BTH JUNE

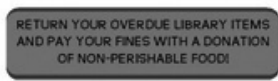

One can of food $=\$ 2$ fines to help those in need Up to a maximum of $\$ 20$ per borrower.
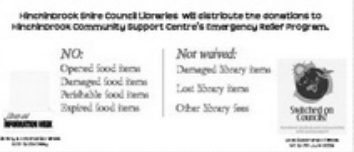

Affiche du programme Food for Fines des bibliothèques du comté d'Hinchinbrook en Australie

Pour aller plus loin :

- Rappels et pénalités, quelles pratiques ? Questions-Réponse $n^{\circ} 190719$, enssib 23 novembre 2009

- Qnelles règles pour les usagers en retard ? Questions-Réponse $n^{\circ} 18263_{2}$, enssib 23 juin 2009.

Catégorie : Le monde des bibliothèques, $\underline{\text { Rôle et fonctions des bibliothèques }}$ Services aux publics

Tags : Charité, États-Unis image d'illustration avec source et lien

Source : Christelle Di Pietro, " Nourriture contre pénalités », Brèves de l'enssib, 25 mars 2010. [En ligne] : < http://www.enssib.fr/en/breves/2010/03/25/nourriture-contre-penalites >. 


\section{3}

VALORISER LES PÉRIODIQUES

EN SHS : LE MODĖLE

COLLABORATIF DE SIGN@L

\section{UNE TRADITION DOCUMENTAIRE REVISITÉE}

Conçu dans le but de valoriser les contenus de périodiques en sciences humaines et sociales (SHS), Sign@l décrit et donne accès à des unités documentaires de trois niveaux : des sommaires de revues ; une sélection d'articles issus de ces revues, indexés voire résumés ; une présentation sommaire des revues.

En mars 2014, Sign@l propose plus de 17000 sommaires issus de 280 revues différentes et référence plus de 70000 articles indexés issus de 72 revues.

Douze établissements, bibliothèques ou centres de documentation de l'enseignement supérieur majoritairement, ainsi que deux des principaux portails de revues en SHS, participent à l'enrichissement de la base.

\section{SIGN@L, UNE BASE RÉCENTE, UN LONG PASSÉ : 1983-2011}

Officiellement lancé en 2011, Sign@l est le fruit de vingt années d'efforts pour développer la visibilité et l'usage des collections de périodiques en SHS. Conscients que les revues scientifiques sont un des piliers des collections universitaires en SHS, les documentalistes de l'Institut d'études politiques (IEP) de Lyon ont multiplié les initiatives pour aider leurs usagers à exploiter efficacement le contenu de ces ressources. Contrairement à ce qui se pratiquait souvent dans le milieu universitaire, Sciences Po Lyon a toujours prêté ses revues imprimées. Mais comme elles n'étaient pas en libre accès, un travail de signalement et de dépouillement a également été développé.

L'aventure Sign@l remonte ainsi à 1983, année de création d'un Fichier de références bibliographiques d'informations politiques, économiques et sociales, intitulé Fripes. Produit documentaire assez traditionnel, ce 
fichier proposait des notices bibliographiques d'articles de revues en SHS (une vingtaine pour commencer), enrichies d'une indexation sujet mais également d'un résumé. Différents acteurs documentaires de la région Rhône-Alpes participaient initialement à l'alimentation de ce fichier que l'IEP de Lyon finira par maintenir et enrichir seul jusqu'à la fin des années 2000. Fripes contient alors les références de plus de 65000 articles, issus de 93 revues.

En 1998, les documentalistes de l'IEP constatent que les périodiques qui ne sont pas dépouillés sont nettement moins empruntés que ceux qui le sont. Face à l'impossibilité d'inclure les quelque 300 titres de la collection dans Fripes, ils décident de créer une base de sommaires, Somrev. Pendant quelques années, Sciences Po Lyon saisit les sommaires de revues dans une base accessible sur Internet, à la disposition de l'ensemble de la communauté universitaire. En 2005, Somrev passe en mode collaboratif. Entre 1998 et 2008, cette seconde base grossit jusqu'à proposer près de 98000 sommaires issus de 111 revues différentes.

Après dix ans de coexistence, compte tenu de la convergence du travail conduit dans l'une et l'autre base, Fripes et Somrev fusionnent.

\section{SIGN@L, UN MODĖLE COLLABORATIF DURABLE}

$++++++++++++++++++++++++++++++++++++++++++++++++++++++++++++++++++$

L'une des originalités notables de Sign@l tient à sa dimension collaborative. Le modèle de mutualisation remonte à Fripes. En 1983, les veilles partagées à l'échelle locale et impliquant des partenaires institutionnels aussi variés que la Chambre de commerce et d'industrie de Lyon, la Bibliothèque municipale de Lyon, le conseil général, les services de la Préfecture et des structures universitaires sont assez rares...

Si l'IEP de Lyon met en place Somrev seul, il voit les premiers partenaires le rejoindre assez spontanément : utilisateurs de la base, ils proposent de participer à son enrichissement. 


\section{STRUCTURATION DU RÉSEAU DE PARTENAIRES}

Le développement du réseau de partenaires répond à une double logique, à la fois géographique et institutionnelle. La proximité géographique constitue un atout majeur : sur les douze partenaires actuels de Sign@l, six sont des structures rhônalpines. La forte représentation des différents instituts d'études politiques de province au sein du réseau s'explique par la convergence des cursus et des abonnements souscrits.

L'organisation d'une journée d'étude sur le signalement de contenus de périodiques à Lyon en 2008 a par ailleurs eu un effet sensible sur l'accroissement du nombre de partenaires (5 nouveaux partenaires dans l'année qui suit).

Les partenariats restent toutefois informels puisque contrairement à ce qui sera décidé pour d'autres projets, aucune convention ne lie les acteurs de Sign@l. Chaque partenaire a le choix du corpus sur lequel il travaille. Si Sciences Po Lyon reste le pilote unique de la base et de ses développements, c'est bien le réseau qui est valorisé dans les actions de sensibilisation au projet. Cette dimension partenariale lui confère une légitimité plus grande, notamment lorsqu'il s'agit de demander des subventions. Il faut souligner que Somrev puis Sign@l ont toujours bénéficié d'un soutien marqué de la Région Rhône-Alpes grâce à des subventions consacrées aux aspects techniques du projet (serveurs, maintenance, développements informatiques).

\section{LE MODĖLE COLLABORATIF : QUELLE ORGANISATION ET QUELLE INFRASTRUCTURE ?}

Sign@l a été conçu comme un outil de production collaborative. La base doit permettre aux partenaires de saisir directement les sommaires de " leurs » revues sur le serveur de production. Sciences Po Lyon assure la coordination et le pilotage du projet et prend à sa charge :

- les coûts des développements informatiques : l'hébergement, l'administration et la maintenance de l'infrastructure informatique ;

- la gestion des utilisateurs et de leurs droits ; 
- la définition des règles de saisie et le contrôle des données ;

- la formation et l'accompagnement des partenaires ;

- l'indexation des articles.

Pour permettre au réseau de partenaires de communiquer facilement, une liste de diffusion a été mise en place.

Travailler en collaboration suppose enfin de partager des outils communs. Le développement des partenariats a ainsi été à l'origine d'importantes évolutions dans la structure même de la base et dans les modes de production proposés.

\section{L'ÉVOLUTION DES OUTILS, L'ÉVOLUTION DES MÉTIERS}

Sign@l est intéressant en tant que produit mais également en tant que projet inscrit dans la durée. Il témoigne des importantes mutations qui ont touché les métiers de la documentation et des bibliothèques depuis le début des années 1980.

L'histoire de Sign@l brasse tous les formats de collecte, de diffusion, de partage, de structuration des données bibliographiques, qui se sont succédé en trente ans. Fripes commence avec des notices bibliographiques au format papier, assez vite diffusées par Minitel et dès 1993 sur Internet. Fripes, Somrev puis Sign@l, marquent le passage d'un modèle de données plutôt simple à l'intégration de nouvelles normes de structuration, d'échange et de diffusion : mise en place d'un schéma d'échange en XML, création d'une interface php/MySQL, interopérabilité avec un moissonneur OAI-PMH...

La mise en place de Sign@l a donc supposé une veille active et une appropriation rapide des évolutions techniques notamment. La présence de documentalistes au profil informatique documentaire marqué au sein de l'IEP de Lyon a contribué au développement de ces bases. Le recours à un prestataire informatique extérieur reste toutefois nécessaire. 


\section{ÉVOLUTION DES OUTILS, IMPACT SUR LES CONTENUS}

Les évolutions opérées dans la structuration des données ont eu des répercussions directes sur le contenu même de la base.

En effet, la mise en place d'imports automatisés via l'OAI-PMH a rendu possible l'intégration en masse des données issues des portails en SHS (un premier partenariat avec Persée dès 2007 puis avec Cairn en 2009). Ce moissonnage OAI s'est traduit par un accroissement rapide du nombre de titres couverts mais il a également permis d'accéder à l'antériorité des sommaires de plusieurs revues. En 2014, les deux portails fournissent les sommaires de $25 \%$ de l'ensemble des revues.

De la même façon, la mise en place du schéma d'échange XML autorise des échanges de données avec d'autres structures productrices (base de sommaires de l'ENS-LSH en 2006).

La récupération automatique a également eu un impact sur la nature du travail documentaire ; deux modes d'enrichissement de la base coexistent donc : saisie manuelle traditionnelle ou import que le professionnel doit contrôler et dont il doit corriger les résultats. Sciences Po Lyon assure seul cette tâche de contrôle qualité.

\section{SIGN@L, DIFFUSION ET ACCÈS}

Depuis l'origine, l'objectif du travail fourni par le service documentaire de Sciences Po Lyon, qu'il s'agisse de dépouillement ou de signalement, a été de valoriser auprès du public le contenu des périodiques. Les données produites dans Somrev, Fripes, puis Sign@l ont toujours été diffusées le plus largement possible, via une interface Web librement consultable par tous. Le fichier Fripes avait été accessible sur le Serveur universitaire national de l'information scientifique et technique (SUNIST) et sur Minitel !

Les développements apportés au fil des ans visent tous à rendre la recherche plus facile mais également plus efficace, en essayant notamment de donner non seulement accès à la référence mais également au document primaire. 


\section{UNE PRISE EN MAIN AISÉE}

Parmi les fonctionnalités ajoutées dans l'interface de consultation, l'une concerne la recherche " sujet ». Nous savons tous que l'utilisation d'un langage structuré pour l'indexation peut poser problème auprès d'un public non initié (la majorité du public donc). Une fonctionnalité de complétion automatique a été développée : la saisie de quelques lettres suffit à faire apparaître dans un menu déroulant les mots du thésaurus comprenant cette chaîne de caractères.

Par ailleurs, afin d'optimiser la recherche bibliographique, Sign@l est devenu compatible avec le logiciel libre de gestion de références bibliographiques Zotero dès 2011.

Enfin, les utilisateurs peuvent suivre l'enrichissement de la base en s'abonnant aux flux RSS.

Les évolutions techniques visent également à permettre aux partenaires de personnaliser les interfaces de production et de consultation. Depuis 2011, les partenaires peuvent ajouter sur la page d'une revue un lien direct vers la notice de la revue dans le catalogue local ; chaque partenaire peut en outre indiquer quelles revues il détient, permettant ainsi à son public de limiter ses recherches avec ce critère.

\section{L'ACCÈS AU DOCUMENT PRIMAIRE}

L'un des enjeux des dernières années a été d'enrichir les notices bibliographiques traditionnelles en fournissant dès que possible des données d'accès : accès au texte intégral libre ou restreint ; lien vers Mir@bel (voir infra) pour chaque notice de revue. Il faut noter que dans $36 \%$ des cas, Sign@l permet un rebond vers le texte intégral en ligne.

\section{Mir@bel}

Le projet Mutualisation d'informations sur les revues et leurs accès dans les bases en ligne (Mir@bel) prolonge assez logiquement Sign@l. Initiée en 2009 par trois établissements pilotes, Sciences Po Lyon et deux de ses partenaires historiques dans Sign@l, Sciences Po Grenoble et la Bibliothèque Diderot de Lyon, la base de connaissances Mir@bel recense les informations sur les modalités d'accès en ligne au contenu des revues, notamment 
des revues francophones en SHS. Mir@bel décrit pour une revue donnée ses différents types d'accès en ligne : texte intégral en libre accès ou sur abonnement ; accès à des sommaires, à des résumés... Depuis son lancement, Mir@bel a rencontré une adhésion forte. En 2012, une nouvelle interface de Mir@bel a vu le jour. Début 2014, vingt-quatre partenaires - dont huit sont également dans Sign@l - mutualisent la veille dans Mir@bel : ils suivent 483 revues ; le moissonnage des portails couvre 1085 revues.

\section{ENCADRÉ L'INTERFACE WEB PUBLIQUE DE SIGN@L ${ }^{1}$}

L'interface Web publique de Sign@l permet de consulter l'intégralité des sommaires d'une revue ou de lancer une recherche plein texte sur le titre, le résumé ou l'auteur. Quatre modes de recherche

1. <http://doc.sciencespo-lyon.fr/Signal/>. sont proposés pour les articles : titre de la revue ; titre et/ou auteur de l'article ; date; mots-clés. Ces derniers proviennent d'un thésaurus hiérarchisé qui compte près de 2500 entrées, structurées autour de quatre catégories de descripteurs (personnes ; organismes et organisations ; lieux ; matière).

\section{ANIMER LE RÉSEAU : 2011-2014}

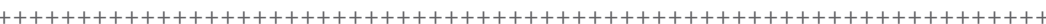

Mobilisé par Mir@bel mais également par une restructuration des espaces et des services, Sciences Po Lyon a consacré un temps réduit à Sign@l depuis 2011. Un seul partenaire a rejoint le réseau. Le partenariat avec le troisième portail de référence en SHS, Revues.org, pourrait se concrétiser en 2014.

Afin de redynamiser le réseau, Sciences Po Lyon va organiser à l'automne 2014 une réunion de tous les partenaires Sign@l. Cette rencontre sera l'occasion de définir un plan d'action pour les années suivantes. Au cours de l'année 2014, il importera également de lancer une enquête sur l'usage de la base au sein des différents établissements partenaires. 


\section{QUeLQUes PISTES À CREUSER}

Parmi les évolutions possibles de la base, deux problématiques vont surgir. Tout d'abord, se pose la question du périmètre de Sign@l : développement du moissonnage, nouveaux partenaires, ouverture aux contenus étrangers. Sur ce dernier point, on peut légitimement se demander si la richesse inexploitée de certaines revues académiques, anglophones notamment, justifie à l'heure même où le basculement vers le tout électronique se répand, que nous envisagions de saisir ou de récupérer des sommaires en langue étrangère, en anglais notamment. Faut-il tenter d'indexer certains articles issus de ces revues étrangères ? Concernant l'indexation, ensuite, on peut s'interroger sur le mode de fonctionnement actuel : faut-il maintenir le thésaurus existant, conçu, enrichi et utilisé à Lyon uniquement ? À cela s'ajoute quelques améliorations en attente devant faciliter encore et toujours l'accès au contenu des revues.

Héritier d'une activité documentaire traditionnelle, le dépouillement de périodiques, Sign@l fait partie des rares produits documentaires, né d'une initiative locale, reposant sur un modèle collaboratif, qui réussit à durer, en s'adaptant aux évolutions techniques, en intégrant les attentes nouvelles des publics tout en garantissant un libre usage des données produites dans une conjoncture qui fait la part belle aux initiatives payantes. 


\section{4}

CONCEVOIR UNE PLATE-FORME D'AUTOFORMATION

À LA RECHERCHE DOCUMENTAIRE : ARBRADOC

\section{UNE COLLABORATION ÉTROITE ENTRE ENSEIGNANTS- CHERCHEURS ET BIBLIOTHÉCAIRES-FORMATEURS}

La formation des usagers à Paris 8 s'adosse à une collaboration de longue date entre le département de documentation et la bibliothèque universitaire de Paris 8 ; il existe une unité de valeur relative à la méthodologie documentaire depuis 1985, associant de près la bibliothèque universitaire, faisant de l'université de Paris 8 l'un des premiers établissements à avoir intégré la maîtrise de l'information aux programmes disciplinaires, à la suite de la loi Savary.

Par ailleurs, une attention particulière a été portée à l'évaluation des enseignements de méthodologie par le laboratoire d'ethnométhodologie sous la direction d'Alain Coulon², dans ce qu'il nomme le processus d'affiliation des étudiants au monde académique.

\section{LE BLOG « ETUDOC » COMME PRÉFIGURATION DE LA PLATE-FORME À LA RECHERCHE DOCUMENTAIRE : LES USAGES DES ÉTUDIANTS}

Toutes deux nouvelles venues en 2006 à l'université de Paris 8, Alexandra Saemmer, maîtresse de conférences en documentation et hypermédias, notamment chargée de la coordination de l'enseignement en "Méthodologie de la recherche documentaire et du traitement de l'information », et moi-même, conservatrice en charge du service de la formation des usagers de la bibliothèque universitaire, avons pu conjointement nous

1. Responsable de la formation des usagers lors de la rédaction de cette contribution (décembre 2013).

2. Alain Coulon (dir.), L'évaluation des enseignements de méthodologie documentaire à l'Université de Paris VIII, Saint-Mandé, Association internationale de recherche ethnométhodologique, 1993. 
interroger sur la pédagogie de cet enseignement, dans le nouvel environnement numérique apparu au cours de ces dernières décennies.

Prenant en compte le déploiement radical du document vers le Web, il nous a semblé nécessaire de prendre pour point de départ les usages " réels » des étudiants, plutôt que les prescriptions idéalement circonscrites dans le monde académique, ce dont nous avons fait part dans une communication lors d'une journée d'étude à Médiaquitaine en avril 2009. Dans cette tentative de "rénovation " pédagogique laissant la part belle à l'« affordance »* et à la sensibilisation au numérique, Alexandra Saemmer a pris le parti d'offrir aux enseignants-documentalistes en charge de cet European Credit Transfer System (ECTS), un espace de plate-forme où déposer leurs cours, sous la forme d'un blog ouvert à tous, Etudoc ${ }^{3}$. À la consultation des statistiques, il s'est avéré que la fréquentation en était importante, et que son offre de formation pourvoyait à un véritable besoin méthodologique.

À l'analyse des contenus déposés sur le blog, il était aussi notable que chaque enseignant reprenait peu ou prou les mêmes aspects méthodologiques, et que leur mise à plat apporterait une véritable aide en ligne aux étudiants.

\section{PROBLÉMATIQUE DU SERVICE DE LA FORMATION DES USAGERS DE LA BIBLIOTHĖQUE UNIVERSITAIRE}

Parallèlement, le service de la formation des usagers recevait l'injonction hiérarchique de former toujours plus d'étudiants, à moyens humains et matériels constants. Il est vite apparu que disposant d'une salle de formation dédiée et équipée, et d'une équipe volontaire d'une dizaine de bibliothécaires-formateurs, nous ne pouvions offrir des formations de qualité au-delà de 2000 étudiants formés par an. Le recours à des tuteurs-étudiants pour une formation à plus grande échelle ne pouvait se faire sans une perte effective de qualité. 


\section{LIER L'ACCÈS AUX DOCUMENTS À LEUR UTILISATION ACADÉMIQUE RÉELLE}

Bien trop souvent la formation des usagers dispensée par les bibliothèques universitaires est focalisée sur l'accès aux documents via les différentes bases de données, les procédures de requête, les langages documentaires, etc. Une certaine complaisance incline les professionnels des bibliothèques à s'enfermer dans un langage technique, coupé de la véritable raison d'être du document, à savoir son appropriation par les étudiants. L'accès aux documents n'apparaît alors pas lié au besoin documentaire qui motive sa recherche, et la masse d'informations devient arbitraire et indifférenciée aux yeux des étudiants.

Du partenariat de longue date établi entre bibliothécaires et enseignantsdocumentalistes, il ressort une attention portée à l'imbrication de la formation à la culture informationnelle dans la méthodologie du travail universitaire.

\section{Recherche de financement de projet, la saisie d'une opportunité}

Le plan « Réussir en licence » initié par la ministre Valérie Pécresse en 2008 a doté les universités d'enveloppes budgétaires substantielles pour lutter contre le taux d'échec important lors des premières années universitaires. Une action de " remédiation » portant sur la documentation étant comprise dans les textes, comme favorisant la réussite des étudiants, cela a permis à la bibliothèque universitaire de présenter un projet, voté au Conseil des études et de la vie universitaire (CEVU) en 2008, afin de réaliser une plateforme d'autoformation à la recherche documentaire en libre accès.

Le budget qui a été alloué pour ce projet a été de 30000 euros au total sur deux années budgétaires (2008 et 2009). La répartition des postes budgétaires sur deux années consécutives est à peu près la suivante : la prestation de développement a été rétribuée de 14000 euros TTC ; le graphisme de l'interface et son intégration sont revenus à 4000 euros TTC; la rédaction des préconisations techniques a été rétribuée pour 2000 euros bruts, ainsi que la trentaine de cours rétribués pour 10000 euros bruts à la dizaine de rédacteurs participants. 


\section{DE LA CONCEPTION À LA RÉALISATION DE LA PLATE-FORME}

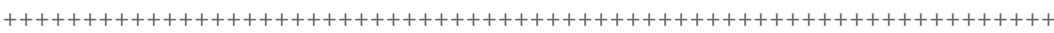

Disposant de cette manne financière obtenue quasiment sur une note d'intention plus qu'un projet véritablement écrit, il nous a fallu commencer par la rédaction du cahier des charges fonctionnelles.

Une étudiante-stagiaire suivant la formation de l'Institut national des techniques de la documentation (INTD) a accompli un très beau travail, consistant en un état de l'art des référentiels de compétences informationnelles, ainsi que des différents tutoriels de recherche documentaire. Sur la base de cette solide évaluation des besoins et de l'existant, elle a pu rédiger un cahier des charges fonctionnel rigoureux, précieux appui pour mener le projet au terme de sa réalisation.

Enfin, ses compétences en ingénierie documentaire nous ont permis de nous orienter vers la chaîne éditoriale libre Scenari ${ }^{4}$, afin de produire des contenus en ligne normés et structurés, bien qu'il y ait de nombreux rédacteurs. Ce cahier des charges est lui-même accompagné d'une réflexion plus large menée dans un mémoire ${ }^{5}$ sur la formation à l'information à l'ère du numérique.

\section{LA COORDINATION ET LA FORMATION DES RÉDACTEURS DE CONTENUS}

Il était convenu à l'orée du projet que cette plate-forme devait refléter la collaboration étroite entre enseignement de méthodologie (enseignantschercheurs) et formation à la recherche documentaire (bibliothécairesformateurs), afin de conjuguer l'expérience et la pédagogie des uns et des autres.

L'équipe de rédacteurs était ainsi constituée de bibliothécaires (tous expérimentés dans la formation des étudiants) et d'enseignants-chercheurs chargés de cours de méthodologie. Par ailleurs, l'université de Paris 8 étant dotée d'un axe fort de recherche en hypermédias, des

4. < http://scenari-platform.org >.

5. Aurélie Puybonnieux, Se former à l'ère du numérique : nouveaux enjeux et nouveaux acteurs de la formation à l'information dans les bibliothèques universitaires. Le cas de l'Université de Paris 8, Mémoire de master : chef de projet en ingénierie documentaire, INTD, 2010. [En ligne] : < http:// memsic.ccsd.cnrs.fr/docs/00/57/50/61/PDF/PUYBONNIEUX.pdf >. 
enseignants-chercheurs de ce domaine sont venus se greffer au projet afin de proposer des cours de culture numérique.

Une session de formation de deux jours à la prise en main de la chaîne éditoriale Opale Sup Scenari a été organisée gracieusement sur site par Olivier Poinsart, animateur de la communauté Scenari. Il nous a accompagnés tout au long du projet, notamment pour guider les deux informaticiens de la bibliothèque dans l'installation et le bon fonctionnement du serveur Scenari.

\section{L'INTERFACE D'ACCÈS AUX COURS, LE CHOIX DE LA CARTE HEURISTIQUE COMME OUTIL DE VISUALISATION PÉDAGOGIQUE}

De façon assez précoce dans le projet, il nous a semblé important que le design de la plate-forme permette une compréhension organique des liens des cours entre eux et de leur complémentarité, dans une forme de logique qui puisse être embrassée d'un seul coup d'œil. La plate-forme de cours étant conçue comme un outil d'apprentissage informel et autodidacte, la représentation arborescente et la disposition des cours entre eux devaient mener l'étudiant à une meilleure perception de son besoin documentaire.

Il est à noter qu'entre-temps on a pu assister à l'éclosion d'autres usages des schémas cognitifs dans la compréhension des compétences informationnelles, ce qui paraît tout à fait convaincant comme mode d'orientation implicite de l'usager, notamment avec le référentiel de compétences proposé par l'Association des directeurs de bibliothèques universitaires (ADBU).

L'arborescence de l'interface d'Arbradoc est composée de quatre branches sur lesquelles il faut cliquer pour faire apparaître les items de chacun des cours, eux-mêmes organisés par rubriques, qui, elles, apparaissent en caractères rouges, non cliquables.

Alors que le « Parcours fléché » déploie les différentes étapes d’une recherche documentaire, de l'analyse du sujet à l'exploitation des documents, le « Parcours libre » entre plus en profondeur dans la recherche par type de documents, et ordonne ces différents types selon un besoin documentaire aligné sur les différents travaux universitaires. Cette logique en cascade, de la recherche documentaire tendant vers l'accès aux documents, 
est poursuivie avec la branche " Outils de recherche ", qui propose des tutoriels spécifiques aux bases de données présentes tout au long des cours. Enfin, un parcours d'autoévaluation est aussi accessible à ce niveau.

Par ailleurs, une autre branche est venue s'adjoindre, celle de la « Culture numérique ", proposant des outils et des concepts à travers l'analyse d'œuvres numériques.

\section{CAPTURE D'ÉCRAN LES PRINCIPALES BRANCHES D'ARBRADOC}

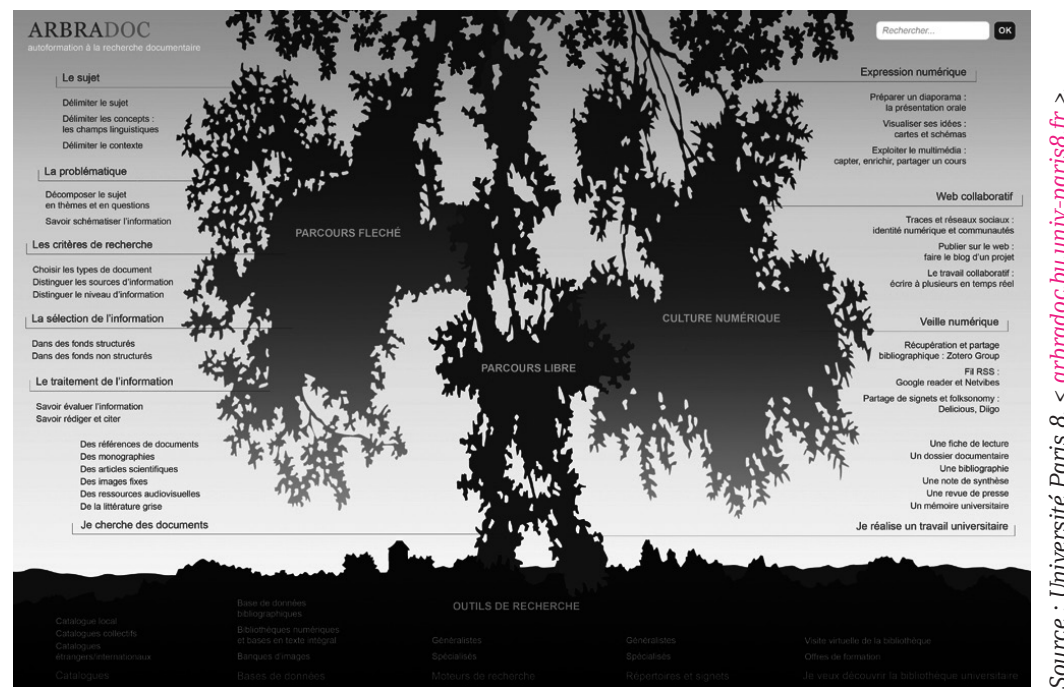

RÉSOLUTION TECHNIQUE DE L'ACCÈS PAR UNE INTERFACE UNIQUE

La chaîne éditoriale Opale Sup Scenari présentait jusqu'alors une solution d'éditorialisation des unités de cours sur différents supports, sans proposer de solution de type " plate-forme » permettant d'organiser de façon hiérarchique la totalité des cours pris ensemble. Au cours de notre progression, nous avons recherché quelles étaient les différentes sortes de plates-formes, de type Moodle*, permettant à la fois la gestion et le stockage des cours, mais aussi leur mise en accès sur le Web, via une interface de type carte heuristique. Un doctorant en informatique a produit des 
préconisations pour une solution technique, nous permettant de rédiger un appel d'offres dans le cadre des marchés publics.

La solution retenue a été celle de synchroniser les contenus déposés sur le serveur Scenari de la bibliothèque universitaire vers un CMS Drupal, et de procéder à un développement à cette fin, le CMS Drupal étant en open source*. Nous étions orientés dans ce choix du fait qu'un projet de refonte du portail de la bibliothèque avec le CMS Drupal assurait quelques compétences en interne, au service informatique de la Bibliothèque, pour la maintenance de la plate-forme.

\section{COORDINATION DES RÉDACTEURS, DE L'INFORMATICIEN ET DES FORMATEURS}

Une fois le prestataire de développement Drupal retenu suite à un appel d'offres dans le cadre des marchés publics, nous avons lancé conjointement la phase de rédaction des contenus sur le serveur Scenari et la phase de développement par le prestataire d'une plate-forme permettant de synchroniser ces mêmes contenus en ligne. Commande a été passée au peintre-dessinateur Richard Müller d'un dessin sous forme de calque, représentant un arbre, dont les contours ont été vectorisés et retravaillés par un graphiste ; une fois ce dessin rendu numérique, il a été intégré au CMS Drupal comme interface d'accès, dont les liens pointaient vers les cours importés du serveur Scenari.

L'équipe des bibliothécaires-formateurs a surtout été mise à contribution pour les tutoriels des bases de données dont elle avait l'habitude d'assurer la présentation. Bien que rétribués, certes modestement, il n'a pas toujours été évident de motiver les enseignants-chercheurs, déjà accaparés par leurs propres publications, à produire des contenus. Pour ma part, j'ai eu la satisfaction de pouvoir inscrire ma démarche pédagogique dans un cours, "Rechercher des références bibliographiques ", partant de la recherche sur le Web visible jusqu'à la valeur ajoutée documentaire des bases de données, ce à quoi il est vraiment important de sensibiliser les étudiants. Lorsque nous avons commencé à pouvoir " charger » les cours dans la plate-forme, nous avons dû alors faire des allers-retours importants avec le prestataire de développement informatique, afin de procéder à des corrections et à des réglages du bon fonctionnement de la plate-forme ; les 
exercices interactifs de Scenari ont notamment eu beaucoup de difficulté à être synchronisés, défaut persistant.

Mise en ligne le 23 janvier 2012, la plate-forme Arbradoc a reçu depuis lors environ 18000 visiteurs, pour une durée moyenne très courte... C'est à ce moment que j'ai passé le relais à d'autres expérimentations pédagogiques à inventer, toujours en collaboration entre bibliothécaires et enseignants-chercheurs : la plate-forme offrant un contenu très dense, il me semble qu'une intégration des contenus dans les cours est nécessaire, alliant pédagogie en présentiel et autodidaxie en distanciel, ce qui laisse de beaux jours au partenariat entre bibliothécaires et enseignants. 


\section{5}

INTÉGRATION DES FONCTIONNALITÉS D'OPENCAT MUNICIPALE DE FRESNES

\section{DATA.BNF.FR}

Le site data.bnf.fr ${ }^{1}$, mis en ligne en juillet 2011, fédère différentes ressources de la $\mathrm{BnF}$ issues de plusieurs bases de données et intègre des liens vers des ressources externes, partenaires institutionnels et sites extérieurs. Il se présente sous la forme de pages Web interrogeables par les internautes, entre autres au moyen des moteurs de recherche du Web. Data.bnf.fr participe ainsi de ce qu'il est coutume d'appeler le « Web de données », rendant accessibles des données généralement cachées (Web profond). En juin 2013, le volume du site data.bnf.fr représentait $40 \%$ des catalogues de la BnF.

Dans le courant de l'année 2014, plusieurs aménagements fonctionnels sont prévus et en 2015, $80 \%$ des notices bibliographiques du catalogue général seront accessibles sur le site, soit la totalité des notices bibliographiques liées à une notice d'autorité validée.

Quels services peut-on construire à partir de la récupération de ces données librement et gratuitement réutilisables?

\section{LA BM DE FRESNES : L'EXPÉRIMENTATION OPENCAT}

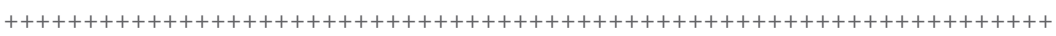

Dès sa première informatisation en 1992, la bibliothèque de Fresnes a construit son catalogue à partir des notices bibliographiques et d'autorité de la $\mathrm{BnF}$ au format INTERMARC, évitant les pertes d'information provoquées

1. Depuis le $1^{\mathrm{er}}$ janvier 2014, la BnF a placé l'ensemble de ses métadonnées descriptives (bibliographiques et d'autorité) sous la licence ouverte de l'État : < etalab.gouv.fr/licence-ouverteopen-licence $>$. 
par les conversions de format. Le fichier d'autorité a été intégralement récupéré et mis régulièrement à jour. Les notices bibliographiques produites localement ou récupérées ont été par la suite automatiquement écrasées et remplacées par les notices de la $\mathrm{BnF}$ au moyen du "vendangeur » de la société Opsys en reproduisant les liens entre les différents types de notices indexées. La base disposait donc d'un mode de fonctionnement et des identifiants $\mathrm{ARK}^{* 2}$ nécessaires à l'établissement des liens avec data.bnf.fr. En 2012, cette structuration a permis à la ville de Fresnes de répondre conjointement avec la $\mathrm{BnF}$ et la société Logilab à l'appel à projets du ministère de la Culture et de la Communication « Services numériques culturels innovants », pour mener l'expérimentation OpenCat.

LE PROTOTYPE ${ }^{3}$ ISSU DE CETTE EXPÉRIMENTATION COMPREND :

- les regroupements par œuvres réalisés pour le site data.bnf.fr ;

- une partie des données locales de la bibliothèque municipale de Fresnes (littérature française, musique) soit environ 4000 œuvres communes avec data.bnf.fr ;

- des compléments bibliographiques absents des deux premières bases, comme les notices critiques du Centre national de littérature pour la jeunesse ;

- l'agrégation de données non-bibliographiques : vignettes de couverture, portraits d'auteurs, informations biographiques ;

- des liens directs vers des ressources en ligne multimédia provenant de Gallica, d'expositions virtuelles de la BnF, de conférences scientifiques de Canal-U, etc.

Les acquis de cette expérimentation ont été conservés selon une autre présentation dans la nouvelle version du catalogue en ligne de la bibliothèque ${ }^{4}$, actuellement en phase d'expérimentation. Ce développement, ainsi que sa conception, sont réalisés en interne, et c'est une première

2. Plus d'informations sur les ARK à la BnF : < http://www.bnf.fr/fr/professionnels/issn_isbn_ autres_numeros/a.ark.html >.

3. < http://demo.cubicweb.org/opencatfresnes/ >.

4. < http://bm.fresnes94.fr >. 
phase de cette expérimentation, l'agrégation des données issues de data. bnf.fr, que nous vous présentons ici.

\section{DES PRÉREQUIS TECHNIQUES}

Les bibliothèques doivent s'assurer d'avoir accès à une interface de programmation implémentée dans leur logiciel de gestion, seul moyen d'exploiter leurs données en vue du développement évolutif d'un catalogue en ligne. Cet accès est souvent difficile, voire absent. Le prestataire considérant qu'il s'agit d'une option, ou le considérant comme secondaire, il n'offre alors que des fonctionnalités de base qui sont difficilement adaptables aux standards d'aujourd'hui. De plus, les technologies sous-jacentes aux bases de données, parfois vieillissantes, certes complètes et adaptées à des structures complexes, sont lourdes à exploiter alors que l'on se dirige toujours plus vers la simplification des formats. Tout ceci limite et/ ou alourdit les requêtes et implique généralement pour les concepteurs une réécriture complète de leur solution. Enfin, beaucoup de systèmes proposent un portail documentaire intégré, donc fermé, à l'évolution très difficile voire impossible.

Un système intégré de gestion de bibliothèque (SIGB) ne se limite pas à l'accès au catalogue, c'est un système de gestion complet qui doit être capable de gérer des usagers, des flux de documents, des processus métiers complexes capables de s'adapter aux protocoles mis en place par l'établissement (circuit du document en adéquation avec la politique d'acquisition documentaire notamment), mais doit aussi être capable de fournir une synthèse de ces processus et de leurs flux pour en faire une exploitation statistique déportée dans une présentation simplifiée.

Dans le meilleur des cas, un SIGB va autoriser l'accès aux données du catalogue par l'intermédiaire d'une Application Programming Interface (API ou Interface de programmation). Celle-ci va généralement exposer des méthodes ou fonctions via un service Web (mode de transport HTTP). Il suffit là de comprendre que les données échangées entre le client et le serveur sont transportées par le même protocole que celui utilisé pour le Web, HTTP, originellement utilisé pour transférer du code de balises HTML. Nous partons du principe que les données du catalogue de la bibliothèque sont entièrement accessibles par une application tierce, en l'occurrence 
un portail documentaire sous la forme d'une application Web, et que cet accès est sans restriction, tant en lecture qu'en écriture.

\section{UNE REQUÊTE SUR DATA.BNF.FR}

Data.bnf.fr agrège les données des différents catalogues de la BnF, exposées de plusieurs manières. Il y a la partie émergée, visible, où autorités et œuvres sont alignées et présentées sur une simple page Web. Et pour un alignement et une agrégation de ces données sur le catalogue en ligne de la bibliothèque, un accès exploitable par une machine.

Il est aujourd'hui possible de télécharger tout ou partie de data.bnf.fr via des dumps, copies de la base de données, et de s'y servir localement, solution qui implique un téléchargement initial volumineux et des mises à jour régulières ${ }^{5}$. La bibliothèque de Fresnes a de son côté testé les possibilités de récupération dynamique d'informations depuis le site. La première version de data.bnf.fr n'était cependant pas prévue pour répondre dynamiquement à des requêtes, fonctionnalité qui sera remplie en 2014 par la mise en place d'un point d'accès SPARQL* ("SPARQL-endpoint ", permettant des requêtes sous forme de "triplets " $\mathrm{RDF}^{*}$ dans les standards du Web sémantique $)^{6}$. Les modalités de récupération dynamique d'informations sont donc amenées à évoluer, mais la philosophie de récupération décrite ci-dessous reste la même.

5. Plus d'informations : < http://data.bnf.fr/semanticweb\#Ancre2 >.

6. Pour aller plus loin, un ensemble de ressources est disponible : < http://www.w3.org/2004/01/ sws-pressrelease.html.fr >. Pour tester des requêtes en SPARQL sur dbpedia : < http://dbpedia. org/sparql >. 


\section{FIGURE LE PROCESSUS GLOBAL DE REQUÊTE}

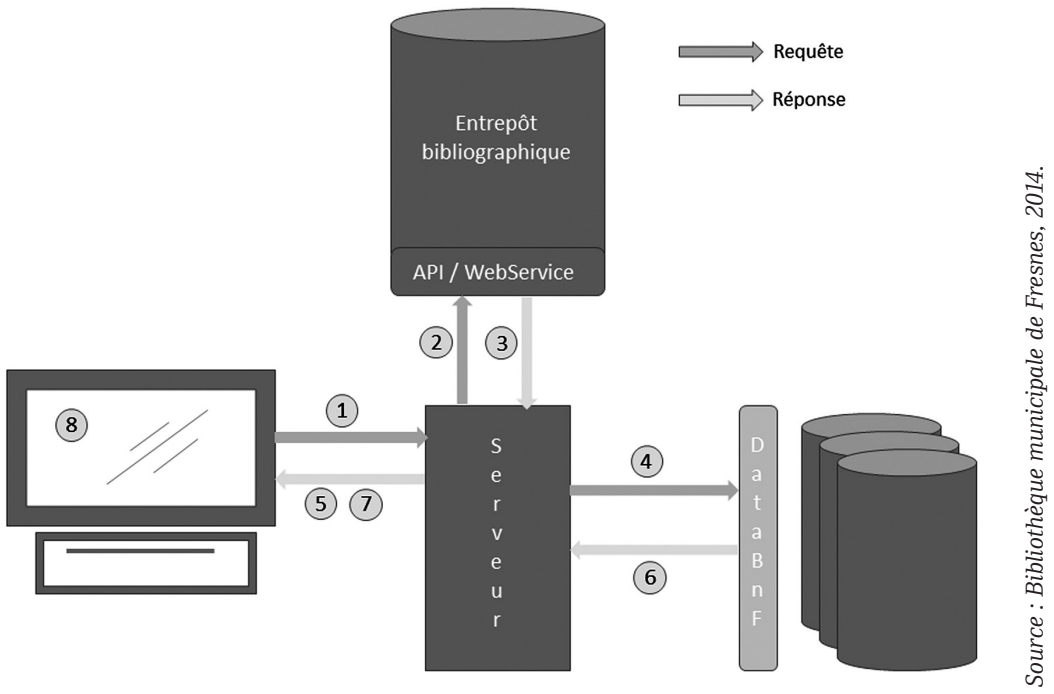

Légende des étapes

1. l'internaute envoie une requête au serveur par mot-clé ;

2. le serveur se charge d'interroger le service WEB attaché à l'entrepôt bibliographique ;

3. le service bibliographique répond au serveur ;

4. dès que le numéro de la notice $\mathrm{BnF}$ est connu, une requête est envoyée à data.bnf.fr pour gérer la négociation de contenu ;
5. simultanément (les requêtes sont asynchrones) le serveur envoie les premiers éléments de la réponse au client ;

6. la réponse de data.bnf.fr est reçue ;

7. le serveur répond au client avec les derniers éléments glanés depuis data.bnf.fr ;

8. la page constituée s'affiche sur l'écran de l'internaute. 
PRENONS POUR EXEMPLE JULES VERNE. L'URI* DE CET AUTEUR SUR DATA.BNF.FR EST : 〈HTTP://DATA.BNF.FR/11928016/JULES_VERNE/〉.

- Au moment de la requête, nous ne disposons que du numéro de la notice. Il faut aujourd'hui « discuter » ou « négocier » avec le serveur de la BnF car il est impossible d'anticiper la syntaxe exacte du nom de l'auteur, le prénom devant parfois être intégré dans l'URI finale. Cette négociation est prévue dans le protocole de requête "XmlHttpRequest ». Il s'agit du preflight, requête qui va établir la liaison "inter-domaine ». Elle reçoit en réponse du serveur (le fournisseur) l'URI de la demande complète. Cette URI renvoyant vers une page HTML, il faut concaténer une extension au nom de l'autorité, afin d'obtenir le fichier de description de l'objet de la requête, et de le manipuler en vue d'en exposer les données.

- Après concaténation de l'extension à l'URI et une nouvelle requête, il devient possible de récupérer les données sous la forme d'un fichier. Ainsi, dans notre exemple, l'URI de la demande finale devient : < http://data.bnf.fr/11928016/jules_verne.rdf > ou < http://data.bnf.fr/11928016/jules_verne.json > qui sont les deux formats exploitables pour le moment.

- Le format JSON est moins complet, mais les informations sont suffisantes pour l'utilisation souhaitée dans ce cas de figure (données d'autorité), et c'est donc ce fichier que nous allons récupérer et exploiter par souci de rapidité de l'affichage, la réponse au fichier RDF étant plus longue.

En réponse, data.bnf.fr renvoie le fichier à analyser pour exposer son contenu sur le catalogue en ligne. Nous avons alors accès aux données relatives à l'auteur telles que décrites sur data.bnf.fr (type de ressource, provenance, dates de naissance et de mort, formes rejetées, lien vers la vignette associée provenant de Gallica ou de Wikipédia, notes relatives à l'auteur, tableau de ses œuvres avec leur type et leur date ainsi qu'un lien constitué de l'URI de chaque œuvre pointant sur data.bnf.fr). Les œuvres sont alors exposées sur le catalogue sous forme de frise chronologique, comme c'est le cas dans l'expérimentation OpenCat. En plus d'être ludique, d'attirer le regard et donc d'attiser l'intérêt, cette présentation particulière a l'avantage de replacer l'œuvre dans son contexte historique. 


\section{CAPTURE D'ÉCRAN}

CATALOGUE EN LIGNE DE LA BIBLIOTHĖQUE DE FRESNES

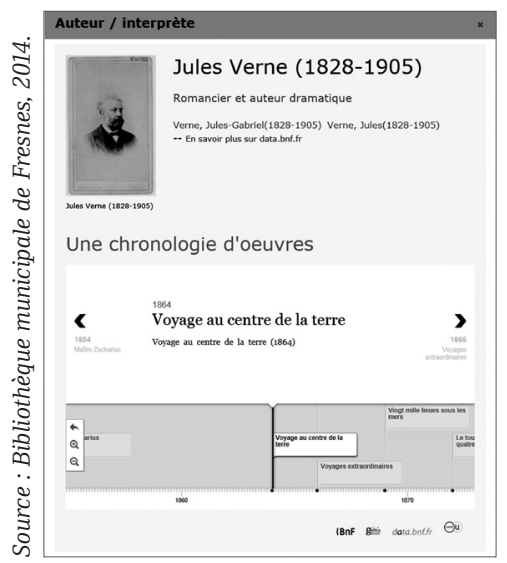

Les données ainsi récupérées sont utilisées pour agréger le contenu dans la fiche correspondante sur le portail documentaire. Le portrait de l'auteur, les notes afférentes et ses formes rejetées sont présentés à l'internaute qui aura cliqué sur l'autorité depuis la fiche d'une notice de «manifestation $»^{7}$.

\section{Le modèle Functional Requirements for Bibliographic Records (FRBR)*.}

Les requêtes de base sur le catalogue du portail documentaire renvoient sur les éditions (" manifestations » selon le modèle FRBR) dont au moins un exemplaire est présent dans le fonds de la bibliothèque. La frise chronologique permet d'accéder aux œuvres. Pour le moment, les liens attachés renvoient sur l'URI de description sur data.bnf.fr. À terme, il est prévu de relier ces œuvres à leurs " manifestations ». Nous nous appuierons pour cela sur le modèle FRBR.

L'idée générale derrière les FRBR est qu'un texte original, sa traduction, une adaptation de l'œuvre renvoient tous vers une même entité abstraite. Pour cela, quatre " aspects " rattachés à cette entité sont définis, ici avec notre Voyage au centre de la terre pour servir d'exemple.

7. Selon le modèle FRBR, voir infra. 
- Euvre : Voyage au centre de la terre de Jules Verne

- Il s'agit de l'entité la plus abstraite, l'œuvre en tant que création intellectuelle. Une œuvre musicale par exemple n'a pas d'identité tant qu'elle n'existe pas sous la forme d'une « expression » ou d'une «manifestation »-partition, concert, disque ;

- Expression : Journey to the center of the earth - adaptation cinématographique de l'œuvre, de Henry Levin, 1959 ;

- Manifestation : Voyage au centre de la terre, Paris : l'École des loisirs, 1980 - Donc une édition particulière. Le manuscrit original est aussi l'une des manifestations de l'œuvre ;

- Item : un exemplaire de la manifestation se trouvant à la bibliothèque de Fresnes.

- Cela constitue donc un ensemble hiérarchisé qui va du plus abstrait (l'œuvre) au plus précis (l'item).

Pour pouvoir exploiter les liens dans cette structure particulière, il est nécessaire que le SIGB prenne en charge ce modèle. En effet, depuis l'œuvre décrite par data.bnf.fr et récupérée pour constituer la frise chronologique, il faut, pour "redescendre » vers les « manifestations », puis par extension aux « items ", que l'entrepôt de données soit à même de créer ces liens et de les certifier. L'ouverture des métadonnées de la BnF depuis le $1^{\text {er }}$ janvier 2014 devrait grandement aider à les établir.

Cette première phase de l'expérimentation menée à la BM de Fresnes tend vers une homogénéité des données agrégées, en exploitant du contenu issu de sources " certifiées ». En effet, les sources sont diverses sur le Web de données, mais il est peut-être encore trop tôt pour les exploiter pleinement. Wikipédia par exemple, permet une interrogation « sémantique » de leur base de données, mais celle-ci ne peut se faire pour le moment qu'à partir du nom des autorités, et ne gère pas la recherche approximative. Cela induit des erreurs liées aux homonymies. À terme, il apparaît donc nécessaire que les fournisseurs de contenus utilisent les ontologies rattachées au Web sémantique prévues pour créer des liens entre les ressources issues de sources différentes ("owl:sameAs"). Ainsi, chaque entité décrite sur Wikipédia pourrait avoir son équivalent lié à une autorité décrite par 
la BnF. Ces liens ne sont pas forcément fiables et pour le moment, il faut considérer qu'il existe une autorégulation, telle que celle qui a lieu sur Wikipédia. D’autre part, l'utilisation de standards internationaux, tel que l'International Standard Name Identifier (ISNI) ${ }^{8}$, peut garantir la fiabilité des liens entre autorités. La BnF, membre de l'agence qui régule ce standard depuis 2010, est la première bibliothèque à avoir chargé des ISNI (près d'un million) dans son catalogue. Wikipédia a commencé à déployer ces mêmes identifiants dans sa base de données par un alignement avec Virtual International Authority File (VIAF, Fichier d'autorité international virtuel), mis en œuvre et hébergé par OCLC. 134380 ISNI sont ainsi déjà intégrés à Wikipédia EN, mais seule une fraction est actuellement alignée sur Wikipédia FR, grâce aux liens inter-langues.

Une volonté politique d'ouvrir les données publiques existe désormais, comme le montre la mission interministérielle Etalab, service du Premier Ministre qui a donné naissance fin 2011 au portail de données ouvertes data.gouv.fr. L'ouverture des données implique une mise à disposition de celles-ci au plus grand nombre, mais seul un accès standardisé et affranchi des formats propriétaires peut en garantir l'exploitation par des systèmes informatiques tiers. Pour cela, il est essentiel que les entrepôts de données soient eux-mêmes structurés dès l'origine dans des standards ouverts. En ce sens, le travail mené par la BnF est remarquable.

8. Identifiant unique et pérenne pour les créateurs. Plus d’informations : < http://www.bnf.fr/fr/ professionnels/issn_isbn_autres_numeros/a.isni.html >. 


\section{PARTIE Th}

\section{LES PRODUITS}

DOCUMENTAIRES DE

SYNTHĖSE : CURATION

ET PRODUCTION

1. LES DOSSIERS DOCUMENTAIRES NUMÉRIQUES DE LA MÉDIATHÈQUE INTERCOMMUNALE OUEST PROVENCE par Jérôme Pouchol

2. LES AGRÉGATEURS DE CONTENUS ET LA CURATION

À LA MÉDIATHĖQUE DE BAGNOLET

par Dominique Macé

3. NUMÉRISATION DE MASSE, MÉTADONNÉES ET BIBLIOTHĖQUE NUMÉRIQUE : L'EXEMPLE DE NUMELYO À LA BIBLIOTHĖQUE MUNICIPALE DE LYON

par Magali Haettiger

$+++++++++++++++++++++++++++++++++++++++++++++++++++++$

4. BALISES, LE WEBMAGAZINE DE LA BIBLIOTHĖQUE PUBLIQUE D'INFORMATION

par Nathalie Nosny 


\section{1}

\section{LES DOSSIERS DOCUMENTAIRES NUMÉRIQUES DE LA MÉDIATHĖQUE INTERCOMMUNALE OUEST PROVENCE} Jérôme

\section{DE NOUVELLES PRATIQUES DE LECTURE}

Bien que la surcharge informationnelle ne soit pas née avec le Web, ce dernier a considérablement amplifié notre sentiment d'être noyé dans un flux exponentiel de données que nos cerveaux ne peuvent désormais plus traiter. De cette surabondance, l'internaute informavore construit des anthologies, déploie des stratégies plus ou moins élaborées de veille informationnelle, développe des réflexes de lecture écrémage, de lecture fragmentée, de lecture de scrutation ; en somme, une pratique active et impérieuse de pré-lecture.

Dans ces nouveaux " arts de faire lecture ", le produit de synthèse* trouve naturellement sa place, en ce qu'il répond au besoin d'appréhender l'essentiel d'un sujet, de structurer et de mémoriser des connaissances dans une économie de temps et de moyens heuristiques. Les secteurs de la presse et de l'édition l'ont bien compris, qui rivalisent de productions synthétiques diversiformes. Dans le domaine de la pédagogie et de la formation, l'aptitude à synthétiser est un savoir-faire particulièrement mobilisé et évalué, désormais placé au cœur des méthodes et objectifs pédagogiques.

Dans ces différents cadres, les spécialistes du traitement de l'information que sont les documentalistes et les bibliothécaires sont parmi les professionnels les mieux à même de fournir une offre de qualité, en associant la maîtrise d'un savoir-faire, la fourniture de contenus et des moyens technologiques idoines.

Au sein de la Médiathèque intercommunale Ouest Provence (MIOP), nous verrons que les dossiers documentaires - dans leur fonction essentiellement « outil »- peuvent servir plusieurs objectifs ou cibles, tout en visant à intéresser le plus grand nombre d'utilisateurs possibles ; nous 
considérerons également la valeur ajoutée qu'ils offrent à leurs auteurs, à ceux qui les élaborent et en assurent la diffusion numérique et ce, toujours en relation étroite avec les collections physiques dont ils ont la charge.

\section{QUELLES SONT LES CARACTÉRISTIQUES DU DOSSIER DOCUMENTAIRE ?}

Le dossier documentaire a principalement valeur de synthèse, au sens où il organise un savoir en rassemblant, en un tout homogène, divers éléments sélectionnés dans plusieurs sources d'information hétérogènes.

C'est aussi, sur le plan des contenus, un document à forte valeur ajoutée, qui devra s'appuyer sur une exigence de fiabilité des contenus sélectionnés ; actualité des informations fournies ; pertinence du contenu, soit conforme à la « cible » du sujet comme à son cadre de synthèse, soit complémentaire dans la diversité des documents et médias sélectionnés ; cohérence et rigueur intellectuelles.

Le corpus du dossier est formé de documents primaires et secondaires, l'association de ces deux éléments dans une forme originale lui conférant un statut de document dit " tertiaire », désigné autrement par le terme de " produit documentaire ».

Sa forme électronique permet de proposer plusieurs niveaux de profondeur et de cheminement de lecture, évitant de surcharger la " première » page d'une masse trop importante d'informations pouvant faire obstacle à une compréhension globale du sujet.

L'architecture du dossier favorise également un type de navigation par rebonds en agrégeant et en structurant les contenus par portions d'informations complémentaires, lesquelles sont autant de points d'accès à des savoirs plus étendus. L'organisation de ces éléments sert une intelligibilité de l'information dans son ensemble, en considérant que le tout est supérieur à la somme des parties qui le constituent.

L'objet dossier gagnera alors à présenter une architecture graphique commune, une approche de canevas d'édition facilitatrice tant pour son propre artisan que pour son utilisateur. 


\section{organisation en modules et forme rédactionnelle}

À la MIOP, le dossier documentaire est rubriqué en modules (ou widgets*) distincts qui portent une fonction particulière. Ces modules autonomes sont transférables à l'ensemble des dossiers, quelles que soient les thématiques retenues. Ils forment ainsi tout à la fois un corpus homogène de documents et une interface graphique et logique communes, plus aisément identifiable sur le portail documentaire du réseau.

Chaque module - en particulier ceux contenant du rédactionnel - doit répondre aux exigences de forme propres à l'environnement Web, en se conformant à des critères de :

- concision rédactionnelle : technique d'écriture en « chapeaux », phrases simples sans relatives, paragraphes courts, vocabulaire intelligible, introduction liminaire ;

- lisibilité graphique : corps et couleur de fonte, cohérence de la casse, choix des polices, interlignage, usage du gras pour les termes importants, listes à puces ou numérotées ;

- design et ergonomie informationnels : forme et taille des blocs, espace entre les modules, qualité et positionnement des images, intuitivité de navigation, gestion des hyperliens et du défilement.

À ces consignes de forme s'ajoute celle du bon respect des règles linguistiques. L'auteur du dossier se montrera également très vigilant sur la question des droits de la propriété intellectuelle et artistique en ne proposant que des contenus strictement libres de droit.

\section{Prescriptions de contenu préalables à la constitution d'un dossier}

Pour le choix du sujet, appliquer les " lois de proximité », soit le principe que le lecteur est avant tout attiré par ce qui le concerne directement. Privilégier le présent d'actualité (proximité temporelle) ainsi que des thématiques qui interpellent le lecteur « collectivement », dans ses préoccupations sociales du moment (proximité sociale). 
Délimiter le champ de traitement du sujet. Une conception trop large de la thématique choisie lui fera perdre de son utilité pour l'usager. A contrario, une approche trop spécialisée du sujet risque d'intéresser un segment trop restreint de lecteurs.

Le dossier est porteur d'une intentionnalité, il ne saurait être neutre - tant par le choix du thème que par la sélection des éléments qu'il contient - mais se veut pluraliste, en ce qu'il doit rendre compte de la diversité des approches constatées sur le sujet.

Le sujet doit être abordé sous un angle dialectique, voire critique, afin d'envisager d'emblée les controverses qu'il peut susciter. Le dossier gagne ainsi à problématiser le sujet, à ne pas se limiter à une démarche exploratoire ou à une addition de données. Il peut, à cet égard, être " pensé » comme si l'auteur devait le présenter en conférence, auprès d'un auditoire intéressé par le sujet.

\section{Détail et illustration des différents modules des dossiers de la MIop ${ }^{1}$}

\section{Consignes générales}

Usage des paragraphes :

- leur titre (statistiquement 5 fois plus lu que le texte) est court (5 mots maximum), informatif et riche en mots-clés ;

- éviter les appositions et les mises en route trop longues en début de chapeau ;

- ne consacrer de préférence qu'une seule idée principale par paragraphe ;

- ne pas dépasser 5 paragraphes, soit 5 notions essentielles ;

- limiter la rédaction à 10 lignes maximum (au mieux, 5), pour ne pas " perdre » le lecteur dès les premières lignes de lecture, ni déséquilibrer l'interface d'ensemble du dossier ;

1. < www.mediathequeouestprovence.fr/index.php?id=3145 >. 
- gestion des hyperliens : leur usage est privilégié, pour compenser le caractère liminaire du texte et favoriser une pratique interactive de sa lecture.

Usage des images :

- pour une mise en valeur du contenu... ;

- une légende est ajoutée si le contexte ne suffit pas à les rendre explicites ;

- elles sont ajustées à la taille d'affichage ;

- leurs poids, format et qualité de résolution sont, si possible, normalisés et/ou automatisés par l'outil CMS.

Autres consignes :

- placer les mots-clés et informations principales en début de phrase ;

- préférer un style direct et des phrases courtes. Seuls 15/20 mots par phrase sont mémorisables à la lecture ;

- utiliser la voie active plutôt que la voie passive ;

- ne pas dépasser 15 lignes d'écriture maximum ;

- accompagner le texte d'une image (signifiante et/ou esthétiquement valorisante).

Le titre du dossier : il doit être percutant, explicite pour le lecteur et optimisé pour le référencement sur les moteurs de recherche. 


\section{Module introduction}

Ce texte doit donner envie au lecteur de poursuivre son parcours de lecture du dossier, en lui promettant un contenu, en provoquant sa curiosité sur un sujet qui a su attirer son attention. Il commence par répondre à la question du « quoi ? ", en situant l'actualité du sujet exposé et en explicitant la problématique choisie. S’il s'agit d'un travail à plusieurs mains, il est nécessaire de confronter les réflexions individuelles respectives pour dégager une formulation commune de la problématique.

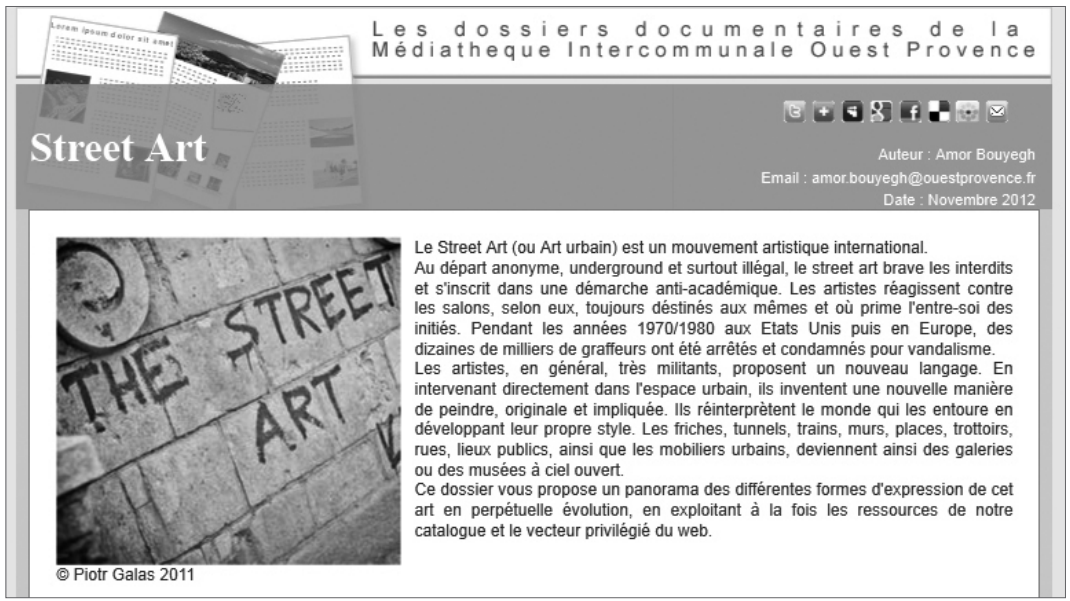

Exemple de l'introduction du dossier "Street art".

\section{Notions clés}
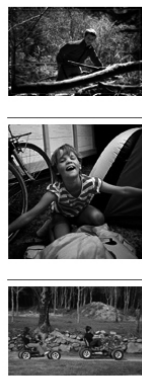

Je prépare mon voyage

Organisation, réservation, précautions. jeunes parents partez l'esprit tranquille en vous connectant sur ce site qui vous sera de bon conseil.

Je choisis ma destination

II n'est pas possible de dresser une liste exhaustive des destinations, cela dépend de lâge de l'enfant, de la saison...quelques pistes de réflexions pour vous aider à définir vos projets voyageenfamille.com

Je me déplace

Partir oul ace mais comment ? Diter moyens de transports sont possibles, cela dépend de votre destination, de l'âge de (a) sans les désagréments et en toute sécurité, c'est possible : en vort

\section{Module Notions clés}

Ce module vise à exposer les idées forces du sujet du dossier, à offrir un aperçu rapide de son cadre d'analyse et de questionnement.

Exemple du dossier :

"Voyager avec ses enfants" 


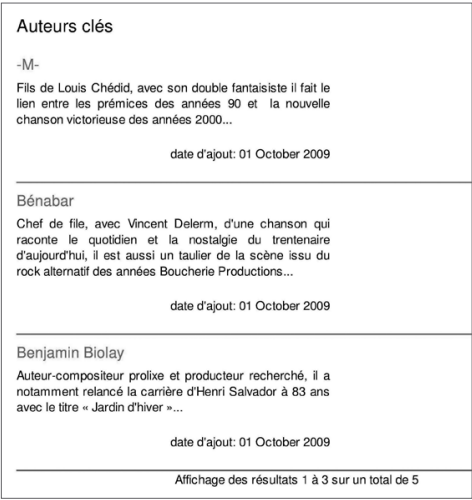

Exemple du dossier :

"La chanson française des années 2000 »

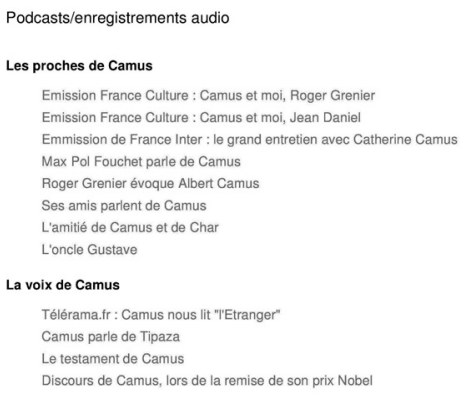

Exemple du dossier :

«Camus l'intime»

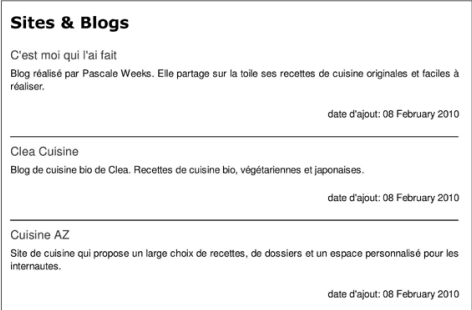

Exemple du dossier :

"Le Web en cuisine »

\section{Module Auteurs clés}

Plus rarement utilisé, ce module peut venir compléter les notions clés. La consigne est de ne pas dépasser 5 références d'auteurs, parmi les plus pertinentes. La mention d'auteur est précisée en en-tête, accompagnée de 3 lignes de texte maximum sur la biographie et l'œuvre de l'auteur.

\section{Modules de médias (images, vidéos, podcasts)}

En fonction de la thématique choisie, ces 3 widgets sont utilisés en tout ou partie, intégrant les médias les plus aisément exploitables par l'utilisateur comme les plus pertinents en termes de complémentarité avec la forme texte. Le widget vidéo peut comporter, au maximum, une vidéo embarquée en lecture directe dans le dossier, les autres étant fournies sous forme d'hyperliens (10 maximum).

\section{Module Sitothèque}

Une sélection des meilleurs sites et blogs (10 maximum) sur le sujet. Chaque sélection comporte un texte court (3 lignes) présentant l'essentiel du contenu du site. 


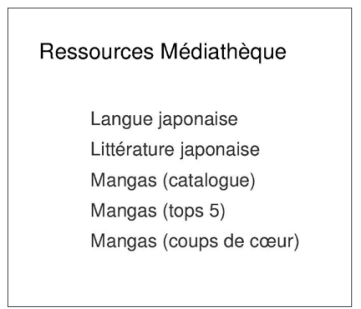

Exemple du dossier : "Il était une fois le Japon »

\section{Module Ressources médiathèque}

Des sélections de documents issus du catalogue sous forme de listes bibliographiques. Elles sont limitées au nombre de 5 (comprenant chacune 10 sélections au maximum) et explicitement nommées, avec une URL* unique et pérenne, au même titre que les documents qu'elles contiennent ; leur contenu peut faire l'objet d'une mise à jour régulière au gré de nouvelles acquisitions.

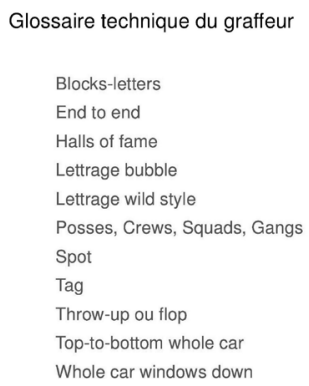

Exemple du dossier "Street art"

\section{Module Lexique-glossaire}

Une définition des concepts essentiels (15 maximum) liés à la problématique. Chaque terme ouvre une fenêtre contextuelle avec la définition correspondante.
Questions-Réponses

Peut-on passer le bac si l'on a un handicap? Quels conseils donner aux parents des bacheliers? Oũ trouver des révisions pour un bac pro ? Comment sont élaborés les sujets du bac? Le TPE fait en 1ère compte t-il pour le baccalauréat?

Exemple du dossier " Réviser son $B A C$ »

\section{Widget Questions-réponses}

Des réponses apportées aux questions les plus fréquemment posées sur le sujet. Chaque question (10 maximum) est un lien qui ouvre une fenêtre contextuelle contenant la réponse correspondante. 


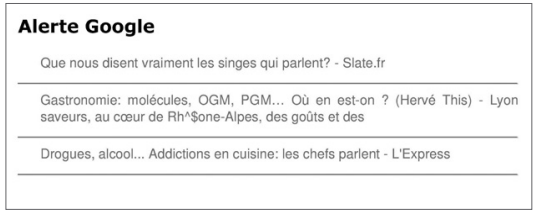

Exemple du dossier : "Des molécules dans nos assiettes »

\section{Pour aller plus loin}

Fédération française des professeurs de français

CIEP : Centre intermational d'études pédagogiques

Phonétique.fr : Apprendre le français avec des exercices de phonétiques

Bonjour de France : Cours de FLE en ligne

Polar du FLE : Apprendre et étudier le français avec linspecteur Duflair

Fiépertoire des méthodes de FLE

Français langue ótrangère - FLE : site très complet pour découvrir et apprendre le FLE

Exemple du dossier «FLE»

\section{Module Alerte Google}

Des fils RSS (4 alertes maximum) qui offrent une veille continue sur le sujet en actualisant automatiquement leur contenu.

\section{Module Pour aller plus loin}

Un renvoi (au nombre de 10 maximum) vers des ressources complémentaires permettant d'approfondir et/ou d'élargir le sujet du dossier.

\section{ENCADRÉ DOSSIER DOCUMENTAIRE : LES ÉTAPES DE PRODUCTION}

1. rédaction de la fiche-projet, autour de ces problématiques principales: quel est le sujet? Pourquoi ce sujet? Qui portera le projet ? Pour qui ? Quand?

2. recherche-collecte d'informations : ce travail d'exploration ou de curation s'effectue principalement sur le Web.

3. extraction-sélection des informations les plus utiles au regard du sujet et du cadre de synthèse préalablement établis.
4. structuration des informations : cette étape consiste à déterminer quels sont les blocs ou widgets à exploiter pour agencer au mieux les documents et médias sélectionnés.

5. rédaction Web : la pratique d'écriture est mobilisée sur la partie introductive du dossier ainsi que sur les notions clés.

6. indexation : métadonnées (tags et catégories).

7. dissémination : les conditions de diffusion et d'audience du produit éditorial... 


\section{COMMENT PRODUIRE UN DOSSIER : QUELLES ÉTAPES ? QUELS OUTILS ?}

La question de l'archivage est également anticipée dès la phase de conception du projet. Si la pérennité du dossier est admise, les conditions de sa mise à jour sont précisées. L'auteur du produit vérifie régulièrement l'obsolescence des données exploitées ainsi que les liens brisés.

\section{À QUELS PUBLICS S' ADRESSENT LES DOSSIERS ?}

Le niveau de lecture requis d'un dossier varie en fonction du public visé, mais sa fonction d'outil en bibliothèque publique lui confère $a$ priori un niveau « moyen » adapté au grand public.

Malgré cette absence de cible désignée sur la majorité des dossiers produits par la MIOP, le projet doit néanmoins interroger les éléments suivants :

- de quoi l'utilisateur peut-il avoir « besoin » sur ce sujet ?

- que vient-il chercher en s'intéressant à ce sujet?

- quelle est la valeur réelle de l'information délivrée ?

- quelle est la valeur d'usage du contenu ?

- comment dois-je présenter l'information pour susciter au mieux son intérêt, capter son attention?

Dans le cadre d'une co-construction réalisée avec les partenaires de l'éducation, enseignants des collèges et lycées, le dossier sera plus conforme à son statut dit de " produit ", un document répondant à une commande précise explicitement formulée par son demandeur².

2. Deux exemples : «La permaculture », dossier réalisé avec une classe de seconde du lycée Fontlongue de Miramas < http://www.mediathequeouestprovence.fr/index.php?id=2970 > et « Quelle agriculture pour demain ? », dossier réalisé avec la contribution des élèves de $5^{\mathrm{e}}$ du collège A. Malraux de Fos-sur-Mer < http://www.mediathequeouestprovence.fr/index.php?id=2843 >. 


\section{QUI PRODUIT LES DOSSIERS DOCUMENTAIRES ?}

À la MIOP, la production des différents types de contenus numériques et leur médiation sont une activité professionnelle inscrite dans la fiche de poste de l'agent, notamment de celui qui exerce une responsabilité documentaire (soit 50 agents). Dans le cadre de cette activité, le sujet du dossier est logiquement corrélé au domaine de contenu dont l'agent a la charge, ce dernier étant nécessairement un spécialiste (non un expert) du champ disciplinaire principal couvert par la thématique du dossier.

Conduit en général par son seul initiateur, le dossier peut gagner en complétude s'il est réalisé à plusieurs mains. On évite néanmoins de dépasser trois contributeurs sur un même projet et on veille à bien répartir le travail d'élaboration du dossier.

En termes de compétences, la réalisation d'un dossier requiert des savoirfaire particuliers, propres à l'environnement Web. Une formation à l'écriture Web a été dispensée à cette fin en intra, auprès de l'ensemble des responsables documentaires. Cette formation a permis de mesurer plus exactement les compétences nécessaires à toute pratique de production et de médiation de contenus numériques :

- savoir cerner le sujet, énoncer une problématique, formuler un questionnement ;

- maîtriser les outils de veille et techniques de recherche documentaire ;

- savoir collecter les données afin d'établir un corpus informationnel ;

- savoir choisir des contenus d'information et de connaissance en fonction de leur pertinence ;

- savoir définir le niveau d'information ;

- savoir organiser ces sélections en les structurant, en les hiérarchisant, en les mettant en relation logique ;

- maîtriser les techniques rédactionnelles propres au Web ; 
- maîtriser l'outil de gestion et de publication de contenus utilisé par l'établissement ;

- savoir disséminer le document produit sur la toile, favoriser sa visibilité sur d'autres plates-formes de diffusion.

La mise en place d'une chaîne éditoriale efficace, encadrant les contributions individuelles, ainsi que le choix d'outils de publication souples et puissants participent de la réussite et de la viabilité du projet à long terme. À la MIOP, le chef de département est un acteur fort de ce dispositif, assumant une fonction tout à la fois managériale et scientifique du projet, depuis sa validation formelle jusqu'à son évaluation.

Le directeur de la politique documentaire procède aussi, en bout de chaîne de publication, à une lecture complète du dossier, en considérant la valeur didactique de l'ensemble du document ainsi que sa conformité à la ligne éditoriale prescrite pour ce type de produit.

\section{QUELLE ÉVALUATION POUR LES DOSSIERS ?}

Au-delà du simple compteur de visites, il est difficile de mesurer le taux de satisfaction d'un dossier diffusé collectivement, à la différence des dossiers " produits ", visant un public donné qui peut être interrogé au moyen d'un questionnaire.

On peut également regretter que la structuration du dossier numérique par module ne permette pas une évaluation différenciée de chacun de ces objets.

Le nombre de visites est néanmoins un critère d'activité intéressant, permettant d'évaluer le niveau de performance des dossiers qui font l'objet d'une mise à jour régulière et qui génèrent un coût cognitif et de gestion important.

À la MIOP, le premier dossier a été réalisé fin 2008 et le portail en compte désormais, en ce début 2014, près de 50. L'outil d'analyse d'audience Google Analytics permet d'apprécier une consultation en hausse constante de ces différentes productions, les dossiers les plus visités dépassant à ce jour les 3000 visites. 


\section{UN SERVICE À PART ENTIÈRE QUI ÉVOLUE}

Au commencement de notre exposé, nous avons considéré, l'importance grandissante du besoin d'informations synthétiques dans notre société et son expression manifeste dans des contextes et situations divers. Plus le morcellement et la dispersion des savoirs feront en effet partie de notre univers, plus nous devrons faire appel à des structures cognitives et outils de gestion informationnelle pour gérer cet éclatement. C'est là l'objectif premier de ce produit à haute valeur ajoutée que constitue le dossier documentaire numérique, une forme de carte mentale qui insère, combine, agrège, structure, unifie des éléments fragmentés et hétérogènes et, facilite la compréhension de ce formidable gisement de connaissances disponible en flux continu sur la toile.

C'est aussi pour les professionnels des bibliothèques un exercice particulièrement formateur, dans ses phases de conception comme de fabrication, un exercice mobilisant des compétences intellectuelles et méthodologiques au cœur de leur métier.

À la MIOP, la qualité et la vitalité de ce travail résultent de la cohérence et de l'efficience de l'organisation mise en place depuis plus de dix ans au sein de la direction de la politique documentaire ; une organisation qui repose avant tout sur le dynamisme des agents en charge des collections du réseau, ces 50 chevilles ouvrières de la sélection, de la production, de la gestion et de la médiation des contenus.

La politique de formation à destination de ces contributeurs, ainsi que le choix stratégique de l'outil de publication, dans ses fonctionnalités d'usage et ses potentialités d'évolution, ne sauraient pour autant être négligés, car ils conditionnent aussi la réussite et la pérennité du projet.

Notons que d'autres projets sont actuellement en cours de finalisation à la MIOP : les « p'tits docs », (dossiers à destination des enfants) et les «WebZooms ", (une synthèse documentaire plus légère, produite à la demande). Le dossier numérique est ainsi devenu, au même titre que la collection, un service à part entière, accessible au plus grand nombre, un service qui relie des savoirs comme des compétences et qui concourt à l'élargissement de la sphère informationnelle de la bibliothèque. 
On pourrait alors espérer la construction d'une plate-forme commune à l'échelle nationale, permettant de fédérer en un point d'accès unique l'ensemble des dossiers documentaires aujourd'hui diffusés et répartis sur les différents portails des bibliothèques et centres de documentation. Pour les professionnels de ces structures, une telle base de ressources permettrait de mettre en regard et en synergie ces différents objets documentaires, chacun produits avec des moyens humains, techniques et organisationnels particuliers. Gageons que la publication de ce nouvel opus de la Boîte à outils puisse y concourir. 


\section{2}

LES AGRÉGATEURS DE CONTENUS ET LA CURATION À LA MÉDIATHĖQUE DE BAGNOLET

par

Dominique Macé

\section{LE TEMPS DES EXPÉRIENCES : 2008-2009}

Fin 2008, lorsque la médiathèque de Bagnolet accueille la résidence de François Bon, le personnel est loin d'imaginer les suites de ce qui semble alors une expérience limitée dans le temps. François Bon affirme très clairement son projet : "Aujourd'hui, tout va plus vite, et prend de nouvelles formes : ainsi le rôle de l'ordinateur. » et il ajoute : "C'est sous ce signe que je voudrais placer cette résidence. »

C'est en effet une période charnière où le Web n'est pas encore entré massivement dans les pratiques quotidiennes. Les matériels nomades sont des objets réservés aux geeks. Les ressources numériques sont rares.

$\mathrm{Au}$ cours de cette résidence, la médiathèque ouvre en 2009 un site Web ${ }^{1}$ avec un logiciel libre Spip installé par un prestataire. Deux de nos collègues, déjà familiarisés avec la publication sur le Web par une pratique personnelle, deviennent administrateurs de ce site officiel validé par l'autorité de tutelle.

Dès lors, la résidence de François Bon va accélérer l'initiation puis la pratique des outils Web pour une bonne partie du personnel de la médiathèque. Un atelier Web est spécialement réservé aux professionnels des bibliothèques et médiathèques de la région. La médiathèque de Bagnolet est équipée d'ordinateurs avec accès Internet pour le public. La connexion Internet n'est pas idéale et le matériel n'est pas des plus récents. Au départ, peu nombreux sont ceux, parmi les membres du personnel, qui connaissent les outils du Web. Plus qu'une formation technique, c'est l'occasion d'expérimenter des formes de travail entièrement nouvelles.

1. < http://mediatheque.ville-bagnolet.fr >. 


\section{UN TRAVAIL COLLABORATIF}

Le site de la médiathèque, sous Spip, permet un fonctionnement collectif : tous les collègues, quelle que soit leur fonction ou leur grade, peuvent rédiger un article, publier une bibliographie.

L'organisation des rubriques sur le site reflète l'organisation des collections matérielles.

Les fonds de la médiathèque sont organisés en quatre départements : Documentaires, Littérature-Langues-BD, Musique et Jeunesse.

Chaque département prend en charge tous les supports (imprimés, son, vidéo) pour les acquisitions, le traitement des documents et l'action culturelle (médiation). Il en va de même pour le site : chaque département a en charge sa rubrique et doit l'alimenter régulièrement en publiant des articles.

Le site fonctionne comme un blog, chaque article publié est en vedette jusqu'à la publication d'un autre article.

L'organigramme de la médiathèque n'ayant pas de département " multimédia » ou « Web », nous avons organisé dans un premier temps des réunions de coordination qui faisaient office d'initiation à la pratique de l'interface. Mais très vite, cette forme de travail s'est avérée inadaptée aux exigences de temps et de planning. Nous avons donc opté pour un travail collaboratif : dans chaque département il y a au moins un collègue chargé de publier sur le site.

Les contenus publiés sur le site sont en lien avec le travail régulier de chaque département : il s'agit toujours de la mise en valeur des fonds à l'occasion d'une actualité, d'un événement auquel la médiathèque fait écho. La coordination, la veille sur le respect des règles de publication sur le plan juridique, les corrections éventuelles sont assurées par le collègue administrateur du site qui a aussi mis en place des mini-formations. Le renouvellement des contenus a son importance pour la fidélisation des internautes. Le travail collaboratif permet une souplesse de fonctionnement qui favorise l'envie de publier sur le site. 


\section{LES PRODUCTIONS DE LA MÉDIATHĖQUE}

La médiathèque de Bagnolet publie régulièrement des bibliographies, des filmographies, des discographies pour mettre en valeur les acquisitions récentes (nouveautés) ou des choix thématiques à l'occasion de différents événements (expositions, concerts, rencontres diverses, actualités, etc.). Ces publications sont d'abord réalisées à partir d'un tableau sur traitement de texte où sont intégrées les données des notices bibliographiques. Puis le tableau est transféré sur un modèle de mise en page qui sera édité sous le format PDF. Les PDF sont ensuite envoyés sur Calaméo, le site de publication en ligne sur lequel la médiathèque s'est inscrite gratuitement ${ }^{2}$. La publication est ensuite intégrée au site de la médiathèque à l'aide d'une balise (code) générée par le site Calaméo. Chaque département peut ainsi proposer régulièrement une liste de nouveautés, une bibliographie thématique. L'usager pourra visualiser directement sur le site de la médiathèque un petit livret virtuel mais il pourra aussi télécharger le document en format PDF. Cette forme de publication permet aussi la mise en valeur des productions d'ateliers d'écriture.

\section{LES AGRÉGATEURS DE CONTENUS}

En plus de ses propres publications, le site de la médiathèque intègre des outils Web dont la finalité est de faire entrer une sélection numérique produite bien au-delà des murs de la médiathèque. La médiathèque productrice de contenus peut, à son tour, à l'aide de ces outils, diffuser ses publications, les faire vivre sur la toile au-delà du cercle des usagers inscrits. La médiathèque est inscrite sur Netvibes ${ }^{3}$ et sur Pearltrees ${ }^{4}$, deux agrégateurs de contenus qui apparaissent en lien sur le site. Scoop.it, un autre agrégateur, est un outil de veille intégré sous forme de widget. Ces outils sont gratuits. À l'exception de Scoop.it, les interfaces sont en français.

\footnotetext{
2. < http://fr.calameo.com/accounts/25681 >.

3. < http://www.netvibes.com/mediabag93 >.

4. < http://www.pearltrees.com/media_bagnolet >.
} 


\section{ENCADRÉ NETVIBES, PEARLTREES, SCOOP.IT}

- Netvibes est un portail personnalisable qui permet d'afficher : des flux RSS, des liens, des pages Web, sous différentes formes : textes, sons, vidéo. Deux types d'affichage sont proposés, mais c'est l'affichage par widget qui demeure le plus agréable à consulter ; l'organisation par onglet permet de créer des rubriques thématiques, un widget permet de faire des annotations à l'aide d'un éditeur HTML. L'affichage des flux RSS, des liens sur les sites, des pages Web est aussi personnalisable. La diversité des modes d'affichage des contenus donne au Netvibes l'aspect d'un présentoir ou d'une table comme celle que l'on utilise dans la médiathèque "physique ».

- Pearltrees agrège sous forme de « perles » des contenus produits par la médiathèque, pages Web, textes sur Calaméo, albums photo, vidéos. Les perles peuvent être rassemblées dans une perle thématique à laquelle peuvent s'amarrer d'autres perles thématiques disposées en branches. L'ensemble finit par former un arbre. Cet agrégateur donne une image globale des productions et sélections de la médiathèque. Le bouton Voisin donne accès à d'autres " perles » venues d'ailleurs dont les thématiques se rejoignent. L'arborescence est alors potentiellement infinie.

- Scoop.it fonctionne un peu comme un micro-blogging très visuel. On capture l'adresse d'une page Web puis on publie sur Scoop.it où elle apparaît en lien. Scoop.it intègre aussi une image d'en-tête et les premiers mots du texte de la page sélectionnée. On peut éditer un commentaire, une présentation personnalisée. Les sélections s'affichent à la manière d'un journal mural que l'on peut lire en déroulé vertical.

Les agrégateurs de contenus installés sur le site ouvrent sur une bibliothèque virtuelle.

C'est un nouveau " rayonnage » qu'il faut alimenter régulièrement. L'indexation et le catalogage ne sont plus nécessaires. Le bibliothécaire choisit des textes, des vidéos, des sons, dans l'immense collection immatérielle que constitue le Web. La mise en valeur qui permettra aux internautes de trouver ces contenus consiste à donner du sens en créant des thèmes, en insérant des commentaires, en faisant un lien avec les collections physiques. 


\section{LA LITTÉRATURE SUR LES TABLES DU WEB}

\section{Rendre visibles des flux numériques}

La littérature, ses débats, son actualité, est présente sur le Web comme dans le monde réel. Pour autant, comme dans le monde réel, elle ne fait pas partie des préoccupations majoritaires. Défi d'autant plus grand pour les bibliothécaires du monde virtuel. Mettre en valeur des textes, des expressions artistiques hybrides émergeant avec ces technologies, n'est pas si différent de ce que nous faisons avec les livres, les DVD, les CD, les revues. Cependant, l'environnement numérique ne ressemble en rien à ce que nous connaissons lorsque nous faisons des acquisitions de documents matériels. Il ne s'agit plus d'enrichir une collection mais de rendre visibles des flux numériques.

\section{utilisation du Netvibes}

Contrairement au site de la médiathèque alimenté par des contributions de tous les départements, le Netvibes est, pour l'instant, essentiellement consacré à la littérature, la BD, et l'actualité artistique.

L'une des rubriques, "écrivains du Web », donne accès aux flux RSS des blogs d'auteurs dont un certain nombre publie aussi des livres imprimés ou numériques. La rubrique " la bouquinosphère " présente les blogs de la critique littéraire et artistique sur Internet.

Cette sélection est à la disposition des internautes, mais c'est aussi un outil professionnel devenu indispensable puisqu'elle donne accès à l'atelier de l'écrivain autant qu'à ses liens avec le Web littéraire.

Ce ne sont plus des œuvres encloses dans un livre que l'on découvre en entrant dans ces ateliers d'écriture mais un travail de recherche fait de tâtonnements, d'essais, de fragments. Et parfois, à l'occasion d'une visite en librairie, le plaisir de retrouver le livre imprimé rassemblant et ordonnant le travail du blog.

\section{utilisation du scoop.it}

Nous avons installé sur le site de la médiathèque deux widgets Scoop.it. Ces Scoop.it sont thématiques : 
- Des polars à Bagnolet ${ }^{5}$ est une sélection d'articles critiques sur des œuvres que les lecteurs peuvent trouver sur les rayonnages de la médiathèque. Une revue de presse en quelque sorte. Scoop.it permet de taguer les sélections. On peut classer, grâce aux tags, les articles sélectionnés et proposer des entrées multiples, polars nordiques, polars historiques par exemple, entrées qui n'existent pas dans les critères de recherche du catalogue de la médiathèque ;

- La bibliothèque hors le livre ${ }^{6}$ attire l'attention sur des productions numériques littéraires et artistiques présentées sous formes de textes, de vidéos, d'enregistrements sonores. En littérature, l'actualité sur le Web n'est pas celle de la production imprimée C'est tout l'intérêt d'aller chercher sur la toile des textes, des expressions artistiques absents des rayonnages. Le hasard de la navigation par les liens peut mener à toutes sortes de découvertes auxquelles ni les bibliothécaires ni les lecteurs n'auraient pensé. Présentée comme " signets », cette sélection n'a rien d'exhaustive ou d'exclusive. Pour la plupart, ces productions numériques n'ont pas d'existence matérielle. Les textes repérés dans les flux de Netvibes ont été capturés sur des blogs d'écrivains ou en cliquant sur les liens des blogs amis ;

- d'autres formes de productions littéraires et artistiques peuvent être mises en valeur par un Scoop.it : une lecture performance en vidéo sur une revue en ligne, un podcast d'émissions littéraires radiophoniques, une interview filmée sur le site d'un éditeur.

Le monde de l'imprimé n'est pas exclu pour autant. Le Web littéraire est truffé de références au patrimoine imprimé. Si la découverte de la littérature passe de plus en plus par le Web, le livre comme support est encore un moyen privilégié d'accéder à ce patrimoine.

5. < http://www.scoop.it/t/des-polars-a-bagnolet >.

6. < http://www.scoop.it/t/la-bibliotheque-hors-le-livre >. 
Les revues ont une place importante dans le monde littéraire. La migration numérique est en marche. Les revues littéraires adoptent de plus en plus une double publication, sur papier et sur le Web. Publier sur Scoop.it les pages présentant les revues auxquelles la médiathèque s'est abonnée, le sommaire du dernier numéro par exemple, augmente sans aucun doute les potentialités d'emprunt.

\section{partager sur les réseaux sociaux}

Tous ces agrégateurs ont des fonctions de partage sur les réseaux sociaux. La médiathèque peut ainsi propulser les sélections numériques collectées sur les agrégateurs de contenus. La visibilité des sélections mais aussi du site s'en trouve renforcée.

Le site Calaméo est bien référencé par les moteurs de recherche. Les statistiques du site font apparaître une fidélisation du lectorat pour les listes en ligne au format Calaméo. Les usagers peuvent à tout moment, et surtout en n'importe quel lieu, consulter ces listes, vérifier s'ils trouveront le livre qu'ils convoitent sur les rayons de leur médiathèque.

Il suffit d'un clic pour transférer le journal Scoop.it, les Pearltrees, sur le Facebook de la médiathèque ${ }^{7}$ ou en lien sur Twitter. Les tables du Web se présentent alors instantanément devant l'usager connecté à ce moment-là. Utiliser les fonctions de partage, c'est aussi s'abonner aux Netvibes, Scoop.it, d'autres médiathèques ou de « veilleurs » du Web. On reçoit alors des sélections qui enrichissent les thématiques proposées par sa médiathèque.

\section{ATTENTES DES USAGERS ET NOUVELLES PRATIQUES PROFESSIONNELLES}

Même si le travail collaboratif incite l'ensemble du personnel de la médiathèque à publier des contenus sur le site, seule une partie des personnels contribue à enrichir la bibliothèque virtuelle.

7. < https://www.facebook.com/mediatheque.bagnolet >. 


\section{LA CURATION}

La publication sur le site des listes de nouveautés est devenue une priorité. En effet, les adhérents de la médiathèque attendent ces publications comme un service indispensable. L'accès quasi général aujourd'hui aux appareils nomades connectés n'y est pas pour rien.

En revanche, la curation de contenus* n'est pas une pratique professionnelle majoritaire parmi les membres du personnel. Elle est encore une démarche volontariste. Mais il est vrai que cette pratique de curation est encore un peu expérimentale pour nous. Elle bouleverse les habitudes.

Le professionnel est confronté à l'incommensurabilité du Web. À l'inverse de la collection physique présente sur les rayonnages en permanence, la collection virtuelle est fluide, parfois éphémère. La hiérarchisation et la validation par des autorités intellectuelles ne sont pas toujours perceptibles.

La curation exige une veille régulière et une mobilisation de la mémoire, de l'attention, et donc une organisation du travail qui prenne en compte ces nouvelles pratiques. À la médiathèque de Bagnolet, le choix d'organiser le travail à l'image de l'organisation des collections, c'est-à-dire sur les contenus avant tout, permet d'évoluer vers une implication plus large du personnel pour étendre la pratique de la curation aux sciences humaines, au domaine scientifique.

\section{NOUVEAUX PUBLICS}

Il ne fait aucun doute que la curation d'agrégateurs de contenus par la médiathèque attire de nouveaux publics et contribue à la mise en réseau de petites communautés d'intérêts.

La curation comme pratique professionnelle ne consiste pas uniquement à prescrire. Elle ouvre sur des univers ignorés des médias traditionnels, elle attise la curiosité, finalement, elle contribue de plus en plus à l'enrichissement des fonds physiques. Le va-et-vient entre la bibliothèque virtuelle et la bibliothèque physique préfigure sans doute de nouvelles formes de travail qu'il faudra bien un jour généraliser. 


\section{3}

NUMÉRISATION DE MASSE, MÉTADONNÉES ET BIBLIOTHÈQUE NUMÉRIQUE : L'EXEMPLE DE NUMELYO À LA BIBLIOTHĖQUE MUNICIPALE DE LYON

Lorsqu'en 2008 la ville de Lyon annonce la signature d'un contrat de numérisation avec Google, pour 500000 livres imprimés, la Bibliothèque municipale de Lyon $(\mathrm{BmL})$ s'appuie sur une solide expérience de la numérisation. Depuis 1993, elle a conduit plusieurs projets de numérisation en appliquant les choix techniques de l'époque : numérisation en mode image et mise en valeur via des outils de gestion électronique de documents (GED) qui permettaient la diffusion des images sur Internet associée à quelques services (rechercher des documents via des métadonnées peu normalisées, afficher l'image et l'agrandir, imprimer et télécharger l'image).

En 2008 donc, la bibliothèque proposait déjà sept bases de données (BDD) patrimoniales sur son site $\mathrm{Web}^{1}$, développées via un outil de GED, chaque base étant indépendante l'une de l'autre ${ }^{2}$. Par exemple, chacune disposait de son propre set de métadonnées peu normalisées.

L'enrichissement de chaque base avait nécessité un travail titanesque puisque chaque image donnait lieu à la création ex-nihilo d'une notice via l'outil de GED. Pourtant, ces bases de données patiemment constituées ne rencontraient pas le succès escompté. Peu d'internautes venaient s'aventurer dans les collections numérisées, sinon armés de solides connaissances sur les fonds disponibles et sur les langages d'indexation.

Enfin, si depuis 1993, la numérisation s'était largement portée sur les œuvres iconographiques et manuscrites, se posait de façon criante la question de la numérisation et de la diffusion des collections patrimoniales

1. Enluminures, affiches, estampes, provenances des livres anciens, documents issus de l'école des textiles de Lyon et manuscrits Carolingiens, ainsi qu'une base de dossiers de presse dont le contenu, non libre de droit, n’était consultable que dans l'enceinte de la bibliothèque.

2. À partir de 2003, la mise en place de Catalog+, moteur de recherche fédéré, a rendu possible l'interrogation simultanée des BDD. 
imprimées. Si le patrimoine que conserve la bibliothèque comprend de nombreuses œuvres uniques, il est aussi largement constitué de collections d'imprimés. Comment pouvait-on ne pas s'interroger sur la mise en valeur de ces collections considérables qui constituent le cœur des collections patrimoniales ? L'extension du plan de numérisation aux collections d'imprimés constituait donc un enjeu fondamental pour la bibliothèque. Il s'agissait alors pour la BmL, d'une part, d'étendre son plan de numérisation aux collections massives d'imprimés et, d'autre part, de penser la mise en valeur de toutes les collections numérisées via une bibliothèque numérique.

\section{PENSER LA BIBLIOTHĖQUE NUMÉRIQUE ET ÉTENDRE LE PLAN DE NUMÉRISATION AUX COLLECTIONS IMPRIMÉES}

Si la réflexion de la $\mathrm{BmL}$ sur la bibliothèque numérique faisait écho aux limites de l'offre qu'elle proposait à l'époque, elle s'inscrivait aussi dans un contexte plus large. Par exemple, comment concevoir une bibliothèque numérique dans une nouvelle cartographie du Web ? En effet, en 2004 lorsque Google lance Google Print, rebaptisé en 2005 Google Book Search, il devient manifeste que les bibliothèques ne sont plus les seules pourvoyeuses de collections patrimoniales sur Internet. Elles ne sont pas non plus les plus utilisées. Que devient alors la place d'une bibliothèque numérique et des bibliothécaires lorsque la plus grande bibliothèque numérique n'est pas une bibliothèque ? Que devient la spécificité d'une bibliothèque numérique produite par une bibliothèque ?

Outre cette question cartographique, se posait également la question des usages du Web dans un contexte où les pratiques et les attentes étaient en évolution avec le Web 2.0 : quelle devait ou pouvait être la forme d'une bibliothèque numérique tenant compte de ces évolutions d'usages? Même s'il ne s'agit pas du seul élément de réponse, on peut se souvenir que la création des services de référence avait montré que non seulement le bibliothécaire avait un rôle à jouer dans la mise à disposition de contenus mais qu'il pouvait également devenir créateur de contenus. Cette création de contenus éditorialisés par les bibliothécaires au sein d'une bibliothèque 
numérique - et plus généralement du site Web de la bibliothèque - initiait une réflexion importante.

\section{LE CONTEXTE TECHNIQUE}

Le cadre s'était aussi enrichi d'un point de vue technique, grâce à une nouvelle offre logicielle notamment dans le domaine des logiciels libres (Greenstone, Fedora*).

Du point de vue des métadonnées, la maîtrise d'autres langages descriptifs que le MARC se diffusait au sein de la communauté des bibliothèques. Par exemple, plusieurs bibliothèques avaient acquis une connaissance et une expérience du XML via notamment l'encodage en Encoded Archival Description (EAD) d'inventaires d'archives. Cette première approche du XML pouvait ouvrir, au sein des bibliothèques concernées, une réflexion concrète et sereine sur d'autres façons d'aborder les métadonnées que le catalogage en Unimarc.

\section{DES OPPORTUNITÉS DE FINANCEMENTS À TROUVER}

S'il était indispensable de repenser la bibliothèque numérique, il fallait également envisager l'enrichissement des collections numériques. Si l'importance d'élargir la numérisation aux collections imprimées était partagée, la question du financement de ces opérations demeurait problématique, même si BmL avait pu bénéficier à cette époque d'aides de la part du ministère de la Culture pour la numérisation de certains fonds patrimoniaux. Le recours à des partenariats publics-privés (PPP) fournissait une option qu'il était raisonnable de prendre en compte. C'est dans ces conditions que la ville de Lyon a décidé de lancer un appel d'offres pour la numérisation gratuite de 500000 livres imprimés libres de droit.

\section{L'ARTICULATION DE DEUX PROJETS}

Le 18 juillet 2008, la ville de Lyon signe donc un contrat avec Google pour la numérisation de 500000 livres imprimés libres de droits, marquant ainsi une étape décisive pour l'extension du plan de numérisation de la bibliothèque et pour la création d'une nouvelle bibliothèque numérique, Numelyo, inaugurée le 12 décembre 2012. 
La numérisation de masse et la création de Numelyo sont deux projets qu'il est difficile de séparer. D'une certaine façon, c'est parce que la bibliothèque voulait mettre en place une importante bibliothèque numérique qu'il lui fallait atteindre une véritable masse critique de documents mais c'est aussi parce qu'avec le projet Google elle a eu l'opportunité de disposer d'une masse importante de documents numérisés, qu'il lui fallait une véritable bibliothèque numérique.

Cette bibliothèque numérique, la bibliothèque fait alors le choix de la développer en interne. Le projet comprenait donc de multiples volets et donc de nombreux problèmes à résoudre :

- intégrer dans un outil unique, à la fois les collections qui avaient été précédemment numérisées, les 500000 imprimés numérisés prévus dans le cadre de la numérisation de masse et les documents numérisés dans le cadre de projets en cours ou ultérieurs ;

- proposer dans la future bibliothèque numérique des contenus produits par les bibliothécaires contextualisant les collections : expositions virtuelles, dossiers, collections, articles... conférant ainsi à cette future bibliothèque numérique une spécificité ;

- développer des outils susceptibles de gérer efficacement des masses importantes de documents et capables d'intégrer de nouveaux services ainsi que des contenus éditorialisés autres que des métadonnées. Ces mêmes outils devaient être évolutifs et rendre possible le partage et l'archivage des données.

Le choix s'est donc porté sur la plate-forme libre de bibliothèque numérique Fedora. Quant aux métadonnées, Numelyo utilise des métadonnées METS* (format d'encodage), pour les métadonnées de conservation encapsulant des métadonnées descriptives en MODS* (schéma XML pour la description bibliographique de contenus). Le choix d'utiliser Fedora n'aurait pu être possible si la bibliothèque n'avait pas compté dans ses effectifs un ingénieur développeur. 


\section{LE CONTRAT SIGNÉ AVEC GOOGLE : MASSE CRITIQUE ET NUMÉRISATION DE MASSE}

Le contrat signé le 18 juillet 2008 explicitait l'objet de la prestation et ses conditions, conformément à l'appel d'offres lancé par la ville de Lyon, Google s'engageait à numériser entre 450000 et 500000 livres imprimés et libres de droits, dont 200000 dans un délai de 4 ans ainsi qu'à les mettre en ligne à titre gratuit sur Google Books.

\section{ENCADRÉ 1 LE CADRE DU CONTRAT GOOGLE}

- sont à la charge de Google : le transport des documents, leur numérisation effectuée dans un rayon inférieur à $50 \mathrm{~km}$ autour de la bibliothèque impliquant donc la construction d'un centre de numérisation par Google au sein de ce périmètre, la numérisation produite, donc, en mode image et en mode texte ; la mise en ligne des documents via Google Books, la mise à disposition par la BmL d'une interface logicielle lui permettant de télécharger les versions numériques ainsi produites et de les utiliser ;

- conformément au Code des marchés publics, le contrat prévoit que la biblio- thèque ne demandera pas à un autre prestataire de numériser les fonds en question pendant la durée du marché ;

- Google conserve la propriété des fichiers numériques ;

- la BmL s'engage, quant à elle, à fournir les livres à numériser ainsi que les métadonnées les décrivant. En contrepartie, elle obtient une copie de chaque livre numérisé et le droit de les mettre en ligne via son propre site ou de les partager à titre gratuit avec des partenaires à des fins d'étude ou de recherche.

\section{CHAÎNES OPÉRATOIRES ET ORGANISATION DU TRAVAIL}

Les équipes de Google, en 2008, avaient mené plusieurs projets auprès de grandes bibliothèques, notamment européennes, et avaient pris l'habitude de s'adapter aux contraintes et particularités de leurs partenaires. Plusieurs points concernant les documents à numériser étaient précisément définis : 
- être édités avant $1869^{3}$;

- présenter un état matériel défini par des critères stricts ;

- être catalogués au format converti MARCXML ;

- être identifiés par un numéro unique (un code-barres). Les documents patrimoniaux de la bibliothèque ne disposant pas de code-barres, il a fallu générer des codes-barres virtuels.

La bibliothèque a donc conçu un circuit pour gérer les trains de numérisation mais également la circulation des métadonnées. Un logiciel spécifique interfacé avec le logiciel de Google a été développé par la bibliothèque. Il permet de suivre les documents à chaque étape de la numérisation, de télécharger les documents numérisés en attendant un versement dans Numelyo et de convertir puis envoyer à Google les notices en MARCXML. La « matière première » est constituée par les notices bibliographiques du catalogue. Une première extraction des notices de livres antérieurs à 1869 a été effectuée au début du projet. Transformées en MARC21, elles ont été ensuite converties en MARCXML puis versées dans le logiciel de la BmL. Ne sont donc présentes dans le logiciel que les notices des monographies antérieures à 1869. Les ajouts et corrections de notices sont gérés par des extractions journalières du catalogue vers le logiciel. Ce sont ces notices qui sont envoyées au rythme des numérisations à Google. Les notices corrigées de documents qui ont déjà été numérisés sont envoyées par lot à Google tous les trimestres. Charge ensuite à Google de substituer les notices corrigées à celles initialement envoyées dans Google Books.

3. Ce critère défini par Google devait garantir que le document était entré dans le domaine public. 


\section{LES ÉTAPES DE LA CHAÎNE OPÉRATOIRE}

\section{ENCADRÉ 2 POUR LA NUMÉRISATION}

- sélection des documents selon des critères définis de date, d'état matériel, de présence d'une notice bibliographique ;

- préparation des trains de numérisation ;

- numérisation effectuée par Google ;

- retour de documents ;
- contrôle de la numérisation ;

- injection dans la bibliothèque numérique. Cette phase n'est intervenue que deux ans après l'envoi du premier document numérisé par Google, alors que Numelyo avait atteint un niveau fonctionnel de développement.

\section{Les équipes}

Deux équipes mettent en œuvre ce circuit : une première équipe constituée de 7 personnes sélectionne les documents, prépare les trains de numérisation et range les documents au retour. Une seconde équipe de 8 personnes contrôle la numérisation et les métadonnées. Au sein de cette équipe, deux personnes complètent, en amont de la numérisation, les métadonnées parcellaires des recueils factices : ceux-ci ayant fait l'objet d'une chaîne opératoire particulière, leur traitement bibliographique se révèle inadapté aux besoins de la numérisation.

Si les grandes phases de cette chaîne opératoire se retrouvent dans tout projet de numérisation, le caractère massif de cette numérisation induit toutefois un changement méthodologique important pour la phase de sélection qui relève alors davantage d'un acte procédural clairement défini ou d'une évaluation sur critères que d'un choix puisqu'il ne préjuge pas les usages qui seront faits des documents numérisés.

\section{NUMELYO ET LES MÉTADONNÉES}

La première question que posait le développement de Numelyo était l'intégration de sources et de métadonnées hétéroclites : 
- des bases de données en mode image accompagnées de métadonnées «maison »;

- des documents numérisés en modes image et texte issus de Google comprenant des métadonnées produites nativement en Unimarc ;

- des documents numérisés en mode image et texte (Alto) issus des numérisations de périodiques en cours comprenant des métadonnées en MODS issues de conversions de notices en Unimarc.

Forte de l'expérience accumulée sur la production de bases de données, la bibliothèque avait conscience du coût que représentait la création des métadonnées. Les premiers projets de numérisation induisaient la production concomitante des métadonnées, créées ex-nihilo par les bibliothécaires, et des documents numériques. Dans certains cas, la production des documents numériques précédait celle des métadonnées avec un décalage parfois important entre les deux phases. Dans le cadre d'un projet de numérisation de masse, il était inenvisageable de reproduire ce type de circuit. Il s'agissait donc de disposer en amont de la numérisation de métadonnées (sous la forme de notices bibliographiques) que l'on pouvait alors convertir, " recycler ». Cette réexploitation des métadonnées induit alors une série de conversions. De façon schématique, cela implique :

- une analyse sur la qualité des métadonnées qui vont être réexploitées ;

- l'évaluation des corrections indispensables qu'il faut ou non apporter aux métadonnées pour qu'elles deviennent intéressantes et exploitables (en faisant alors la différence entre les corrections automatisables et celles qui ne pourront pas l'être), l'adaptation des modèles de conversion existants (de l'Unimarc vers le Dublin Core par exemple) ;

- la conversion effective des métadonnées.

Ces opérations induisent certes des connaissances des définitions de type de document (DTD) et schémas mais également une certaine maîtrise des 
outils d'analyse critique permettant d'évaluer la qualité des métadonnées ${ }^{4}$ avant leur conversion. Ces analyses permettent par exemple de savoir si certains pans de collections présentent des problèmes particuliers (les recueils factices par exemple), ou si les problèmes touchent certains catalogues rétroconvertis plutôt que d'autres.

Cette analyse effectuée, vient ensuite le temps de l'arbitrage : parmi ces erreurs, lesquelles doivent impérativement être corrigées ? Est-ce que ces corrections sont automatisables ? Dans le cas contraire, qui effectue ces corrections ? À quel moment?

Se pose ensuite la question de la conversion proprement dite. Lorsque l'on parle de réutilisation de métadonnées, il faut aussi entendre la possibilité de les convertir et de les encapsuler dans des schémas et DTD divers. Dans le cas de Numelyo, si l'on reprend l'exemple des documents numérisés via Google les notices en Unimarc sont converties en MARC21 puis en MARCXML pour être transmises à Google. Elles sont également converties en MODS pour être intégrées dans Numelyo et encapsulées dans des "notices » METS, elles sont également converties en Dublin Core non qualifié en attendant d'être intégrées dans un réservoir OAIPMH. Le pragmatisme doit être de rigueur puisqu'il ne s'agit pas de construire des modèles parfaits mais bel et bien des outils adaptés à des besoins et des objectifs.

\section{NUMELYO, UN WORK IN PROGRESS}

La construction de Numelyo ne saurait être racontée uniquement sous l'angle des métadonnées. Le travail effectué sur l'intégration et la mise en valeur d'autres contenus, la mise en place de services font aussi partie de la construction du projet. Numelyo se conçoit, à ce titre, comme un laboratoire et un work in progress.

Parmi les points que la bibliothèque souhaite développer, il faut noter l'importance que revêtent l'augmentation et la diversification des documents

4. Dans le cas de la numérisation des imprimés via Google, près de $80 \%$ des notices bibliographiques qui décrivaient le fonds provenaient de campagnes de rétroconversion. Le risque de rencontrer des erreurs gênantes voire bloquantes était plus important sur ces notices : risque d'erreur de lecture de la fiche bibliographique, risque d'erreur de saisie de la notice Unimarc. 
numériques au sein de Numelyo. Si la diversification des collections a été prévue dès la conception de Numelyo, la forte augmentation de la masse critique de documents mis à disposition pose toutefois des problèmes de stockage que la bibliothèque doit gérer.

Conçu dans le contexte du Web 2.0, le projet Numelyo aborde la question des publics et de leur participation de la même façon qu'il accorde une importance au développement des contenus et des contributions. Comme le précisent Nicolas Gros et Pierre Guinard ${ }^{5}$, l'extension du nombre de contributeurs constitue donc un point essentiel pour le développement de Numelyo. Cette question montre que le projet entre dans une nouvelle phase d'évolution. Alors que les aspects techniques tenaient une place fondamentale puisqu'il s'agissait de construire un outil, intégrer des documents et des métadonnées, développer des services et gérer des espaces de stockage, c'est aujourd'hui la question des contenus éditorialisés et des contributions qui est au centre du projet avec peut-être à la clé un passage de relais des techniciens vers les spécialistes des contenus... L'aventure Numelyo continue donc : à suivre !

LIBRE ACCÈS $\rightarrow$ BONUS NUMÉRIQUE

Galerie d'images extraites de Numelyo. http://www.enssib.fr/presses/catalogue/produire-des-contenus-documentaires-en-ligne-30

5. Nicolas Gros, Pierre Guinard, "Numelyo, la bibliothèque numérique de Lyon », Bulletin des bibliothèques de France, 2013, n 5, pp. 12-15. [En ligne] : <http://bbf.enssib.fr/consulter/bbf-201305-0012-002>. 


\section{4}

BALISES, LE WEBMAGAZINE COLLABORATIF DE LA

Nathalie Nosny

\section{BIBLIOTHĖQUE PUBLIQUE}

D'INFORMATION

Le projet de création du webmagazine de la Bibliothèque publique d'information (Bpi) faisait partie intégrante du projet d'établissement proposé dès 2011 par son directeur, Patrick Bazin. Il s'inscrit dans une approche volontariste de redéfinition de la bibliothèque comme un média, considérant que les compétences des bibliothécaires leur confèrent un rôle majeur dans notre société de l'information.

Ce projet s'est accompagné d'un remaniement complet de l'organigramme de la bibliothèque. Plusieurs départements ont alors été créés, dont le département « Lire le monde » qui a pour objectif d'aider les lecteurs à déchiffrer l'actualité et s'orienter dans un contexte de surinformation. Au sein de "Lire le monde », la mission principale du service webmagazine, en charge de la refonte du site Web de la Bpi et de la création du webmagazine, est d'organiser, coordonner et diffuser la production de contenus numériques sur le Web.

\section{UN PROJET TRANSVERSAL DANS SA CONCEPTION}

Dans le cadre du projet de création du webmagazine (et de refonte du site Web de la bibliothèque), un groupe de travail interne a été constitué en privilégiant des compétences complémentaires : maîtrise des outils et des usages du Web, connaissance des catalogues et des normes de structuration des données, compétences en termes de médiation numérique, compétences éditoriales et rédactionnelles, compétences en conduite de projets multimédias. Il a fonctionné en mode projet dès sa mise en place en avril 2012 à partir des grandes orientations définies par la lettre de cadrage de la direction, tout en favorisant la transversalité, notamment par le recours à des « experts » thématiques. 
D'un point de vue technique, la gestion de contenus devait s'appuyer sur une très forte structuration des contenus. Cela nécessitait d'intégrer les bases d'une sémantisation permettant une interopérabilité avec les contenus du catalogue de la Bpi et les contenus d'autres partenaires ou de bases externes. Les outils choisis devaient également privilégier l'usage et la mise en valeur des dernières technologies du Web (HTML5, CSS3, liens avec les réseaux sociaux, interactivité, etc.).

D'un point de vue documentaire, le webmagazine devait proposer des contenus éditorialisés, à l'attention du grand public, dans une visée pédagogique. Enfin, le projet devait être participatif et s'appuyer sur les contributions de l'ensemble du personnel de la bibliothèque, avec une perspective d'ouverture à des collaborations extérieures (partenaires, publics) à moyen terme.

Les objectifs déterminés par le groupe webmagazine pour mener à bien son projet étaient au nombre de sept :

- définir des publics-cibles ;

- définir une ligne éditoriale du webmagazine ;

- définir les besoins techniques de gestion et de diffusion des contenus ;

- définir un cadre graphique ;

- communiquer en interne autour du projet ;

- produire les contenus prévus dans la charte éditoriale du webmagazine pour le nourrir : contenus éditorialisés pour le magazine (articles, bibliographies, dossiers, etc.), contenus multimédias, intégration de contenus internes (base de connaissances de BiblioSésame, service de réponses à distance, blog du Cinéma du Réel, etc.) ;

- définir une organisation interne d'animation et de production de contenus en cohérence avec le portail documentaire. 
C'est principalement la réalisation de ces deux derniers objectifs qui nous intéresse ici, et je tenterai de détailler notre méthode, les obstacles auxquels nous nous sommes heurtés et les solutions que nous avons proposées.

\section{MANAGER LES CONTENUS}

La production de contenus, son organisation et sa coordination nécessitent, dans un mode collaboratif, une certaine formalisation sous peine d'avoir une production trop hétérogène. C'est dans cette optique que nous avons réfléchi, au sein du webmagazine et en collaboration avec nos collègues contributeurs, à des formats rédactionnels et à des outils de référence.

\section{UNE LIGNE ÉDITORIALE ET UNE CHARTE DES RÉDACTEURS}

Il a tout d'abord fallu préciser la ligne éditoriale du futur webmagazine : public visé, objectifs, thèmes abordés. Cette réflexion, également collective, a été menée avec les directeurs des différents départements de collection et validée en comité de pilotage.

En partant de cette ligne éditoriale, nous avons constitué un nouveau groupe de réflexion chargé de rédiger la charte des rédacteurs destinée à la fois à aider les contributeurs à mieux comprendre le cadre de leur contribution, mais aussi à définir les principes d'organisation des contenus (titres, chapô*, références, iconographie) et les principes de formalisation rédactionnelle (ton, vocabulaire, style).

À partir d'une trame réalisée par mon service, plusieurs réunions ont permis une discussion sur la définition de ces formats et leur adaptation à la réalité du quotidien de nos collègues en termes de disponibilité pour la rédaction, mais aussi de types de sujets envisagés. Par exemple, les « Portraits », qui nous semblaient un format simple, étaient plus éloignés des pratiques des acquéreurs alors qu'ils correspondaient bien aux attentes des programmateurs de manifestations culturelles dans la bibliothèque. 
L'exercice n'était cependant pas aisé car il s'agissait en grande partie d'anticiper des contenus dans un contexte qui n'était pas encore matérialisé : le futur webmagazine n'étant encore qu'à l'état de projet.

Nous avons cependant pu tester certains de ces formats et des outils au sein de la rubrique Les Dossiers de l'ancien site de la Bpi, malgré les contraintes inhérentes à notre CMS et à l'organisation du site tel qu'il avait été conçu. Cette rubrique a en effet accueilli des dossiers documentaires (dossiers complexes à plusieurs niveaux d'arborescence), des " mini-dossiers » (constitués d'une seule page), des portraits, des brèves. Cette période d'expérimentation nous a permis de les faire évoluer en fonction des difficultés rencontrées par les contributeurs (organisation de l'information, choix d'un angle spécifique, tendance à l'exhaustivité, ergonomie) et des premiers retours statistiques de consultation.

\section{DES OUTILS D'AIDE À LA PRODUCTION DE CONTENUS}

Alimenter un site nécessite une organisation et une planification au niveau de la création des contenus comme de leur exposition en ligne. Les articles publiés par la Bpi accompagnent l'actualité et se doivent donc d'être réactifs, même s'il ne s'agit pas d'une actualité à chaud mais plus d'une re-contextualisation de cette actualité. Il faut éviter de traiter des informations redondantes ou complémentaires et répartir la publication des contributions sur toute l'année en tenant compte de périodes plus tendues pour publier de nouveaux contenus comme les vacances d'été.

\section{Planifier}

Nous avons donc essayé de mettre en place des outils d'aide à la planification de ces contenus, dont un planning de production mensuel avec le nom des contributeurs concernés, les interlocuteurs dans le service webmagazine et le format d'article choisi. Ce document accueille également des informations sur les actualités culturelles de la Bpi et du Centre Pompidou ainsi que les grands moments de l'année afin de fixer des repères. Si ce planning est utile à l'équipe du webmagazine pour l'organisation du 
travail, il est encore peu utilisé par les contributeurs qui préfèrent passer par la conférence de rédaction pour l'enrichir (voir infra).

\section{Mutualiser la veille}

Chaque département pratique une veille sur l'actualité, mais à ses propres fins : valorisation, programmation, etc. Nous essayons de mettre en commun ce travail de veille à la fois pour rationaliser les efforts, mais aussi pour susciter une réflexion collective sur un sujet qui s'avère souvent plus riche qu'une réflexion individuelle, créant des passerelles parfois inattendues entre eux.

\section{créer un blog d'assistance}

En plus de ce planning et de cette veille sur l'actualité, nous avons créé un blog d'assistance technique et éditoriale qui rassemble des articles pratiques sur tous les aspects de la contribution. Cela permet aux contributeurs de retrouver les références techniques (quelle taille d'image pour une actualité ?) ou éditoriales (quels formats possibles pour une contribution ?) en plus des informations plus conjoncturelles sur la vie du site (réunions, correspondants, formations, documentation).

\section{choisir un outil de publication}

Enfin, il est essentiel de proposer aux contributeurs, surtout lorsqu'ils sont nombreux, un outil de publication (CMS) simple, ergonomique, attrayant. L'outil que nous utilisions était un CMS d'ancienne génération et ne possédait pas de nombreuses fonctionnalités auxquelles nous sommes aujourd'hui habitués sur le Web. Pour ses qualités de simplicité d'accès (on peut par exemple contribuer en front office*) et pour ses qualités de structuration des contenus, nous avons choisi Jahia.

Ces aides à la contribution se sont révélées précieuses au fil du projet même si leur mise en place implique au départ que l'on y consacre un certain temps. Ce sont des outils qui doivent constamment évoluer pour suivre les contributeurs dans leurs attentes et les évolutions du Web en 
général. Ils participent pleinement de la démarche d'accompagnement du contributeur qui est le cœur d'un projet collaboratif comme celui-ci.

\section{MANAGER LES PERSONNES}

Si l'organisation de la production de contenus est essentielle à ce type de projet, l'accompagnement des contributeurs l'est tout autant. Même si ces fonctions d'écriture, d'éditorialisation d'une actualité et de ressources documentaires sont désormais inscrites dans les fiches de poste, elles demeurent nouvelles pour beaucoup. Le bibliothécaire s'expose plus en rédigeant qu'il ne le fait habituellement. Ce type de publication en ligne est également spécifique : il s'agit d'une rédaction qui vise l'efficacité avant tout et qui s'éloigne d'une rédaction plus littéraire ou universitaire qui fait souvent partie de nos formations initiales. Quant aux outils du Web, qu'il s'agisse du CMS ou d'outils de visualisation de l'information, ils peuvent rebuter ou surprendre certains collègues qui ne les maîtrisent pas. Enfin, même pour les contributeurs les plus enthousiastes, il n'est pas toujours simple de s'approprier le cadre proposé, notamment en termes de ligne éditoriale, de mise en page ou de respect du droit d'auteur.

\section{DES MOYENS NÉCESSAIRES AU DÉVELOPPEMENT DU PROJET}

Indispensables, des moyens conséquents ont été accordés au projet. Le service webmagazine a été entièrement créé pour coordonner la production de contenus sur le Web. Il comporte 13 personnes dont 4 se consacrent entièrement à l'animation du site. L'ensemble des projets qu'il mène (animation éditoriale du site, médiation numérique, réponses à distance, webTv/webRadio, magazine imprimé, éditions, banque d'image) doit être piloté en synergie pour stimuler et valoriser la production de contenus de la bibliothèque.

Par ailleurs, l'équipe du webmagazine et l'ensemble des contributeurs ont bénéficié durant cette première année de nombreuses formations, certaines parfois très spécialisées comme la réalisation de vidéos ou des 
ateliers autour de la sémantisation qui nécessitent de mobiliser de nouvelles compétences.

En liaison avec le département informatique, le service a pu bénéficier d'outils plus performants : des ordinateurs plus puissants, des logiciels professionnels comme la gestion d'images fixes ou animées ; et du matériel spécifique a été acquis : caméra légère, enregistreurs audio-numériques. Enfin, la refonte du site et le choix du CMS ont nécessité un budget de 220000 euros auquel s'ajoutent chaque année le renouvellement de la licence de Jahia et une tierce maintenance évolutive pour le développement des fonctionnalités.

\section{UNE ORGANISATION INTÉGRÉE À L'ORGANISATION DE L'ÉTABLISSEMENT}

Pour soutenir le travail collaboratif des nouvelles équipes au sein des départements, inciter au partage des informations, susciter l'adhésion, coordonner les projets de publication, il a été nécessaire, outre l'appui de la direction, de mettre en place des instances régulières et des procédures adaptées.

\section{Les instances : conférence de rédaction et réunion de rédaction}

Une conférence de rédaction a pris la suite du comité Web existant. Elle rassemble un représentant de chaque service et/ou département. Au total 34 personnes se réunissent chaque mois. La conférence de rédaction a pour objectifs de faire circuler l'information, planifier les contenus, solliciter des contenus, prendre des décisions éditoriales, permettre des échanges techniques.

Le nombre élevé des participants en fait plus un outil d'information et de validation que de travail. Cependant, il y a peu d'absentéisme car une instance aussi transversale est une mine d'informations que les participants apprécient. 
Pour un fonctionnement plus réactif, nous organisons une réunion de rédaction plus limitée toutes les semaines, au sein du service. Nous y invitons les collègues susceptibles d'être concernés par les sujets du jour : rédactrice en chef du magazine papier, iconographe, collègue en charge de la post-production audiovisuelle, etc.

\section{LES PROCÉDURES (OU WORKFLOW)}

L'alimentation du site avait été jusqu'à présent basée sur le volontariat et sur une validation par des responsables de rubriques, de l'équipe webmagazine et du webmestre. Pour pouvoir produire des contenus de façon plus régulière et couvrir tous les domaines de la connaissance, nous avons dû revoir le workflow.

En nous appuyant sur l'organigramme existant, nous avons finalement opté pour une validation thématique des contenus par les chefs des services des collections concernés, pour plusieurs raisons : obtenir une plus grande reconnaissance du travail effectué par les contributeurs par leurs responsables, réguler la charge de travail entre travail interne sur les collections et contributions au site Web, impliquer les chefs de service dans le projet du webmagazine, donner plus de légitimité à la validation. Pour mettre en place ce workflow transversal, nous avons formé l'ensemble des chefs de service des collections. Même si nous devons encore faire un bilan de cette procédure sur les problèmes rencontrés, les lacunes éventuelles du système, et préciser encore le rôle de chacun, cette validation dans les services fonctionne globalement bien. L'équipe du webmagazine reste associée aux différents contenus, et poursuit l'accompagnement des contributeurs, tandis que les chefs de service peuvent intervenir pour arbitrer certains choix, prioriser les contributions et impulser des sujets en rapport avec la politique documentaire de l'établissement.

\section{un accompagnement et une formation au quotidien}

Notre service a mis en place dès le début du projet des sessions de formations courtes pour les contributeurs : formations à la contribution pour les débutants, traitement des images, intégration de contenus multimédias... 
Une fois la plupart des contributeurs formés, nous avons fait évoluer ces sessions en " ateliers pratiques » où les contributeurs sont invités à produire leurs contenus avec l'assistance de nos équipes. Cela leur permet d'avoir un temps dédié à cette activité qui est souvent délaissée au profit de la mission principale des contributeurs, d'échanger avec leurs collègues sur leurs pratiques et leurs approches de la contribution, d'être aidés et conseillés par l'équipe du webmagazine dans la construction et la réalisation de leurs contributions. Ces ateliers ont lieu chaque mois, un vendredi matin, avec des rappels réguliers du calendrier et si nécessaire des formations plus personnalisées en fonction des inscrits.

Ce système d'ateliers est apprécié et nous le poursuivrons sans aucun doute. Mais il n'empêche pas un accompagnement quotidien des collègues. Nous avons mis en place une boîte mail collective afin de pouvoir traiter efficacement leurs demandes et nous les rencontrons régulièrement sur leurs postes de travail pour déterminer avec eux les grands principes de leur contribution. Ce travail d'accompagnement, est le plus chronophage : il occupe au minimum $50 \%$ du temps de travail de l'équipe dédiée au site. Mais c'est un investissement essentiel pour garantir une adhésion des contributeurs, une bonne réactivité et des pratiques harmonisées.

\section{Imbrications : le quotidien, le court terme, le moyen terme...}

Un projet comme celui-ci, transverse et sous-tendu par un travail collaboratif, est un projet à long terme : il implique une mobilisation constante des équipes, des actions de formation continue aux nouveaux outils de publication numérique, une adhésion des collègues sans cesse renouvelée. C'est à la fois un projet visible pour le public à travers un site, mais aussi un projet plus souterrain d'évolution des métiers et des objectifs. Nous n'en sommes qu'à la première étape, l'étape visible, même si le travail d'accompagnement est déjà largement entamé.

La refonte du site Web de la Bpi, sur la base du travail réalisé une année durant par le groupe projet, a donc abouti à la réalisation de trois sites distincts faisant partie d'un même écosystème : le site institutionnel, point d'entrée du catalogue et de l'agenda de la bibliothèque ; le site 
professionnel, ouvert en partie à la collaboration avec les bibliothèques partenaires de la Bpi ; le webmagazine Balises.

Ces trois sites sont actuellement en cours de développement après l'attribution du marché à un prestataire extérieur, la société Sigma, qui les réalise sous Jahia.

Les contenus du webmagazine reprendront en grande partie les contenus déjà réalisés pour la rubrique Les Dossiers ${ }^{1}$ du site actuel, et seront complétés par des contenus nouveaux produits au cours du premier semestre et utilisant les différents formats définis dans la charte des rédacteurs. L'ouverture des sites et du webmagazine en particulier, prévue à la rentrée 2014, sera l'occasion de vérifier que ces contenus répondent à une demande du public et peuvent trouver leur place dans l'espace très convoité de l'Internet.

Une des limites de ce projet réside sans doute dans la masse critique indispensable de contenus nécessaires à la visibilité de ce magazine en ligne. C'est pourquoi il est prévu que la contribution s'ouvrira au-delà du personnel de la Bpi, à d'autres partenaires, bibliothèques, institutions culturelles, mais aussi au public en général (blogueurs particuliers ou grand public amateur). Cette nouvelle forme de contribution exige un cadre formel différent et pourtant complémentaire que nous allons devoir maintenant imaginer.

Un nouveau défi pour l'équipe du webmagazine qui continue d'animer avec enthousiasme cette production de contenus qui valorise à la fois les collections de nos établissements et les compétences de nos collègues.

1. < www.bpi.fr/fr/les_dossiers.html >. 


\section{PARTiE int}

\section{PRODUIRE EN \\ CO-CONSTRUCTION ET \\ EN RÉSEAU}

1. LES RÉSEAUX SOCIAUX : LE FONDS TRUTAT DE LA BIBLIOTHĖQUE MUNICIPALE DE TOULOUSE SUR FLICKR

par Jocelyne Deschaux et Patrick Hernebring

$++++++++++++++++++++++++++++++++++++++++++++++++++++++$

2. LES RÉSEAUX SOCIAUX : DIS-MOI QUI TU AIMES ET JE TE DIRAI QUI TU ES, ÉLABORER UNE POLITIQUE DE CONTENUS SUR FACEBOOK, L'EXEMPLE DE L'UNIVERSITÉ DE VERSAILLES SAINT-QUENTIN-ENYVELINES

par Magalie Le Gall

$++++++++++++++++++++++++++++++++++++++++++++++++++++++$

3. CONSTRUIRE UN DISPOSITIF PARTICIPATIF DE PRODUCTION DE CONTENUS EN MUSÉE

par Gonzague Gauthier et Sébastien Magro

4. FABRIQUER DES LIVRES NUMÉRIQUES : L'EXPÉRIMENTATION DU CENTRE DU LIVRE ET DE LA LECTURE EN POITOU-CHARENTES par Bruno Essard-Budaïl 


\section{1}

\section{LES RÉSEAUX SOCIAUX : LE FONDS TRUTAT DE LA BIBLIOTHĖQUE MUNICIPALE DE TOULOUSE SUR FLICKR}

par

Jocelyne

Deschaux

et Patrick

Hernebring

Entre avril 2008 et décembre 2013, la Bibliothèque municipale de Toulouse (BmT) a mis sur Flickr 4500 clichés issus du fonds de plaques photographiques d'Eugène Trutat (1840-1910), ancien directeur du Muséum d'histoire naturelle de Toulouse. Ils portent surtout sur les Pyrénées, mais ils abordent aussi des thématiques (sciences naturelles, anthropologie, archéologie, géologie, médecine...) et des lieux très variés. Cette richesse foisonnante de thèmes, associée à une grande valeur esthétique, semblait pouvoir séduire un public d'internautes.

Flickr, le réseau social de référence pour les amateurs de photographie, paraissait un lieu privilégié pour " arranger » un rendez-vous anachronique entre Trutat - l'expérimentateur du collodion, des émulsions instantanées, des ciels retouchés et des tirages à l'huile... mais aussi le pédagogue et l'auteur d'articles ou de monographies, - et des internautes, experts en retouche numérique d'images, fédérés sur le site par l'envie d'échanger autour d'une même passion, un peu plus d'un siècle plus tard. De plus, la dimension ethnographique des clichés semblait pouvoir faire naître des affinités entre habitants de jadis et d'aujourd'hui de mêmes terroirs.

\section{LA BIBLIOTHĖQUE DU CONGRÈS : LE PROJET THE COMMONS}

Le projet The Commons ${ }^{1}$ initié par la Bibliothèque du Congrès a abouti en janvier 2008 à la création d'un espace dédié du site où la Bibliothèque et les organismes publics pouvaient partager leurs collections iconographiques. Le but était de sensibiliser aux collections patrimoniales un public qui ne visiterait jamais la bibliothèque ni son site ; d'évaluer les éventuels bénéfices scientifiques et bibliothéconomiques de l'indexation sociale ou, 
plus généralement, des contributions des internautes, et d'acquérir une meilleure expérience de l'animation des communautés virtuelles.

Juridiquement, la mention "Aucune restriction de copyright connue » fut choisie pour répondre aux disparités des situations juridiques des différents pays, et pour pallier la difficulté d'établir sûrement l'absence de tout ayant droit.

Ce fut très vite un grand succès : 10 millions d'affichages en 10 mois ; une vingtaine de musées, bibliothèques ou centres d'archives y ont adhéré très vite. En avril 2008, première institution européenne à s'agréger au projet, et seule française jusqu'en septembre 2011, la BmT a mis en ligne un premier lot de 500 photographies d'Eugène Trutat. Aujourd'hui, 78 institutions y ont déposé leurs collections iconographiques.

\section{LA BIBLIOTHĖQUE DE TOULOUSE : LE PROJET FLICKR}

L'expérience Flickr ${ }^{2}$ s'inscrivait pour la BmT dans une dynamique alors naissante d'accroissement de la présence sur le Web et les sites sociaux. Ainsi, durant cette période, la BmT a ouvert trois blogs ${ }^{3}$, créé des profils sur de nombreux réseaux sociaux, proposé des gadgets d'interrogation de l'OPAC et recouru à l'emploi de logiciels libres. Dans le cours de cette exploration des enjeux liés au Web 2.0, Flickr, géant du Web social, présentait un terrain d'expérimentation très attractif.

De plus, la possibilité de géotaguer les images nous semblait particulièrement pertinente à appliquer à cette collection dont l'un des traits majeurs est de raconter la vie des territoires, d'en donner à voir les paysages et l'histoire.

\section{Des questions}

Si la mise en œuvre technique du projet était facile - de simples " copiercoller » de notices MARC existantes -, le contexte de démarrage du projet fut plus délicat : entrelacs complexe de perplexité, prévention et appétence, sentiment surtout de plonger dans l'inconnu.

2. < https://www.flickr.com/photos/bibliothequedetoulouse/>.

3. Blog à part : < blogapart.bibliotheque.toulouse.fr > ; le Blog de la Médiathèque Grand $\mathrm{M}:<$ grandm. bibliotheque.toulouse.fr/index.php? >; le Blog Le Mur de Berlin : < blog.lemurdeberlin.toulouse.fr >. 
Beaucoup de questions se posaient. Se " livrer » sans état d'âme à une entreprise commerciale telle que Yahoo ? Les clichés de Trutat, versés dans cet espace immense de partage, ne risquaient-ils pas d'être noyés dans un océan de photographies de vacances et personnelles diverses ? N'allions-nous pas en " perdre le contrôle » ? Quel serait l'accueil du public de Flickr ? Quelles seraient la valeur et la nature de ses contributions ? Saurions-nous provoquer l'envie de taguer ou d'annoter les photographies de notre galerie ? À quel point devions-nous, sans perdre notre identité, « devenir » Web 2.0 ?

\section{Fréquentation}

Les chiffres de fréquentation ont vite balayé une partie de ces doutes. Avec 3468917 d'affichages enregistrés au total (photographies, albums, et galerie confondus) depuis le début de l'expérience, une moyenne de 1500 clics par jour, des pics de fréquentation à 23000 vues sur une seule journée, des images particulièrement « populaires » affichées jusqu'à plus de 16000 fois, l'entreprise de diffusion culturelle, au moins sur le volet quantitatif, est un véritable succès.

En effet, notre galerie génère aujourd'hui encore un trafic de 4 à 5 fois plus important que celle d'un usager moyen. Un an après le lancement, notre compte se situait au cinquième rang en termes d'activité sur l'espace des Commons. Notre identité institutionnelle, statut atypique sur le site, et le caractère patrimonial très marqué des documents choisis sur notre galerie, ne semblent donc pas nous avoir pénalisés.

\section{Géolocalisation et albums}

Les modules d'exploration par lieu sur la carte du monde et de géotaguage, largement mis en avant dans le discours de communication de l'équipe du site, se sont révélés en pratique plutôt décevants : opérations de géotaguage parfois fastidieuses et lentes, sujets aux dysfonctionnements, recherche et exploration malcommodes, manque de finesse des statistiques de Flickr ne nous permettant pas d'apprécier la fréquence d'usage de cet outil.

Parmi d'autres fonctionnalités d'exploration, le site offre la possibilité de constituer des albums. Sous-ensembles documentaires, ceux-ci proposent un mode de navigation visuel, intuitif et convivial. Nous avons à 
ce jour 130 albums thématiques. Ils ont trouvé un véritable écho auprès des usagers et apparaissent comme un point de passage important dans le cours de la navigation sur notre galerie : ils représentent environ $10 \%$ du nombre de clics enregistrés sur le compte, soit un chiffre comparable à celui de l'accès direct aux images par les moteurs de recherche.

\section{LA PARTICIPATION DES INTERNAUTES?}

\section{Favoris}

Le premier niveau de participation de l'internaute - qui, le plus souvent, élude le volet social et n'utilise Flickr qu'en tant que banque d'images à voir ou à télécharger - consiste à créer des signets de favoris aux photos qu'il apprécie plus particulièrement. Ceci a pour effet de rassembler ces images sur une page spécifique de son compte.

C'est souvent à ce stade élémentaire de contribution que s'est limitée l'activité sur notre galerie. $62 \%$ de nos photographies ont été ajoutées au moins une fois aux " favoris".

Aussi, pour tenter de donner un visage à ce public mystérieux, nous avons analysé ces pages de favoris : les images nous ont semblé reliées par le fil directeur d'une démarche d'exploration de l'art et de la technique photographiques. La rencontre attendue a donc bien eu lieu : le vieux professeur a peut-être trouvé sur Flickr de nouveaux disciples...

\section{Groupes et expositions}

Une autre possibilité de construire des liens autour des images est de créer un groupe. Les groupes de Flickr permettent de collectionner des photos d'auteurs différents autour d'un thème et d'ouvrir un forum de discussion. Nous avons constitué le groupe «Eugène Trutat » dont nous sommes administrateurs. Par ailleurs, nous avons reçu une cinquantaine d'invitations, que nous avons très largement acceptées. Nous avons ainsi intégré le groupe des Commons, quelques groupes centrés sur la thématique des Pyrénées ${ }^{4}$; d'autres sur la photographie ${ }^{5}$, etc.

4. Les Pyrénées d'hier et d'aujourd'hui, Foto club pirineos (Photo Club des Pyrénées)...

5. . روصم تايجوي (Journal d'un photographe) ; I am a photovore ; Facing the Lens: Portraits of Photographers (Face à l'objectif : portraits de photographes). 
D’autre part, 291 de nos photographies ont été utilisées dans des expositions.

\section{Folksonomie}

L'une des interactions importantes entre internautes et éditeurs d'une galerie sur Flickr est cette possibilité de taguer les images. Ces motsclés, ou étiquettes, en s'agrégeant, ont constitué un système d'indexation collaborative réalisé par des non-spécialistes qui, dans la mesure où nous avons laissé la zone de tags vierge ${ }^{6}$, est le principal point d'appui de la recherche sur notre galerie.

La folksonomie est réputée se déployer sur un champ lexical plus centré sur l'utilisateur, avec éventuellement une dimension plus « affective » que la description " matière » proposée par les professionnels de la documentation. Au départ, le fait de créer par ce biais un nouveau maillage d'accès aux documents nous intéressait au premier chef. De plus, peut-être cela allait-il nous permettre de collecter des informations manquantes. En effet, un très grand nombre de lieux, édifices, sites naturels, personnages ou événements des clichés, tout comme la plupart des techniques, n'avaient pas pu être identifiés faute d'indication de la part de Trutat. En réalité, les attentes ont été très largement déçues : nous n'avons trouvé, dans les tags déposés, qu'une énorme majorité de termes descriptifs généraux (tels " arbre, route, maison »), plus partiels et de moindre qualité que l'indexation documentaire. En revanche, le nombre important de tags marque une activité certaine puisque $77 \%$ des clichés sont aujourd'hui tagués.

\section{BIBLIOTHĖQUE NUMÉRIQUE ET RÉSEAU SOCIAL : DES PUBLICS DIFFÉRENTS}

Rosalis ${ }^{7}$, la bibliothèque numérique mise en ligne par la BmT en octobre 2012, avec des moyens techniques, budgétaires et humains dépassant largement ceux investis dans le projet Flickr, irriguée par un référencement systématique des notices dans l'OPAC, dans Gallica, dans Isidore, ou

6. Nous n'ajoutons que le tag: «bibliothequedetoulouse » aux images. En revanche, nous copions dans la zone de description l'indexation des notices originales.

7. < http://rosalis.bibliotheque.toulouse.fr >. 
dans Google, avec la diversité et la richesse de ses contenus ${ }^{8}$, réalise des chiffres de fréquentation tout juste comparables à ceux de l'ensemble Trutat sur Flickr (1 750 par jour). Ils deviennent dix fois moindre en ne retenant que la partie " photographies anciennes » des collections numérisées. D'autre part, l'analyse des sources du trafic sur l'un et l'autre site indique sans ambiguïté qu'il s'agit de deux publics absolument distincts. Ainsi, malgré les très nombreux liens installés sur les pages des deux sites, il apparaît que moins de $1 \%$ des internautes passent de l'un à l'autre.

Les affluents principaux du site Rosalis relèvent pour une large part du " cercle des bibliothèques " ( $45 \%$ au total). De plus, l'examen des motsclés les plus fréquemment employés sur les moteurs de recherche pour aboutir au site démontre qu'ils gravitent pour l'essentiel autour de notre institution et de ses sites, et ne relèvent pas tellement d'une recherche d'informations plus générale qui les conduirait à croiser de façon fortuite les documents numériques de Rosalis. Les sites sociaux, quant à eux, ne génèrent que $2,41 \%$ du trafic sur le site.

Pour ce qui concerne le photostream de Flickr, $90 \%$ des clics proviennent de... Flickr lui-même. Ce sont donc des membres du site qui, s'appuyant sur les fonctionnalités et les gadgets sociaux mis en place, surfent et rebondissent d'image en image, et, surtout, de nouveauté en nouveauté.

En somme, nous trouvons du côté de Rosalis le public " naturel » des bibliothèques, et souvent de la $\mathrm{BmT}$, qui fréquente régulièrement les bibliothèques à la fois physiquement et virtuellement; et de l'autre, sans la moindre connexion entre les deux, un " nouveau public » apparu à la faveur de l'expérience et directement issu de l'immense communauté fédérée par Flickr.

Une mise en abîme troublante est que, au sein de ce dispositif relativement simple, le public de Flickr occupe la première place et représente environ $35 \%$ des consultations du patrimoine écrit - numérisé ou pas - de la BmT. Aucune action de promotion ou de diffusion de nos collections patrimoniales, sur les volets réels ou virtuels - expositions physiques ou virtuelles, pages de site, jeux en ligne, conférences, animations, communication en salle... - n'a su rassembler un public aussi large.

8. 60000 documents, 6 millions d'images environ. 
Les seules réserves, en définitive, concernent ce qui probablement suscitait au lancement du projet le plus d'attentes : la dimension sociale et ses apports. À cet égard, nous avons vu se reproduire sur notre galerie ce qui se passe sur l'ensemble du site. La pratique majoritaire sur Flickr est bien l'inactivité et seuls $3 \%$ des utilisateurs utilisent toutes les fonctionnalités de Flickr ${ }^{9}$. En revanche, nous avons bénéficié avec largesse de la " force des coopérations faibles » de Flickrr ${ }^{10}$, paradoxe d'une réputation finalement un peu usurpée de plateforme relationnelle, marquée de

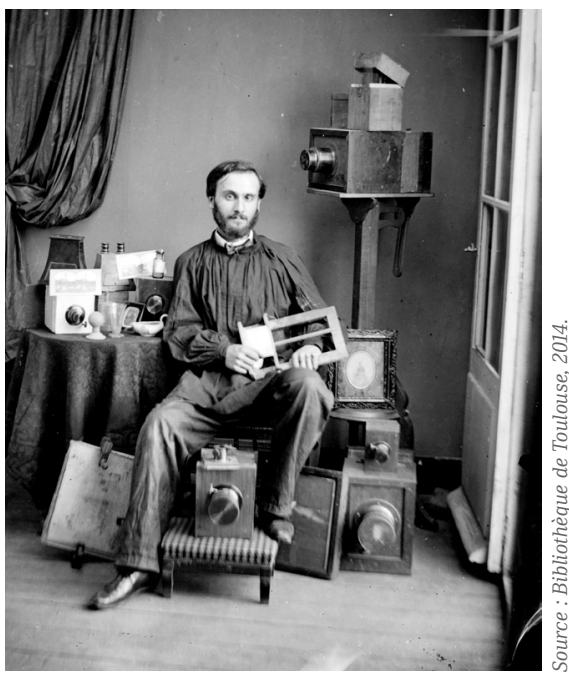

Ego, autoportrait d'Eugène Trutat dans l'atelier Vidal, vers $1860 .^{10}$ fait par une grande hétérogénéité dans le niveau de participation, mais agissant malgré tout comme un très puissant vecteur de valorisation.

LIBRE ACCÈs $\rightarrow$ BONUS NUMÉRIQUE

Galerie d'images sélectionnées dans le Fonds Trutat.

http://www.enssib.fr/presses/catalogue/produire-des-contenus-documentaires-en-ligne-30

9. Chiffres extraits d'un article de février 2008 du blog du Figaro : Suivez le Geek < http://blog.lefigaro.fr/hightechhigh-tech/ >.

10. Formule empruntée à Christophe Prieur, Dominique Cardon, Jean-Samuel Beuscart, Nicolas Pissard, The Stength of Weak cooperation: A Case Study on Flickr, 2008. [En ligne] : < www.academia.edu/5046262/The_Strengh_of_Weak_Cooperation_A_Case_Study_on_Flickr >.

11. < http://numerique.bibliotheque.toulouse.fr/ark:/74899/B315556101_TRUC1172 >. 


\section{2}

LES RÉSEAUX SOCIAUX: DIS-MOI QUI TU AIMES ET JE TE DIRAI QUI TU ES, ÉLABORER UNE POLITIQUE DE CONTENUS SUR FACEBOOK, L'EXEMPLE DE L'UNIVERSITÉ DE VERSAILLES SAINT-QUENTIN-EN-YVELINES

Avec plus d'un milliard d'utilisateurs, Facebook est l'un de ces outils incontournables qui pose la question de l'identité numérique d'un individu, d'une institution ou d'une marque. Les bibliothèques, en quête d'une image plus moderne et proche de leur public, l'ont bien compris. L'intérêt paraît encore plus évident pour une BU. Combien de fois ne voyons-nous pas l'interface bleue et blanche affichée sur un écran d'ordinateur lorsque nous déambulons dans les espaces ? Cependant, comment s'embarquer dans un tel projet quand on connaît les contraintes du monde administratif (étapes de validation successives, communication souvent rigide) et celles du management (formation et implication des équipes) ?

Lancées fin 2011, les pages des BU de l'université de Versailles SaintQuentin-en-Yvelines (UVSQ) ont permis de tester une nouvelle organisation en matière de communication sur la durée.

\section{UNE STRATÉGIE NUMÉRIQUE POUR L'UVSQ}

\section{DIFFUSER LA MARQUE}

Le décalage est souvent frappant : d'une part, les services de l'université s'adressent aux étudiants sur un ton très " administratif », d'autre part, ce fameux public étudiant de digital natives vit au rythme du langage SMS et de la communication instantanée. Il apparaît comme une urgence de nous défaire de la communication verticale top-down souvent invisible pour eux, pour changer l'image de la BU et de ses bibliothécaires. 
L'UVSQ est une université pluridisciplinaire de création récente (1991) qui compte environ 20000 étudiants pour 2140 personnels. Les 50 agents de la Direction des bibliothèques et de l'information scientifique et technique (DBIST) se répartissent sur six sites : Saint-Quentin-en-Yvelines, Versailles, Vélizy, Mantes-la-Jolie, Rambouillet et Boulogne. Suite aux réformes (LRU, création des PRES), l'UVSQ a développé une véritable stratégie digitale et gère sa " marque " pour répondre aux impératifs de visibilité et faire connaître l'offre de formation aux niveaux national et international. En termes de stratégie plus globale, ne pas prendre en compte un tel réseau constitue un risque de voir s'échapper l'e-réputation de l'établissement via notamment des pages non officielles. Il faut bien se dire qu'aujourd'hui, lorsqu'un étudiant choisit une université, il consulte les fils de discussion, les forums, les pages Facebook (FB), regarde les vidéos sur YouTube afin de se faire sa propre idée plutôt que de contacter directement l'université en question¹.

\section{LA MISE EN PLACE DU PROJET FACEBOOK}

\section{les objectifs de la DBIST}

La DBIST a formulé sa présence sur Facebook selon trois objectifs : écouter et aller à la rencontre des étudiants là où ils se trouvent, moderniser l'image de la BU et de ses agents, informer en temps réel sur un mode interpersonnel et non plus institutionnel. En résumé : créer du lien. L'idée est de systématiser l'articulation entre le programme d'animations des bibliothèques et son écho sur Facebook, dans une optique de valorisation systématique des collections et services afin de conduire davantage d'étudiants vers nos espaces, vers nos services et nos collections - présentiels ou en ligne -, vers la culture en général. Véritable banque d'accueil numérique, Facebook se présente comme un maillon intermédiaire entre la communication institutionnelle et le dialogue qui s'effectue avec l'usager en service public.

1. Caroline Chanlon, Community manager à l'université de Bourgogne : Universités : Facebook, la solution de facilité? : < http://www.huffingtonpost.fr/caroline-chanlon/universites-utilisationfacebook_b_2932538.html >. 


\section{calendrier et pilotage}

Le projet a donné lieu à trois validations (en mars, avril et juin 2011) en comité de direction avant son lancement. La possibilité offerte par Facebook de travailler sur une page ${ }^{2}$ sans la publier est précieuse car elle permet de tester des idées et de les soumettre à réflexion et correction. Peu avant leur lancement, nous les avons présentées à l'ensemble des collègues lors de deux journées du personnel en juillet et septembre 2011.

Deux agentes ont été chargées du projet : Dorothée Camus, ingénieur d'études à la BU de Saint-Quentin et moi-même, bibliothécaire à la BU de Versailles, toutes deux encadrées par la conservatrice responsable de la valorisation, Marie-Estelle Créhalet. La mission a été intégrée dans l'organigramme et dans les fiches de poste à hauteur de $10 \%$ des missions sous l'intitulé : Valorisation des collections et des services.

\section{Les formations du personnel}

La spécificité du projet repose sur l'intégration de l'ensemble des personnels de la DBIST. Il était hors de question que la communication sur Facebook reste l'apanage de quelques catégories A sélectionnées au préalable. La création d'un compte s'est faite sur la base du volontariat mais, projet de service oblige, la présence aux ateliers de formation était obligatoire pour tous. Sur quatre séances réalisées courant 2011, une quarantaine d'agents ont été formés et 31 comptes " Pro » créés. Les objectifs de ces ateliers étaient simples : faire le point sur les réticences, « dédramatiser » Facebook, former à la terminologie, à la manipulation de l'outil et aux paramètres de confidentialité, communiquer sur le projet pour terminer par la création d'un compte pour les volontaires. Facebook n'est pas du goût de tous et il faut affronter les réticences dès lors que vous obligez une équipe à suivre une formation. Pourtant, nombreux ont été ceux qui, hésitants au moment des ateliers, se sont depuis créés un compte Pro ${ }^{3}$.

2. Profil, groupe ou page ? En 2011, plusieurs bibliothèques étaient présentes via un « profil », beaucoup l'ont transformé en page. Les avantages concernent les statistiques de vos publications et la possibilité d'ajouter plusieurs administrateurs. En bref, le profil correspond à des personnes, la page à des entreprises, marques, institutions,... et le groupe s'apparente à un espace d'échange privé.

3. La création d'un compte pro ne signifie pas pour autant que vous disposez de rédacteurs réguliers. L'équipe publiante reste, somme toute, assez restreinte pour chaque page. 
Le passage à la Timeline ${ }^{4}$ a bouleversé l'organisation initiale. Jusque-là, les agents n'avaient pas besoin d'être administrateurs de la page pour publier. En mars 2012, les publications faites depuis les profils ne sont quasiment plus visibles sur les pages et, surtout, ne sont plus visibles sur le fil d'actualité des fans de la page. La solution envisagée : d'une part, publier en tant que page et, d'autre part, aimer et commenter depuis son profil « Pro ». Des formations plus approfondies ont été organisées rapidement et, au total, vingt agents sont passés administrateurs.

\section{ENCADRÉ 1 LE MÉMO DU PUBLIANT}

1. Je m’apprête à publier un nouveau message, est-ce que :

- Je suis bien sur la page de la BU et pas sur mon compte pro?

- Mon message est susceptible de faire venir à la bibliothèque ou d'inciter à utiliser une ressource de la BU ?

- Il s'agit d'une information à diffuser à nos publics ( $\neq$ information interne) ?

- Je (ou un collègue) suis (est) capable de répondre à une demande de précision?

- Je publie bien sur la page en tant que «BU de... »?

- Mon message n'est pas trop long ?

- Mon message est illustré d'une photo pour attirer l'attention? (si oui, voir point 2)

- J'ai bien supprimé l'adresse URL que j'ai copiée quand Facebook a affiché le lien propre?
- Mes collègues concernés sont déjà au courant de l'information que je publie?

2. Je m'apprête à publier une photo, estce que :

- Elle est libre de droits ? (Penser aux photos en Creative Commons sur Flickr...)

- J'ai tagué la photo avec l'auteur et la source?

- J'ai demandé l'accord des personnes figurant de face sur la photo si c'est moi qui l'ai prise?

3. Je m'apprête à faire un commentaire, ou aimer une publication, est-ce que :

- Je suis bien connecté avec mon compte pro et non pas en tant que «BU de...»?

4. La Timeline est une fonctionnalité de Facebook qui organise les contenus de chaque mur personnel sous forme de frise ante-chronologique. Pour les pages, cette fonction permet d'insérer du contenu, textes, images, vidéos, liens, sur un mode historique. 


\section{Les comptes « pro » au cœur du projet}

En 2010, suite au référentiel Marianne sur la qualité de l'accueil, nous avons décidé de jouer la carte de l'identification et d'adopter en service public des badges " chartés " comportant nos nom et prénom. Facebook devenant notre banque d'accueil numérique, il était logique de reproduire ce fonctionnement au travers de comptes Pro. L'idée était aussi que chaque agent puisse facilement communiquer par lui-même des informations sur le vif. Les critères exigés pour les profils sont restés simples : une photo de l'agent, ses prénom et nom suivi de "Pro » et l'UVSQ indiquée dans la rubrique employeur. Si ce type de fonctionnement est en contradiction avec les étapes successives de validation propres à la fonction publique, on assiste le plus souvent à une autocensure des agents, peu enclins à s'exposer publiquement, surtout vis-à-vis de leur hiérarchie.

\section{LE LANCEMENT DES PAGES DE LA BU EN 2011}

Le lancement des cinq pages ${ }^{5}$ s'est échelonné de septembre à novembre 2011. Les deux responsables du projet se sont déplacées sur les bibliothèques des pôles technologiques pour former les collègues n'ayant pu assister aux ateliers et lancer les pages.

Concernant la communication, un court article a été rédigé dans le magazine T'DACtu édité par la Direction de la vie étudiante de l'UVSQ et seules quelques affiches ont été placardées dans les BU. L'information a principalement été donnée aux étudiants par les personnels eux-mêmes lors de leur présence en service public.

5. Le choix a été fait de lancer une page par bibliothèque, excepté pour la bibliothèque médicale de Boulogne. Les usagers s'identifient par site et il nous paraissait essentiel de cibler l'information. Seule inquiétude : l'alimentation régulière des pages des « petits » sites tenus par un ou deux agents. 


\section{ENCADRÉ 2 EXEMPLES DE PUBLICATIONS SUR LES PAGES FB DE L'UVSQ}

Les services de la $B U$ :

- collections : buzz du mois, kits de l'été, nouveaux abonnements, sondage pour l'acquisition d'un manga ;

- formations : ateliers libres 12-14, visites de rentrée, partage de supports sur Slideshare ;

- autres services : navette, suggestions d'acquisitions, impressions à distance, prêt entre bibliothèques (PEB), rappel des consignes de sécurité dans la bibliothèque...

\section{Actions de valorisation:}

- événements : photos d'expositions, devinettes pour annoncer la projection d'un film... ;

- LibQual : RDV donné sous forme de devinette pour le lancement, liens vers l'enquête, photo de couverture faite avec un nuage de tags des commentaires des répondants, photo des boîtes des lots, un des items de l'enquête transformé en rébus, création d'un événement pour la « table-ronde café gourmand » organisée pour transmettre les résultats de l'enquête ;

- les étudiants donnent le « la » à la BU : post-it war, concours d'annonces micro.

\section{Communiquer au quotidien :}

- zoom sur l'actualité : mort de Stéphane Hessel, sélection d'ouvrages pour le Festival de Cannes, mort de Dennis Ritchie inventeur du langage $\mathrm{C}$... ;

- un petit mot en passant : vœux saisonniers, encouragements des examens... toutes les occasions sont bonnes!;
- les horaires : changements exceptionnels, fériés, horaires réduits... Pensez également à les modifier dans l'onglet « À propos »;

- les espaces de la BU : travaux, réaménagement, rappel du zonage des espaces ; - problèmes : chauffage, propreté...

\section{Saisir l'occasion :}

- valoriser le métier : photo d'une boîte de chocolats offerte par des étudiants, visites des espaces internes sur le mode " vis ma vie », photo des équipes lors du changement de SIGB, coulisses du récolement, photo d'une enveloppe envoyant un PEB à Santa Barbara... ;

- valoriser les étudiants : déguisements du carnaval, objets trouvés, associations étudiantes... ;

- célébrer les fans : des goodies ont été offerts aux $100^{\mathrm{e}}$ et $200^{\mathrm{e}}$ fans de la BU de Versailles, au $100^{\mathrm{e}}$ de celle de Vélizy et au $300^{\mathrm{e}}$ de Saint-Quentin.

\section{Le chantier du learning center} de Versailles :

- billets du blog tenu par l'équipe, compte Flickr de la BU ${ }^{1}$, informations en temps réel, inscription pour des visites VIP du chantier, photos des nouveaux mobiliers, des espaces et du déménagement, célébration de l'ouverture.

\footnotetext{
1. < http://www.bib-versailles.uvsq.fr/ > et $<$ http://www.flickr.com/photos/nouvelle_ bu_versailles/ >.
} 


\section{QUELS CONTENUS ?}

\section{La politique éditoriale}

Deux types de contenus ${ }^{6}$ sont à distinguer : l'alimentation et la réponse. Il faut toujours montrer que vous êtes là, derrière la page. Concernant l'alimentation, à raison d'une publication par semaine au minimum, il s'agit principalement à la DBIST de republier certaines actualités du portail documentaire, les billets du blog de chantier de la BU de Versailles, des informations plus ponctuelles très diverses ou du contenu multimédia. Les pages Facebook ne sont pas en concurrence avec le site Web des BU mais un point d'entrée supplémentaire avec, évidemment, l'espoir de toucher de nouveaux utilisateurs. Soit, deux questions à se poser systématiquement avant de publier :

- Est-ce que le message que je publie va faire venir l'internaute à la bibliothèque ou va-t-il l'inciter à lire nos ressources, papier ou en ligne?

- Si j’ai une demande de précision, serai-je en mesure d’y répondre?

\section{L'art de la réponse}

Qui dit visibilité dit aussi exposition... Les commentaires déposés sur les pages sont souvent positifs quand il ne s'agit pas de simples questions liées aux droits de prêt ou aux horaires. Les commentaires négatifs peuvent parfois nous être d'une grande utilité pour peser face à des politiques ou des fournisseurs. Nous avons ainsi directement transmis à l'entreprise en charge du ménage de l'université un message concernant la propreté des toilettes. Eux aussi sont exposés et doivent veiller à leur image.

6. Je renvoie à l'excellente Mind map d'Alain Marois : < http://fr.slideshare.net/amarois/quemettre-sur-une-fan-page-facebook-de-bibliothque $>$. 
EN CAS DE CRISE, QUE FAIRE ?

Toujours répondre. Dans le cas d'un dénigrement, il est important de rappeler les missions de service public qui sont les nôtres et dans le cas d'une critique fondée, d'apporter une réponse concrète. Et plutôt que de s'étendre - chacun défendant ses arguments à distance - il est préférable de proposer une rencontre. Dans la vraie vie, cette fois-ci.

\section{TO BE OR NOT TO BE "COMMUNITY MANAGER *" (CM)}

Le community management se définit comme l'animation et la gestion de communauté(s) en ligne ${ }^{7}$. Il s'agit de gérer la visibilité de son institution sur le Web, d'écouter ce qui se dit sur elle, de mettre en œuvre une stratégie de communication et de produire de l'information destinée à être partagée par les internautes.

\section{S'ORGANISER}

Les heures de connexion du grand public aux réseaux sociaux ne concordent pas forcément avec celles des horaires des bibliothécaires, puisqu'elles tournent autour des $19 \mathrm{~h}-22 \mathrm{~h}$ et le week-end. Certes, la fonction de programmation des publications peut faciliter la vie du CM, mais cela n'empêche pas de se connecter sur son temps libre (et ce point peut ne pas faire l'unanimité) pour vérifier qu'il n'y a pas de question urgente ou répondre à des commentaires offensifs. Le principe des réseaux sociaux étant l'immédiateté, rien n'est plus désagréable que l'absence de réaction.

\section{VEILleR ET ÉVALUER SA PAGE}

Facebook évolue très vite et un CM n'est jamais à l'abri des bugs. Évaluer une page suppose un temps que peu de CM ont à disposition. On se contente donc souvent des statistiques immédiatement accessibles : le nombre de fans et le nombre de personnes atteintes par une publication. Au lancement d'une page, ces chiffres virent parfois à l'obsession

7. < http://animerdescommunautesenbib.wordpress.com/ >. 
et peuvent paraître décevants. N'oubliez pas que vous vous lancez sur la durée et que des publications commentées ou tout simplement « likées » par vos fans sont aussi très largement appréciables. C'est l'engagement et l'interaction qui créent la valeur d'une communauté de fans et non son recrutement. Certains événements peuvent avoir des effets boosters inattendus : les post-it wars ${ }^{8}$ ont amené les étudiants à partager les pages pour faire voter leurs amis, les fermetures exceptionnelles liées à des problèmes de chauffage ont poussé des usagers à aimer la page pour savoir quand la BU rouvrirait (sic), l'ouverture de la nouvelle BU de Versailles a suscité la curiosité...

\section{S'INSPIRER DES ENTREPRISES POUR CRÉER DE L'ENGAGEMENT}

En mai 2012 est publiée sur la page de Nestlé une photo des desserts du restaurant d'entreprise de la marque. Avec près de 600 likes et 200 partages, il semble intéressant de suivre la façon de communiquer des grands groupes et de se demander ce qui marche vraiment auprès des utilisateurs. Par exemple, une photo va accentuer le stopping power* d'une publication et maximiser sa portée. Sur le fil d'actualité de l'usager, vous vous retrouvez en concurrence avec ses centres d'intérêt : musique, clubs de sport, grandes marques, séries TV... Pourquoi ne pas faire découvrir la bibliothèque sous un nouveau jour ? Avec des photos prises sur le vif par un smartphone, en mettant en scène une mascotte comme le squelette ${ }^{9}$ de la BU Santé de l'université de Reims, en se servant de Legos à la BU de Versailles. À vous de jouer !

8. Ou « batailles des post-it », est une technique artistique qui consiste à coller des post-it de tailles et de couleurs différentes sur des vitrages pour réaliser des fresques. Les guerres de post-it ont débuté en janvier 2011, à Montreuil, avec des employés des sociétés Ubisoft et BNP dont les immeubles se faisaient face. Leur bataille s'est achevée au mois d'août avant de s'étendre à d'autres entreprises et d'autres villes.

9. < https://fr-fr.facebook.com/busante.urca >. 


\section{ENCADRÉ 310 IDÉES À ADAPTER AUX BIBLIOTHÈQUES ${ }^{1}$}

- Posez des questions à vos fans pour les faire parler d'eux ;

- Posez des questions en suspens en utilisant les... ou

- Partagez des photos ou des vidéos prises sur le vif et favorisez ainsi la fonction de partage ;

- Prévoyez un calendrier de vos événements et adaptez les photos de couverture en fonction ;

1. < fr.slideshare.net/isabellemathieu/20ideescreerengagementfacebookjuin2012 >
- Rebondissez sur des témoignages d'usagers satisfaits, célébrez les bons résultats d'une enquête ;

- Organisez des concours avec un lot à gagner ;

- Publiez du contenu exclusif (coulisses) ;

- Donnez un RDV, publication récurrente (tous les [jours], à [heure] fixe) ;

- Annoncez une surprise à venir ;

- Utilisez des call-to-action en incitant à cliquer ou à commenter.

\section{3 ans après : quel bilan ?}

Trois ans après leur lancement, les cinq pages ne sont plus que quatre. En novembre 2013, pour cause d'inactivité, nous avons décidé de supprimer la page de la BU de Rambouillet pour la raccorder à celle de Vélizy. Depuis les vagues de formation de 2011 et 2012, tous les nouveaux personnels arrivant à la DBIST sont formés à Facebook et la plupart d'entre eux se créent des comptes professionnels. Trouver des idées d'animation des pages est essentiel, motiver les collègues sur la durée l'est encore plus.

Désormais, ce n'est plus seulement l'identité numérique du doctorant, du chercheur... mais bien celle du bibliothécaire qui s'inscrit dans l'identité numérique de son institution. En créant un profil professionnel, en complétant ses différentes expériences, en renseignant ses projets en cours ou en partageant sa veille, le professionnel des bibliothèques constitue un réseau de collègues, d'étudiants, d'enseignants-chercheurs... qui pourra déboucher sur des collaborations et des partages d'expériences nouvelles. 


\section{CONSTRUIRE UN DISPOSITIF PARTICIPATIF DE PRODUCTION DE CONTENUS EN MUSÉE}

\section{3}

par

Gonzague

Gauthier

et Sébastien

Magro

La présence des institutions culturelles et patrimoniales sur les réseaux sociaux numériques (RSN) a créé de nouvelles interactions entre l'institution et les visiteurs, entre les visiteurs eux-mêmes, mais aussi au sein de l'institution. Ces plates-formes permettent la mise en place de dispositifs participatifs, voire collaboratifs, qui associent les publics à la médiation, et nécessitent coopération entre les services et souplesse dans les étapes de validation, une transversalité au cœur d'une stratégie numérique pertinente et efficace.

Les dispositifs collaboratifs que construisent les institutions culturelles prennent place dans un univers prédéfini : il faut valoriser ses fonds documentaires, ses reproductions, ses créations d'auteurs ou d'artistes. Cela nous oblige à respecter des contraintes lors de la construction de nos dispositifs : du respect du droit d'auteur à l'interaction avec l'auteur lui-même, en passant par le respect de l'esprit du contenu lui-même. Par exemple, le Centre Pompidou a mis en place un dispositif participatif basé sur l'image, alors même que le système de droits de reproduction limite fortement l'usage de ce média. Sur Tumblr, la communauté est invitée à poster une photographie qui raconte une histoire autour d'une thématique à l'aide d'un personnage de papier, Paper toy ${ }^{1}$. Dernièrement, il s'agissait d'expliquer pourquoi la Crying Girl de Roy Lichtenstein pleure. Ici, l'œuvre est respectée et même enrichie, car le dispositif se base sur le détournement des narrations et de l'histoire de l'art à partir de matériaux photos. Il s'adresse à une communauté créative afin de compléter de manière inventive, et souvent pleine d'humour, le propos des visiteurs et amateurs.

En nous appuyant sur des exemples du musée du quai Branly et du Centre Pompidou, mais aussi d'autres institutions, nous nous efforcerons de

1. < http://centrepompidou.tumblr.com >. 
proposer des réponses claires, tirées d'expériences de terrain, à quelques questions entourant la mise en place d'un tel dispositif : comment créer les conditions de la participation ? Comment mobiliser sa communauté, en interne et à l'externe pour assurer une publication réussie ? Quels sont les outils de veille pour garder un œil sur sa communauté ? Quels sont les outils statistiques pour évaluer sa pratique?

\section{MOBILISER SON RÉSEAU : COMMENT IMPLIQUER TOUS LES ACTEURS DE LA CHAÎNE ?}

La première communauté $\mathrm{CM}$ est constituée par les agents de l'institution : avant de faire participer les visiteurs, il faut créer les conditions de la participation de l'ensemble des agents. La mise en place d'un réseau de référents internes permet de relayer les demandes issues des RSN et d'y apporter des réponses. Ce groupe peut également participer à la création de contenus originaux. Au Centre Pompidou, il s'est constitué autour d'outils qui leur permettent de réaliser plus simplement et rapidement des tâches dans leurs missions. En revanche, si le site Internet de l'établissement est alimenté par un comité éditorial, le plus simple est de s'appuyer sur ces contributeurs : ils sont formés à écrire pour le Web, sensibilisés aux impératifs techniques et connaissent la temporalité en ligne, souvent plus courte que celle de l'institution. Au musée du quai Branly, le community manager s'appuie sur les membres du comité éditorial, initialement désignés par leurs directions respectives. Ainsi, plusieurs agents participent sous la coordination du chargé de projets nouveaux médias. S’il administre la page Facebook du musée ${ }^{2}$, les deux autres pages permanentes sont gérées par les services pertinents : quatre agents de la Direction des publics pour la page des soirées Before et quatre agents du Département du patrimoine et des collections pour la page de la Médiathèque et du salon de lecture Jacques Kerchache ${ }^{3}$. En outre, les conservateurs, les commissaires des expositions et les agents

2. < https://www.facebook.com/museeduquaibranly >.

3. < https://www.facebook.com/mediathequeetsalondelectureduquaibranly >. 
de la régie sont de précieuses personnes ressources pour rassembler du contenu de qualité.

Un exemple : les lundis, le musée utilise le mot-dièse \#jourdefermeture sur Twitter pour faire entrer les visiteurs dans ses coulisses. De nombreuses autres institutions l'utilisent également pour partager photos, vidéos ou informations exclusives sur la vie au musée lorsqu'il n'est pas accessible aux visiteurs. Si, lors de l'apparition du dispositif, il était indispensable d'en justifier le contexte, les motivations et le fonctionnement, les agents du musée proposent aujourd'hui spontanément des contenus au CM. Une fois les conditions de la transversalité créées, les outils de la collaboration sont la prochaine étape pour procéder à la mise en place d'une politique cohérente sur les RSN.

\section{PUBLIER : QUELS OUTILS POUR COLLABORER ?}

Les premiers outils de publication sur les réseaux sociaux sont les platesformes elles-mêmes : vous pouvez poster directement sur Facebook, Twitter ou encore Pinterest. De plus, il est possible d'utiliser les RSN eux-mêmes comme outils collaboratifs pour assurer la cohérence du community management : à la BnF, les six animateurs des comptes Gallica utilisent un groupe Facebook privé pour échanger et préparer leurs publications. Certaines applications tierces permettent également de programmer vos publications et, sous certaines conditions, de collaborer, avec la mise en place de plusieurs comptes utilisateurs. Seesmic ou Tweetdeck, permettent cette fonctionnalité qui peut s'avérer très utile lorsque plusieurs agents interviennent sur un même compte Twitter : au Centre Pompidou et au musée du quai Branly, c'est Hootsuite qui est utilisé, car il permet de gérer plusieurs comptes de réseaux sociaux, de programmer, de réduire les liens et propose un tableau synthétique des publications prévues.

\section{Quel calendrier ?}

Établir un calendrier de publication est une étape importante pour gagner du temps, en n'oubliant pas de laisser de la place à l'imprévu propre au Web. Vous aurez une meilleure visibilité avec un planning, même approximatif, des thématiques que vous souhaitez mettre en avant et du moment où vous les publierez. Ce calendrier peut être utile dans le cas d'intervenants 
multiples ou de validations internes. Enfin, les publications programmées offrent aussi la possibilité de maintenir une activité en ligne pendant les week-ends et les congés. Depuis 2013, Facebook permet de planifier une publication directement sur le site, ce que ne permet pas Twitter, pour lequel il est nécessaire d'utiliser l'un des services tiers cités plus haut.

\section{COMMENT CRÉER DES STANDARDS POUR LA COLLABORATION ?}

\section{Établir des chartes éditoriales}

S'il n'existe pas de charte, ni pour le site Internet, ni pour les réseaux sociaux numériques, il est préférable d'en mettre une en place. Dans un premier temps, quelques grands principes suffisent, il n'est pas impératif de trop détailler car le document pourra être complété par la suite : qualité des visuels, normes de mise en page et ton. La plupart des plates-formes exigent au minimum une photo de profil et un visuel de type " bannière ", profitezen pour valoriser votre logo ainsi que des éléments phares de votre institution (architecture, œuvre, etc.). Assurez-vous d'utiliser une même norme pour les citations, noms propres ou noms des ouvrages que vous évoquez. En complément de cette identité graphique, il faut mettre en place une identité discursive. Vos choix sont nombreux et n'appartiennent qu'à vous : tutoiement ou vouvoiement, abréviations et jargon, niveau de langue soutenu ou plus proche de la langue parlée, etc. L'important ? Qu'ils soient cohérents dans le temps et avec "l'image de marque » de votre institution. Même seul, il n'est pas toujours évident de se souvenir des quelques règles que l'on s'est données : il faut régulièrement les relire pour se les remettre en mémoire. Si vous êtes plusieurs à intervenir, faites un recueil des messages postés les plus exemplaires et ajoutez en tête quelques conseils et impératifs, pas plus! De toute façon, c'est la pratique qui entérinera les formules.

\section{utiliser un « planning conversationnel »}

La charte éditoriale permet d'organiser la prise de parole thématique de l'institution sur les réseaux sociaux : qui dit quoi ? À quel moment ? Là encore, ce travail doit correspondre à vos contenus et à votre communauté. Au-delà de la grille de publication adaptée aux institutions de taille 
moyenne, les plus grandes structures peuvent désigner une personne pour centraliser le travail au cas par cas. Pour ce faire, au Centre Pompidou, nous utilisons un « planning conversationnel » qui permet de s'adresser à des segments de publics en fonction de leurs thématiques favorites.

\section{ENCADRÉ POUR UNE EXPOSITION (CENTRE POMPIDOU)}

- définir une période sur laquelle travailler : fixer une date en fonction des impératifs de communication, de l'agenda culturel parisien ou français et de la programmation du Centre Pompidou. L'importance de la conversation va conditionner le pourcentage de messages quotidiens mobilisés. Pour une nouvelle exposition : $50 \%$ des messages pendant 5 jours ;

- les messages du début font connaître l'exposition, puis l'expliquent dans les grandes lignes. Les messages de fin per- mettent de comprendre une œuvre, puis de la faire discuter par les internautes ;

- les messages sont qualifiés, puis des mots-clés sont attribués en fonction des sujets abordés. Ils permettent ensuite de cibler les contenus qui vont être publiés d'une part, et les "influenceurs »* qui seront recensés d'autre part. À ce moment, le CM fait valider la démarche par la personne en charge de la programmation (ici, le conservateur ou son équipe) ; il procède ensuite à la rédaction, puis à la programmation des messages.

\section{COMMENT ABORDER UN CONTEXTE MULTICULTUREL ?}

$\mathrm{Au}$ Centre Pompidou, le planning conversationnel permet également de s'assurer que toutes les communautés thématiques sont approchées régulièrement, tandis que le tableau des "influenceurs » recense les centres d'intérêt de chacun des contacts numériques via un mot-clé. Par exemple, un blogueur, visiteur fréquent d'expositions photo, se voit attribuer « photographie » et " exposition », tandis qu'une personne présente sur Twitter, avec un grand nombre de followers retwittant régulièrement des contenus " architecture », se verra attribuer ce même mot-clé. Le premier pourra être mobilisé lors de la production de contenus, tandis que l'autre sera mobilisé dans une volonté de diffuser fortement un message. Ces outils 
permettent également de conserver un historique de la relation de l'institution à ses publics. L'approche est éditorialisée, car nous ne considérons pas pouvoir parler à des communautés de manière uniforme : elles ont une culture et des références différentes qu'il faut respecter lors des échanges. Pour cette raison, si vous êtes en capacité de réaliser ce type d'approche, il peut être intéressant de créer des comptes distincts : vous ne publierez pas les mêmes contenus au même moment.

Si vous ne réalisez que des traductions de vos messages, l'approche multilingue au sein d'un même compte peut suffire, à condition de publier une majorité de messages dans la langue la plus parlée au sein de votre communauté. Les messages en langue étrangère vous donneront une image internationale qui ne vous nuira que rarement. Pour s'assurer d'une meilleure visibilité, il est également important de trouver le bon moment pour poster, notamment en tenant compte du décalage horaire. Par exemple, des publications en anglais dans la soirée vous permettront de toucher des publics internationaux, notamment en Amérique du Nord et dans le Pacifique. La page Facebook de Photoquai, biennale des images du monde organisée sur le quai Branly, a permis au musée de tester des publications bilingues, grâce à des intervenants formés, une expérience qui s'est avérée très porteuse sur le plan international.

\section{COMMENT INTERAGIR AVEC LES PUBLICS ?}

S'il n'est pas utile de réaliser un contrôle $a$ posteriori des contenus postés par les référents, cela peut même se révéler très improductif car démotivant, voire tout à fait impossible. Il est donc tout aussi efficace d'apprendre à réagir aux diverses situations qui peuvent se présenter à vous. Qu'une erreur ait été produite par le CM ou l'un de ses collègues, ou qu'un contenu de l'institution ait motivé l'ire de vos fans, les commentaires risquent de s'enchaîner. La première règle consiste donc à lire tous les commentaires. Évitez de les survoler, vous pourriez manquer une ironie assassine que vos autres fans relèveraient ensuite avec entrain. Lorsqu'elle ne révélera aucun danger imminent, cette lecture vous apprendra de nombreuses choses sur votre communauté : se parle-t-elle à elle-même ou seulement à vous ? Réagit-elle de façon plus positive que négative ? Qui sont les principaux contributeurs ? Autant d'indices que 
vous pourrez ensuite mettre à profit si vous devez répondre à un débat houleux.

Même s'il n'est pas possible de toujours anticiper quand vous postez un contenu, faites en sorte d'en avoir tout de même un second qui puisse préciser le propos d'une façon différente : l'interview d'un artiste fait débat quant au sens de son œuvre ? Postez un dossier pédagogique. Non seulement votre réponse aura du sens, mais elle vous permettra de ne jamais être pris à parti personnellement. De plus, les polémistes seront repérés par les autres membres de la communauté, face aux débatteurs sérieux. Une autre règle simple : plus le contenu est sensible, plus vous aurez tendance à le publier en journée. Cela peut être néfaste en termes d'audience, mais si une polémique explose sur les RSN, pensez-vous pouvoir avertir les experts du contenu incriminé à $19 \mathrm{~h}$ un vendredi soir ? En tout état de cause, il faut que votre community management soit élastique : un type de contenu et une approche éditoriale, plutôt que les messages eux-mêmes, doivent être validés, pour que chaque acteur de la chaîne puisse réagir en toute connaissance de cause si nécessaire. Si cela n'est pas compris en interne, vous pouvez expliquer que, par votre veille quotidienne, le CM est celui qui connaît le mieux sa communauté et peut réagir avec le plus de finesse.

\section{ENTRETENIR UNE VEILLE : QUELQUES CONSEILS}

La veille à travers les réseaux sociaux ne diffère pas beaucoup des pratiques de veille sur supports imprimés qui vous sont sans doute familières. Avec un peu d'organisation, Twitter est l'un des outils de veille les plus précis et les plus puissants. Pour bien démarrer, listez quelques thématiques que vous souhaitez surveiller. Il est préférable de commencer par un petit nombre, puis vous pourrez augmenter votre champ d'investigation. La plate-forme de micro-blogging permet de créer des listes pour classer et consulter vos abonnements, ce qui est très pratique pour rester concentré sur une thématique. Progressivement, identifiez plusieurs comptes à suivre autour de ces sujets, qu'il s'agisse d'utilisateurs individuels, de comptes d'institutions ou de marques, ou encore de sites 
d'information. Par exemple, au Centre Pompidou, nous paramétrons des fils de recherche Hootsuite, en fonction des mots-clés de l'actualité du Centre Pompidou et du monde culturel, renouvelés de manière périodique (au rythme de notre programmation, tous les 3 mois). Le suivi des blogs des influenceurs recensés dans le tableau nourrit également cette veille, ainsi que la lecture fréquente des sites connus du secteur traité.

\section{ÉVALUER SA PRATIQUE : LES OUTILS DE STATISTIQUES}

D'autres services Web de ce type facilitent également le travail du CM qui met en place des dispositifs collaboratifs. Par exemple, il est essentiel de pouvoir contrôler l'émission de ses messages, autant que les taux de réponse, de reposts, de mise en favoris. Les outils de publication tels que Hootsuite créent des rapports statistiques répondant à ce besoin. D'autres outils existent, dans des versions gratuites ou payantes : Crowdbooster ou TweetStats permettent de comparer des comptes et leurs résultats. Au-delà de ce contrôle de la production et de sa réception, vous aurez peut-être besoin de connaître l'audience et la notoriété de vos comptes. Pour ce faire, l'outil le plus connu est Klout, qui agrège les différents réseaux sociaux d'une même marque pour faire un calcul global : en ajoutant de nouveaux comptes RSN, en réalisant un travail d'élargissement des abonnés aux comptes, Klout va augmenter. Cet indicateur peut être rapporté à la direction pour montrer son audience. Mais toutes ces informations ne sont que des indicateurs assez peu signifiants. Prenez les choses dans le bon sens : identifiez vos objectifs. Cherchez ensuite l'outil qui vous permet d'évaluer la réussite de ceux-ci. Parfois, les outils ne correspondront pas tout à fait, prenez le meilleur et lisez ses résultats de manière contextuelle et critique. Par exemple, si vous tentez de fidéliser votre communauté, tournez-vous vers PostRank Analytics. Au Centre Pompidou, pour le projet \#CheckPaint décrit ci-dessous, l'objectif était surtout celui de la médiation : l'indicateur mis en place concernait le nombre de messages produisant du contenu en rapport aux documents proposés. Il fallait analyser ces messages, en estimant leur pertinence et les classer dans un tableur. Le résultat conditionnait, jour après jour, les rédactions des messages suivants. 


\section{COLLABORER ENTRE INSTITUTIONS}

L'évaluation peut aussi être réalisée pour de potentiels partenariats, à travers des services qui permettent de calculer l'audience de comptes Twitter, par exemple. Ainsi, il est possible de choisir des partenaires en fonction de leurs publics, de manière à profiter de l'engagement de ceux-ci sur une thématique. Dans le cadre de \#CheckPaint, une série sur les RSN qui mettait en scène des personnages fictifs à travers cinq comptes Twitter, le Centre Pompidou a réalisé des partenariats qui permettaient d'enrichir le profil des personnages. Un personnage, adepte des cultures urbaines et des phénomènes de mode a par exemple échangé avec le compte du Forum des Halles. Un autre, s'interrogeant sur l'histoire du musée, a reçu des réponses issues d'archives de Gallica. Ainsi, Gallica a montré l'usage de son site aux spectateurs de la série, qui étaient eux-mêmes régulièrement incités à échanger et commenter des contenus. À partir d'une histoire, les publics des deux institutions sont amenés à découvrir des contenus différents mais proches de leurs centres d'intérêt.

\section{VERS L'INCLUSION DES PUBLICS ?}

Les réseaux sociaux constituent pour les musées, comme pour les bibliothèques, une nouvelle façon de présenter les ressources, et surtout de faire interagir les publics avec celles-ci. L'inclusion des publics dans des dispositifs de production de savoir est l'un des points essentiels du développement numérique des institutions. Laisser la main aux visiteurs induit a minima une observation de leurs pratiques, et pourquoi pas l'adoption de certaines d'entre elles. À travers les dispositifs ainsi mis en place par l'institution, cette communauté d'usagers produit des contenus qu'un bon $\mathrm{CM}$ valorisera dans le but d'en motiver l'engagement.

Peut-on cependant imaginer un dispositif plus poussé ? Les institutions reconnaissent de plus en plus comme un enrichissement la polyphonie que permettent ces contenus issus de plusieurs cultures différentes (scientifiques, amateurs, etc.). Mais celle-ci reste contrainte à un cadre produit par l'institution elle-même. La prochaine étape de l'inclusion des publics dans les contenus culturels ne serait-elle pas non plus seulement leur co-production, mais bien une co-construction des dispositifs participatifs eux-mêmes ? 


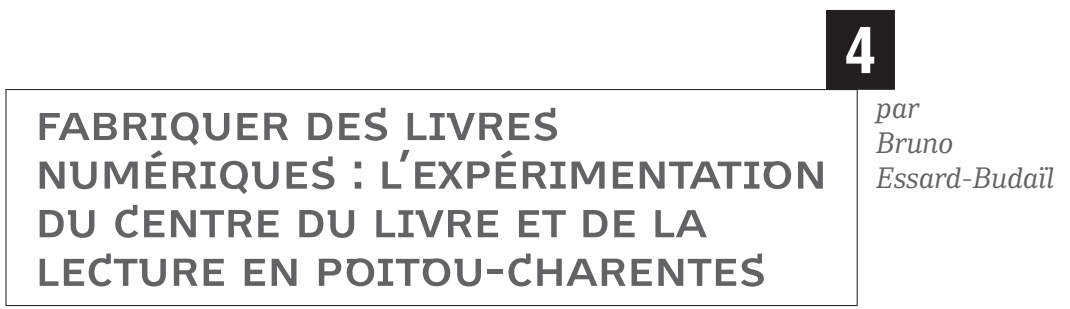

Les orientations du Centre du livre et de la lecture (CLL) définissent le cadre des expérimentations liées à la fabrication de livres numériques. L'une d'elles précise : " Faire des technologies de l'information et de la communication, du numérique et de la robotique des outils au service du développement de la lecture, et des acteurs de l'écosystème du livre »; et une autre : " La lecture facteur de développement territorial et humain et de l'écosystème du livre à l'ère numérique ».

Le CLL se définit en partie comme un laboratoire et un espace d'expérimentation, de pédagogie et de formation en région pour cet écosystème du livre et de la lecture, et tous les acteurs qui le constituent.

Concrètement, cela se traduit par un cycle de rencontres et formations : " Lire Écrire Penser et Conserver dans un monde numérique » (LEPC). Sa cinquième édition est programmée pour le printemps 2015 ; des outils en ligne ${ }^{1}$ (site du CLL, Scoop.it/Djébalé - lectures numériques - et Scoop.it/L'enfant et les écrans), et des expérimentations, notamment dans une activité en pleine émergence : la création de contenus.

Deux questions se posent alors : pourquoi et comment les bibliothécaires de ce début de $\mathrm{XxI}^{\mathrm{e}}$ siècle doivent-ils créer des contenus?

Nous ne nous sommes pas attardés sur le « pourquoi » tant il semble pertinent de travailler sur la valorisation des collections (sur les collections patrimoniales mais pas seulement), et de mettre en place une politique de médiation culturelle, mais plutôt sur le " comment ». Quelles solutions techniques permettraient une mise en œuvre simple, peu coûteuse

1. Site du CLL Poitou-Charentes : < http://www.livre-poitoucharentes.org >. Scoop.it/Djébalé (lectures numériques) : < http://www.scoop.it/t/djebale >. Scoop.it/L'enfant et les écrans : < http:// www.scoop.it/t/l-enfant-et-les-ecrans >. 
et envisageable pour tout type d'établissement : de la BMVR à la petite bibliothèque rurale?

Il ne s'agit pas ici de présenter une solution universelle mais le résultat d'expérimentations qui prennent en compte deux paramètres :

- pour le livre numérique : les bibliothécaires n'ont pas besoin de prérequis sinon l'utilisation d'un traitement de texte et l'utilisation de logiciels libres ${ }^{2}$;

- pour le livre numérique enrichi : il était acté dès l'origine du projet que le CLL avait la maîtrise totale des contenus.

À travers deux exemples, le livre numérique et le livre numérique enrichi, nous allons cheminer à la découverte de ces objets de médiation particulièrement efficaces.

\section{LE LIVRE NUMÉRIQUE ${ }^{3}$ AU CLL : 2011-2013}

Le projet d'expérimentation de liseuses en bibliothèque s'appuie sur le constat de Michel Fauchié (président de l'Association pour le développement des documents numériques en bibliothèque, $\mathrm{ADDNB}$ ) : "C'est le rôle des bibliothèques d'accompagner les lecteurs dans la découverte de nouveaux supports ", mais pas seulement. L'intérêt que nous y voyons ne se focalise pas sur l'objet lui-même mais sur le nouveau vecteur de lecture. La question posée d'entrée est donc celle des contenus. L'expérimentation mise en place fin 2011 s'appuie bien évidemment sur d'autres expériences qui ont précédé dans d'autres départements, d'autres lieux. Le projet prend en compte notamment les critiques visant les contenus : la plupart des premières expérimentations proposaient des ouvrages choisis parmi les « classiques » de la littérature car ils sont libres de droits et gratuits.

2. Les logiciels que nous utilisons sont certes gratuitement téléchargeables mais nous proposons toujours que les collectivités s'engagent financièrement (même très modestement) lorsqu'elles les utilisent.

3. Question de terminologie : le terme « livre numérique » sera employé pour les ouvrages au format epub lisibles sur liseuses et celui de " livre numérique enrichi » pour les ouvrages lisibles sur iPad et tablettes. 
Nous chargeons alors dans les liseuses des titres d'auteurs du patrimoine régional (plutôt des titres méconnus), des auteurs liés à notre actualité et/ ou à celle des bibliothèques partenaires (festival, expositions, rencontres d'auteurs...), et enfin des nouveautés (plutôt sans DRM).

En lien avec cette expérimentation d'autres pistes sont explorées :

- dans les bibliothèques : mise en place de Bibliobox* qui permettent de proposer des contenus numériques gratuitement et anonymement (textes, musiques, films) y compris dans les équipements ne possédant pas de connexion ou de wifi ;

- hors les murs : par le biais d'affiches apposées dans les commerces, administrations, lieux de passages, utilisation des QR codes* afin de proposer les premiers chapitres d'ouvrages d'éditeurs de la région et d'inciter les lecteurs à venir emprunter le livre dans une bibliothèque comme c'est le cas pour le réseau " Cœur du Bocage » de l'agglomération de Bressuire dans les Deux-Sèvres ${ }^{4}$.

Proposer une offre numérique liée à une manifestation peut rapidement devenir un casse-tête car les documents souhaités n'existent pas toujours en numérique. C'est le cas pour un catalogue d'exposition, pour un manuscrit, pour un ouvrage non publié.

\section{MISE EN CEUVRE D'UN LIVRE NUMÉRIQUE PAR LES BIBLIOTHÉCAIRES}

À titre d'exemple, explorons le cas d'une manifestation littéraire : un réseau de bibliothèques souhaite organiser une exposition autour des œuvres de Maurice Renard et offrir aux usagers et aux visiteurs de l'exposition des livres numériques. Les ouvrages du maître du merveilleux scientifique sont, pour les plus connus, disponibles gratuitement sur de nombreux sites. Les bibliothécaires souhaitent cependant proposer un ouvrage inédit de l'auteur sans que cela soit financièrement douloureux.

4. < http://mediatheque.coeurdubocage.fr/coeurdubocage.fr/opac?id_profil=27 >. 
Une fois les textes rassemblés (dans cet exemple ce sont des nouvelles policières glanées dans les collections du quotidien Le Matin sur Gallica), les OCR nettoyés (le temps de lecture/correction est souvent important), on se retrouve au mieux avec un fichier Microsoft Word ou Libre office, au pire avec un PDF, mais pas encore avec un livre numérique.

La démarche de création d'un livre numérique en epub* proposée par le CLL se veut la plus simple possible : construire un fichier avec du texte et une image de couverture, sans inclusion d'objets (dessins, tableaux, films...) sans effet typographique et sans mise en page complexe, mais qui peut comporter des liens, un sommaire interactif et des notes.

Ce n'est pas la seule méthode existante mais elle a le mérite de demander peu de prérequis et de ne pas être coûteuse.

Lors d'une formation courte (deux jours en novembre 2013) nous avons proposé à une dizaine de stagiaires une matinée ${ }^{5}$ de présentation du livre numérique (histoire, diffusion distribution, aspects juridiques) suivie de trois demi-journées ${ }^{6}$ consacrées à la fabrication du livre numérique. Chaque stagiaire disposait d'un ordinateur sur lequel étaient disponibles les outils nécessaires à la création du livre numérique.

Après cette courte formation, les bibliothécaires sont à même de fabriquer les livres numériques utiles pour les projets développés dans leurs équipements.

Finalement, la démarche de création est plus liée à la définition du projet qu'à sa réalisation technique. C'est en cela que le numérique apporte à l'offre documentaire un plus incomparable. Le bibliothécaire réalise le document de sa conception à l'objet final et jusqu'à sa diffusion/distribution. Nous avons facilement réalisé Les enquêtes du commissaire Jérôme, de Maurice Renard ${ }^{7}$ : l'ouvrage peut maintenant être diffusé à la bibliothèque dans le cadre de l'exposition mais aussi sur son portail, sur le site de la collectivité, sur la Bibliobox, sur des bibliothèques en ligne et bien sûr

5. Intervention de Bernard Strainchamps, librairie en ligne Feedbooks et médiation sur le blog $<$ http://blog.feedbooks.com/fr/>.

6. La partie technique de la formation était assurée par Pierre-Emmanuel Malissin des éditions Syllabaire, éditeur/distributeur numérique : < http://syllabaire-editions.com/fr/>.

7. Maurice Renard, Les enquêtes du commissaire Jérôme, < http://editions-opoto.org/ >. 
circuler sur le Web par l'intermédiaire de librairies en ligne (toujours gratuitement)...

Le processus peut se répéter pour bien d'autres projets : atelier d'écriture, concours de nouvelles, compte rendu de journées professionnelles...

Les bibliothèques possédant des fonds patrimoniaux peuvent très simplement publier des textes en epub et même en retirer des royalties comme lors de l'édition d'inédits posthumes.

LES PRINCIPALES ÉTAPES DE LA FABRICATION ENCADRÉ 1 D'UN LIVRE NUMÉRIQUE

Création du texte: Word/Libre Office

Le livre se compose d'un seul fichier relu et corrigé (éventuellement avec l'utilisation d'un correcteur plus efficace que celui du traitement de texte comme Antidote, Cordial ou l'extension libre et gratuite Language Tool pour Libre Office).

Création de la structure du livre (couverture, page de titre, sommaire, chapitres, notes...)

Création de l'epub : Atlantis

< http://www.atlantiswordprocessor.com/ fr/ $>$

Atlantis est un logiciel qui permet d'enregistrer très simplement un fichier .doc ou .odt en .epub.

Vérification : EpubValidador

< http://validator.idpf.org/>

Cet outil en ligne permet de valider son fichier epub.

Modifications/réglages

(éventuellement) : Sigil

< http://code.google.com/p/sigil/>
Bien que Sigil soit un logiciel permettant de créer intégralement un livre numérique, nous l'utilisons uniquement dans le cas de petites modifications car il nécessite une bonne connaissance de HTML et CSS. Si le fichier Word est correct, Sigil n'est pas utile.

Test : Calibre ou autre bibliothèque tablette, iPad, liseuse, smartphone

< http://calibre-ebook.com/>

$<$ https://addons.mozilla.org/fr/firefox/ addon/epubreader/ >

Il est important de contrôler et tester le comportement de son livre numérique sur différents supports de façon à pouvoir éventuellement repérer quelques défauts et les corriger avant de mettre l'ouvrage en ligne.

Pour aller plus loin :

Le tutoriel Comment transformer un document électronique simple (Word ou autre) en livre numérique (EPUB, MOBI...) par JeanClaude Dunyach (Version du 10 septembre 2013) résume clairement le processus de fabrication d'un livre numérique. 


\section{LE LIVRE NUMÉRIQUE ENRICHI AU CLL : 2009-2014}

+++++++++++++++++++++++++++++++++++++++++++++++++++++++++++++++++++++++++

Il est aussi possible de réaliser des livres numériques plus complexes intégrants des fichiers images, sons et vidéo ${ }^{8}$.

\section{UNE ORIENTATION FONDS PATRIMONIAUX}

Nous avons choisi, pour illustrer cette partie, un projet de valorisation du patrimoine dans le cadre du Plan d'action pour le patrimoine écrit (PAPE).

\section{ENCADRÉ 2 LE PLAN D'ACTION POUR LE PATRIMOINE ÉCRIT}

La Direction du livre et de la lecture (aujourd'hui Service du livre et de la lecture, SLL) a engagé en 2004 un plan d'action pour le patrimoine écrit afin de mieux connaître ce patrimoine et soutenir les actions de conservation, de signalement et de valorisation menées par les collectivités territoriales qui en ont la charge.

Une enquête nationale conduite en partenariat avec les directions régionales des affaires culturelles (DRAC) a permis de dresser un état des lieux de ce patrimoine et d'identifier les principaux besoins. En Poitou-Charentes, la DRAC a confié la réalisation et le dépouillement de l'enquête PAPE à l'Agence de coopération des bibliothèques, centres de documentation et services d'archives de Poitou-Charentes (ABCD), aujourd'hui intégrée au sein du CLL.

De nombreux acteurs nationaux sont également impliqués dans le plan d'action pour le patrimoine écrit, notamment la BnF, opérateur de l'État qui mène pour son compte de nombreux programmes de conservation, de signalement ou de valorisation.

Dans le cadre du PAPE, le CLL a réalisé un inventaire régional signalant les fonds patrimoniaux conservés en Poitou-Charentes dans les bibliothèques, les services d'archives et quelques autres établissements documentaires ayant des fonds pouvant intégrer des projets de numérisation, afin :

8. Ce genre d'ouvrage numérique peut être réalisé avec le logiciel gratuit ibooks Author mais cela implique de n'utiliser que du matériel Apple, ordinateur pour la conception et iPad pour la lecture. 
- de signaler dans le Catalogue collectif de France (CCFr) les fonds patrimoniaux de Poitou-Charentes (réalisation des fiches de fonds) ;

- de repérer les fonds qu'il conviendrait de numériser et les décrire (en lien avec les programmes de numérisation) ;

- de repérer les fonds à rétroconvertir et les décrire.

Dans une perspective de valorisation de ces fonds, le CLL a missionné l'écrivain Alberto Manguel ${ }^{9}$. Il avait carte blanche pour écrire une série d'articles sur les fonds patrimoniaux de ces établissements. Seize articles ont été publiés entre 2010 et 2013 dans la revue Actualités Poitou-Charentes et sur le site Web du CLL ${ }^{10}$.

Dans le même temps, toujours dans le cadre du PAPE, le CLL confie à une équipe de documentaristes professionnelles (Les Yeux d'Yzo) la réalisation de neuf films tous publics (environ 15 minutes chacun) sur les collections patrimoniales de cinq bibliothèques et quatre services d'archives départementales. Six sont actuellement en ligne ${ }^{11}$.

Sur la même période, le CLL coordonne les campagnes de numérisation initiées par la Bnf (la collaboration débouchera en 2014 sur l'un des tout premiers portails Gallica Territoires).

Une question se pose alors : comment rassembler tous ces éléments afin de permettre à tous les équipements de les utiliser et de les rendre accessibles à un large public (les fameux 60 à $80 \%$ de la population qui ne fréquentent pas les bibliothèques) ?

Notre réponse a été la conception d'un livre numérique enrichi.

Si le projet n'a vu le jour qu'en 2012 alors que l'inventaire et le début du travail d'Alberto Manguel ont débuté en 2009, on peut dire que nous avons trouvé avec le livre numérique enrichi une solution correspondant à nos attentes de valorisation qui n'existait pas encore techniquement à l'origine du projet.

9. Résident d'un village du Poitou.

10. < http://www.livre-poitoucharentes.org/section-bibliotheque/patrimoine-ecrit/publicationspatrimoine/textesvalorisation.html >.

11. < http://www.livre-poitoucharentes.org/videos/fondspatrimoniaux.html >. 


\section{LE PROCESSUS DE CRÉATION}

L'aspect technique a été confié à la société IGS-CP, spécialisée dans le traitement du texte et la valorisation de l'image. Située près d'Angoulême, elle associe son expérience métier aux dernières technologies informatiques pour proposer des solutions productives de traitement du texte et de l'image. Un cahier des charges a défini les besoins, les solutions techniques et les attributions de chacun.

Les choix techniques permettent que le livre soit lisible sur tous supports : il est réalisé en epub3 reflowable enrichi (lisible uniquement sur iPad), puis décliné et optimisé pour tablettes et liseuses. L'epub est alors codé en amélioration progressive : sur une machine ne supportant pas les interactivités, les vidéos sont remplacées par des images ; les galeries par des suites d'images...

Le livre numérique enrichi est prévu pour être mis à jour régulièrement. Il est possible de le faire évoluer, de permettre l'ajout de nouveaux documents (les trois derniers films vidéos ne seront finalisés que fin 2014, des livres numériques peuvent être fabriqués et intégrés, des documents issus de campagnes de numérisation peuvent aussi être insérés...).

En plus des documents fournis par le CLL ou ses partenaires (photos, cartes, plans, estampes, sons, vidéos...), s'ajoute un certain nombre de documents liés à la valorisation du patrimoine écrit (et pas seulement) picto-charenteais comme les éditions numériques lorsqu'elles existent, des ouvrages mentionnés par Alberto Manguel, ou des documents d'autres ouvrages ${ }^{12}$. Il est bien sûr tout à fait possible de réaliser un livre numérique à partir d'un document qui n'existe pas au format epub mais qui est déjà numérisé (par la BnF ou par un partenaire). On procède alors comme explicité plus haut pour le livre numérique de Maurice Renard. Il suffit ensuite de l'intégrer dans notre livre numérique enrichi.

Le livre que nous avons inventé collectivement autour des textes d'Alberto Manguel ressemble finalement à une bibliothèque. Parce qu'il est enrichi, ce livre propose différents supports choisis, ordonnés et pensés comme lors de la constitution des collections d'une nouvelle bibliothèque. Une

12. Ce fut le cas, par exemple, pour les photographies d'Aurélie Pétrel qui accompagnent le texte de François Bon sur la médiathèque de Poitiers, " De haut en bas », in Tours et détours en bibliothèque, Carnet de voyage, Villeurbanne, Presses de l'enssib, 2012 (coll. enssib 2012), pp. 126-143. 
bibliothèque certes particulière, plutôt modeste, mais dont l'ensemble des titres qui la composent est valorisé ${ }^{13}$.

Les outils disponibles aujourd'hui, et cela sera encore plus vrai demain, ouvrent des perspectives inédites pour les bibliothécaires qui peuvent, au-delà de leurs pratiques éditoriales souvent contraintes financièrement ou épisodiques, développer une politique éditoriale liée aux collections. Cela est valable pour toutes les bibliothèques : de la bibliothèque rurale associative à la BMVR.

De projets modestes réalisés par les bibliothécaires à la création plus complexe de livres enrichis, l'utilisation du numérique permet aujourd'hui de rendre la bibliothèque plus autonome, plus actrice de ses projets et de se positionner dans un espace d'édition pour la valorisation de ses collections et la médiation de la lecture.

C'est une autre facette du métier de bibliothécaire qui se profile à l'horizon : le numérique (ici sans distinction entre livre numérique et livre numérique enrichi) permet de s'affranchir de certaines tâches (le catalogage par exemple) pour se consacrer à des missions plus proches des usagers présents et futurs, pour explorer d'autres outils de médiation, pour finalement tendre vers la vision de Jack London : " sur les rayons des bibliothèques je vis un monde surgir de l'horizon".

13. Voyage de mémoire : promenades et découvertes dans les bibliothèques et archives de PoitouCharentes est finalisé et disponible gratuitement sur le site du CLL < http://www.livre-poitoucharentes.org/ > et dans Appstore. 


\section{PARTIE IV}

\section{LES OUTILS ET LE DROIT}

1. PRODUCTION DE CONTENUS EN LIGNE : MISES EN GARDE JURIDIQUES par Michèle Battisti

$++++++++++++++++++++++++++++++++++++++++++++++++++++++$

2. ÉCRIRE POUR LE WEB

par Isabelle Rouquet

t + + + + + + + + + + + + + + + + + + + + + + + + + + + + + + + + + + + + + + + + + + + + + + + + + + +

3. PANORAMA DES OUTILS DE PRODUCTION EN LIGNE par Lionel Dujol 


\section{1}

PRODUCTION DE CONTENUS

EN LIGNE : MISES EN

Michèle

GARDE JURIDIQUES

Battisti

Votre projet est bouclé : votre site sera alimenté par un large éventail de contenus propres à séduire un public très varié. Oui, mais de quels droits disposez-vous pour ceci ? N’y aurait-il pas des négociations à envisager ? Avec qui ? Quels risques prenez-vous lorsque les zones juridiques sont grises?

\section{QUELS DROITS ?}

Pour déterminer les droits à prendre en compte, on classera les types de contenus mis à la disposition de son public. On distinguera ainsi :

- des documents primaires, soit généralement des œuvres ;

- des documents secondaires, résultat d'un traitement documentaire descriptif ;

- des documents tertiaires*, produits documentaires plus élaborés, formes condensées d'un contenu.

Mais les sommaires, prémières et quatrièmes de couverture des publications, par exemple, utilisés à des fins documentaires, ne sont-ils pas des (éléments de) documents primaires ? Les commentaires des internautes ne sont-ils pas des documents tertiaires d'un autre type ? Le paysage se trouble et on perdra, dès à présent, l'idée de tout maîtriser.

Un paysage complexe n'évite pas pour autant de se mettre en règle avec le droit. On poursuivra donc le travail à mener en amont en se posant d'autres questions : 
- Les contenus sont-ils proposés par des éditeurs ? Sont-ils créés par vous-même ? Par des collègues de votre établissement ? Par des personnes extérieures?

- Les documents proviennent-ils des fonds de votre établissement ? Ont-ils fait l'objet d'une négociation contractuelle ? Laquelle ? Etc.

Penchons-nous à présent sur le droit d'auteur, la branche du droit essentielle en la matière.

\section{L'GEUVRE EST-ELLE PROTÉGÉE PAR LE DROIT D'AUTEUR ?}

Puisque le droit d'auteur protège toutes les créations intellectuelles dont la mise en forme est originale, il faut déterminer si les documents utilisés " portent l'empreinte de la personnalité » de leurs auteurs. Sachant que même un descriptif de brevet, soit une notice très technique, répond généralement à ce critère, il est prudent d'imaginer que l'œuvre que l'on veut utiliser est plutôt protégée par le droit d'auteur.

\section{Le domaine public?}

Mais la protection peut avoir expiré. L'œuvre n'est-elle pas dans le domaine public 70 ans après la mort de l'auteur ? Encore faut-il réussir à définir précisément si c'est le cas. Années de guerre, auteurs morts pour la France, œuvres de collaboration, œuvres collectives, anonymes et pseudonymes, œuvres posthumes, autant de situations à prendre en compte. Autant de difficultés qu'entend résoudre un calculateur du domaine public ${ }^{1}$ et que souligne le rôle clef joué par les métadonnées culturelles (nom de l'auteur, nature de l'œuvre, qualification et statut juridique de l'auteur, etc.). Vous êtes certain que tous les éléments (enrichissements, traductions, etc.) de l'œuvre appartiennent au domaine public ? Si c'est le cas, n'oubliez pas cependant de respecter les droits moraux de l'auteur, notamment l'obligation de mentionner le nom de l'auteur et, plus subjectif, de respecter l'intégrité de l'œuvre utilisée. Les 
droits moraux, attachés à la personnalité de l'auteur, auxquels l'auteur ne peut jamais renoncer, sont perpétuels, exercés après sa mort par ses héritiers.

L'œuvre est encore sous droit (ou vous n'êtes pas sûr qu'elle soit dans le domaine public). Mais ne peut-on faire valoir des exceptions au droit patrimonial de l'auteur, soit aux droits de reproduction et de représentation dont l'auteur a le contrôle ? S'il s'agit d'alimenter un site Web, aucune ouverture, ou si peu. Oublions, en effet, ici : les usages privés (copies privées et représentations dans le cercle dit « de famille ») ; les usages réservés à un public précis de l'exception pédagogique et de recherche et de l'exception au bénéfice de personnes handicapées ; la communication sur place autorisée par l'exception accordée (pour certaines œuvres) aux bibliothèques « accessibles au public ». Reste l'exception pour parodie, la caricature et le pastiche, soit un exercice très particulier, tout comme l'exception accordée aux revues de presse et la reprise de discours publics liés à l'actualité. Quant à la citation, courte, elle doit alimenter une réflexion et ne peut pas (en théorie, car les outils de curation du Web 2.0 bousculent cette affirmation) s'appliquer à une liste d'extraits, encore moins à la reprise d'une image que l'on entend commenter.

L'œuvre que vous souhaitez numériser fait partie de votre fonds. Certes, mais vous n'êtes généralement propriétaire que du support et ne disposez pas des droits vous permettant d'exploiter l'œuvre qui y figure sous cette nouvelle forme ${ }^{2}$. Vous ne retrouvez pas un auteur ? Que faire alors de cette œuvre orpheline de droits ? Il est temps, à présent, de se pencher sur la gestion des droits.

2. Si l'œuvre originale (et non sa copie) qui est dans votre fonds n'a pas été publiée et que la période post-mortem de 70 ans est échue. Dans ce cas, votre institution dispose des droits pendant 25 ans après la communication de l'œuvre. 


\section{QUE DOIT-ON NÉGOCIER ?}

\section{QUI A LES DROITS ? L'AUTEUR, VOTRE ÉTABLISSEMENT, OU DES TIERS ?}

Savoir auprès de qui négocier, voici la question à se poser ensuite. Les collègues de votre établissement, auteurs de certaines contributions, ont-ils cédé les droits à leur employeur ? Si vous opérez dans le secteur public, la création fait-elle partie de leur mission? Si ce n'est pas le cas, bien que peu d'employeurs (et d'employés) le savent, ce sont vos collègues qu'il conviendrait de contacter. Cependant, si l'œuvre de votre collègue a été publiée par un éditeur ou un producteur, des conditions contractuelles pourraient faire obstacle à toute réutilisation. Il convient de vérifier que l'usage voulu ne leur a pas été déjà cédé de manière exclusive à l'éditeur ou au producteur et, si ce devait être le cas, renégocier les droits pour disposer de cet usage. Vous faites appel à des tiers : ce sont les éditeurs, les producteurs ou les auteurs eux-mêmes (par exemple dans le cadre de la commande d'un produit tel qu'un «panorama de presse » ou, plus classiquement pour une photographie, un texte, etc.) qu'il convient de contacter et auxquels vous serez lié par un contrat prévoyant la cession des droits pour les usages envisagés de l'œuvre. Attention ! Tout usage non prévu au départ doit faire l'objet d'un avenant au contrat initial sous peine de rencontrer de sérieuses difficultés. Toute mention vague, comme une cession « tous droits inclus » ${ }^{3}$ ou " pour tous usages » est à éviter.

Des contrats avec les sociétés de gestion collective ? S'il s'agit de musique, la Société des auteurs, compositeurs et éditeurs de musique (SACEM) est généralement incontournable. Pour l'image, on peut s'adresser à la Société des auteurs dans les arts graphiques et plastiques (ADAGP), la Société des auteurs des arts visuels et de l'image fixe (SAIF), etc. Mais s'il s'agit de textes, dans l'environnement numérique, le Centre français d'exploitation du droit de copie (CFC) ne peut vous proposer (si vous le souhaitez, car

3. Joëlle Verbrugge, "Pack-shot, originalité et nullité d'une clause imprécise ", in Droit et photographie, 23 décembre 2013. [En ligne] : < http://blog.droit-et-photographie.com/pack-shot-originalite-et-nullite-dune-clause-imprecise/ > 
la loi ne l'impose pas) que des contrats pour des panoramas de presse sur intranet, sur extranet ou des copies numériques internes. Quant aux usages pédagogiques et de recherche, couverts au titre de l'exception pédagogique, pour certaines œuvres, ils ne s'appliquent qu'à certains usages destinés majoritairement à des étudiants ou des chercheurs, en classe, pour des sujets d'examens ou lors de congrès ou séminaires. Rien de pertinent pour un site ouvert à tous, même si ceux-ci devraient être réservés aux usagers inscrits à votre bibliothèque ou centre de documentation. Livres, périodiques... des autorisations expresses avec les éditeurs doivent être envisagées. Et celles-ci doivent pouvoir être produites - donc remises par écrit - lors de toute contestation. Garder les traces des autorisations données s’impose.

\section{Les œuvres orphelines}

Restent les œuvres orphelines, ces œuvres sous droit ou qui pourraient l'être car on peine à trouver leurs auteurs ou ayants droit pour négocier les droits nécessaires. Lorsqu'il s'agit de livres « indisponibles », des livres publiés avant le $1^{\mathrm{er}}$ janvier 2001 qui ne sont plus commercialisés, c'est la Société française des intérêts des auteurs de l'écrit (SOFIA), une autre société de gestion collective qui serait votre interlocuteur. Pour les autres œuvres - périodiques, presse, illustrations, musique, vidéos - une loi transposera en France une directive européenne du 25 octobre 2012. Certains établissements accessibles au public pourront, sous certaines conditions, notamment après une recherche « diligente » des ayants droit, mettre ces œuvres à la disposition d'un public.

Les boutons de partage figurant sur bon nombre d'articles ou de billets de blogs vous permettent d'alerter votre public. Oui, mais ce sont de courts extraits, munis quelquefois de la photographie qui les accompagnent qui sont ainsi reproduits sur Facebook, LinkedIn, etc. via des outils du réseau social tels que Diigo, Scoop.it, etc. et ce, bien que les conditions générales d'utilisation (CGU) des sites interdisent (généralement) toute rediffusion partielle du contenu du site. Tant que vous ne mettez pas à la disposition l'article dans son intégralité, lorsque celui-ci n'est plus accessible qu'aux abonnés, pourquoi pas ? Ne vous bornez-vous pas à aiguiller votre public 
sur des contenus auxquels il a de toute manière accès ? ${ }^{4}$ Voilà une superbe zone grise : le titre de presse bénéficie de lecteurs supplémentaires, mais vous-même vous tirez parti d'un service que vous proposez (même si celui-ci est gratuit).

Bien mieux, certains sites - Fotopedia, YouTube, etc. - permettent d'incruster les œuvres que l'on y trouve sur votre propre site. Si d'aventure elles disparaissaient sur le site initial après notification des ayants droit ayant signalé leur présence illicite, elles disparaîtraient automatiquement de votre site. Voilà qui limite votre propre responsabilité. Les noms des auteurs et les sources des œuvres incrustées sur votre site doivent cependant être correctement mentionnés, sous peine d'être accusé de parasitisme pour s'être approprié indûment l'œuvre d'autrui.

\section{Les œuvres " libres de droit »}

Certaines œuvres sont « libres de droit ». Certes, méfiez-vous toutefois de cette appellation trompeuse. Elles ne sont « libres » que dans les limites des usages définis par l'auteur de l'œuvre. Des conditions sont imposées (une éventuelle interdiction de la modifier, d'en faire un usage commercial, de modifier les conditions contractuelles définies initialement par l'auteur, etc.) pour utiliser les œuvres récupérées (souvent achetées) dans des banques d'images ou celles, pour prendre un autre exemple, qui sont accompagnées d'une des licences Creative Commons ${ }^{5}$. Attention! Libre accès ne signifie pas toujours libre réutilisation. Sur Flickr, par exemple, la majorité des photographies (6 milliards en 2011) sont accompagnées du symbole (c). Toute utilisation implique une demande expresse. Pas de (c) apposée à une œuvre ? Originale, elle n'en reste pas moins protégée et le droit d'auteur s'y applique avec la même force. Une autorisation expresse pour tout usage souhaité s'impose tout autant.

\footnotetext{
4. Le lien est libre s'il donne accès à des contenus librement accessibles, Arrêt de la Cour de justice de l’Union européenne (affaire C 466/12) du 13 février 2014.

5. < http://creativecommons.fr/ >.
} 


\section{QUELLE RESPONSABILITÉ ?}

\section{ÉDITEUR OU HÉBERGEUR ?}

Telle est la question, car les règles ne sont pas les mêmes.

- Responsabilité a priori pour l'éditeur, responsable d'emblée pour toutes les informations illicites qu'il diffuse. Il peut s'agir d'œuvres contrefaisantes, bien sûr, mais aussi des atteintes à la vie privée, au droit à l'image, ou pour délits de presse, soit diverses incitations à la haine ou à la violence commises par voie de publication ;

- Responsabilité a posteriori pour l'hébergeur, responsable pour les informations manifestement illicites ou, après avoir été informé de l'existence sur son site d'une information illicite, s'il ne les a pas retirées " promptement ». Mais si votre site est modéré, ce qui suppose un contrôle a priori des contenus, vous ne pourriez plus vous appuyer sur cette disposition qui limite votre responsabilité qui vous évitait même aujourd'hui ${ }^{6}$ de vérifier que toute œuvre signalée une première fois n’a pas réapparu sur votre site.

\section{RESPONSABILITÉ CIVILE ET PÉNALE}

Responsabilité civile pour les préjudices causés à des tiers par sa faute ou, tout simplement, par sa négligence. Vous êtes intrigué ? Les informations diffusées peuvent fort bien être fausses, incomplètes, obsolètes, secrètes et utilisées par des tiers, avoir des conséquences graves. Il suffit de se placer dans un contexte médical, financier, industriel, etc. La faute est intentionnelle ? Une responsabilité pénale (amendes et peine de prison) sera encourue.

6. La fin du système "notice and stay down" et le retour au système du "notice and take down", Jacob Avovats, 30 juillet 2012. [En ligne] : < http://www.jacobavocats.com/la-fin-du-systeme-denotice-and-stay-down-et-le-retour-au-systeme-du-notice-and-take-down/ >. 
Une clause de non-responsabilité : votre profil professionnel figure dans les mentions légales de votre site ? N'imaginez pas pour autant que cela vous donne un blanc-seing et évite toute condamnation. Un juge peut toujours évaluer votre responsabilité en fonction de votre profil et d'un contexte donné. On rappellera, bien sûr, que c'est votre employeur qui répondra le plus souvent ${ }^{7}$ des fautes de son salarié et qui pourrait se retourner contre vous ensuite.

\section{QUEL PAYSAGE JURIDIQUE ?}

\section{UNE SITUATION APPELÉE À ÉVOLUER}

C'est le droit, mais aussi les contrats qui peuvent avoir été conclus qui vous imposent les règles à respecter. Non seulement celles-ci varient ainsi selon les établissements, mais la loi elle-même pourrait changer. Une loi sur les livres indisponibles récemment, une autre sur les œuvres orphelines prochainement, mais aussi une directive européenne sur le droit d'auteur et les droits voisins qui serait révisée, modifiant ainsi la loi française, etc. Verra-t-on apparaître une exception au droit d'auteur pour réaliser des œuvres transformatives et les diffuser dans un cadre non marchand, voire, pourquoi pas, un élargissement de l'exception accordée à la citation, pour ne prendre que deux exemples ? Sera-t-on plutôt face à une gamme de licences simples à utiliser qui, moyennant un prix raisonnable, ouvrira l'éventail des œuvres utilisables ? L'un ou l'autre, l'un et l'autre ?... Il est nécessaire de maintenir une veille sur les questions juridiques.

\section{LES ZONES SONT GRISES}

Éviter de s'y glisser, sous peine de limiter considérablement l'accès à l'information, au dam même des ayants droit ? Ne peut-on pas imaginer faire valoir un droit distinct pour des usages informatifs (à l'image des titres d'œuvres que l'on utilise dans les notices bibliographiques, même si, originaux, ils sont protégés par le droit d'auteur, ou pour les logos, bien que

7. Muriel Cahen, La responsabilité de l'employeur du fait de l'activité de ses salariés, Net-Iris, 8 juin 2007. 
souvent protégés par le droit des marques, lorsqu'ils sont utilisés à des fins d'information) permettant la reprise de couvertures de publications, de quatrièmes de couverture, la reprise de résumés... car « lieux sans âge, lieux de mémoire, les centres de documentation sont des sanctuaires, et l'information que l'on y trouve ne devrait pas être la cible de multiples couches juridiques définies pour d'autres cadres. L'information est polymorphe et les règles de droit qui peuvent exploser à la tête des documentalistes sont multiples. L'information qui y est traitée doit être détachée du contexte social où elle était produite. Il faut concevoir un droit spécifique qui protège ceux qui manipulent l'information et la mettent à la disposition d'un public dans un contexte différent du contexte initial. ${ }^{8}$

8. Cédric Manara, «Droit de l'information : paradoxes et ouvertures », Documentaliste-Sciences de l'information, 2012, volume 49, $\mathrm{n}^{\circ} 3$, pp. 18-19. 


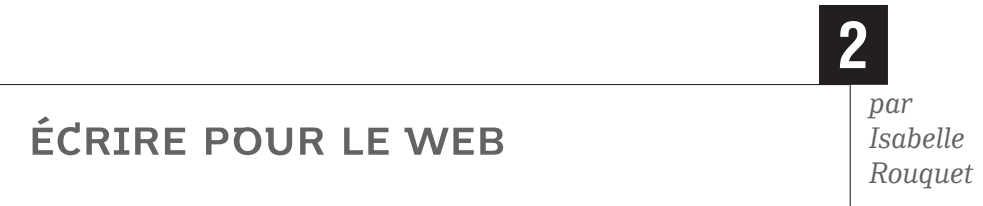

Aujourd'hui, le bibliothécaire est amené à produire des contenus rédactionnels adaptés au Web afin de les mettre en relation avec des internautes. "Dans l'industrie du Web, est appelé « contenu » tout ce qui véhicule de l'information porteuse de sens pour l'être humain. Tous les sites Web ont du contenu. $»^{1}$

Commençons par situer la rédaction Web dans le cadre plus global de la stratégie de contenus. Comme le soulignent Kristina Halvorson et Mélissa Rach, " la stratégie de contenu régit la création, la publication et l'entretien de contenus utiles et utilisables ». Travailler le cycle de vie des contenus $\mathrm{Web}^{2}$ aide les bibliothécaires à comprendre et à produire les types de contenus dont leur public-cible a réellement besoin.

Le cycle de création de contenu fait référence à la création du document par un rédacteur. Il comprend l'analyse des besoins, la récolte de l'information, la structuration, la mise en texte, l'édition, la correction et la mise en forme. Selon les bibliothèques, le rédacteur peut être son propre éditeur ; mais on trouvera également des "valideurs », des " secrétaires de rédaction » ou encore des " coordinateurs » chargés notamment d'aider les rédacteurs et de vérifier la bonne conformité des publications avec la charte éditoriale.

La mise en ligne consiste à rédiger le texte dans l'outil d'aide à la publication de contenu en place au sein de la bibliothèque, appelé CMS.

La première partie de cet article, intitulée Les choix du rédacteur, détaille la réflexion indispensable à mener en amont de la rédaction d'un contenu. La seconde partie, intitulée L'écriture efficace, cible la rédaction ellemême et met en avant les contraintes rédactionnelles. La dernière partie,

1. Erin Kissane, Stratégie de contenu web, Paris, Eyrolles, 2011 (coll. A Book Apart, 3).

2. Eve Demange, « Le cycle de vie du contenu web », Blog Plume interactive, novembre 2013. [En ligne] : < http://plumeinteractive.canalblog.com/archives/2013/11/22/28487716.html >. 
intitulée La mise en ligne, insiste sur les principales techniques visant à optimiser l'efficacité du référencement des pages lors de la mise en ligne.

\section{LES CHOIX DU RÉDACTEUR}

Le rédacteur ne cède pas à la tentation de commencer à écrire tout en réfléchissant, au fur et à mesure, aux divers éléments de planification de son contenu.

\section{LE CHOIX D'UN ANGLE ÉDITORIAL}

L'angle est la «manière d'aborder un sujet, de le ramener à un aspect principal bien délimité »3. Pour un sujet donné, le rédacteur doit chercher comment traiter son sujet, trouver une approche originale. La recherche d'angle est le fruit du travail et de la créativité du rédacteur. Elle vise à capter l'attention du public, à surprendre par un contenu attractif et original.

Produire un contenu sur le sujet des vampires pousserait le rédacteur à écrire un article fourre-tout qui aborderait tous les aspects du sujet. La conséquence serait inévitablement de noyer son public et de le perdre. Il convient de donner un cadre à cette production en fonction de l'internaute auquel on s'adresse. Il existe plusieurs techniques pour définir un angle. La technique du « camembert », par exemple, décrite par Christian Robin ${ }^{4}$ consiste à inscrire son sujet au centre du camembert, puis à lister tout autour les acteurs concernés par ce sujet. Ces acteurs représentent chaque segment de notre camembert et peuvent être un individu précis, un corps de métier, une institution, une catégorie sociale ou familiale, etc. Le rédacteur tente de formuler toutes les questions que se pose cet acteur sur le sujet, et génère ainsi une multitude d'angles possibles.

Par exemple, les passionnés de littérature de vampires s'interrogeront sur la question suivante : "Pourquoi rencontre-t-on le vampire dans toutes les civilisations et à toutes les époques ? » Les médecins soulèveront peutêtre la question suivante : «quelles sont les maladies expliquant le mythe

3. Yves Agnès, Manuel de journalisme, Paris, La Découverte, 2008 (coll. Grands Repères, Guides).

4. Christian Robin, L'angle journalistique : techniques de créativité pour des écrits originaux, Paris, CFPJ Éditions, 2009. 
du vampire ? » Le rédacteur met en avant dans ce cas les travaux de certains médecins ayant fait le parallèle entre les signes caractéristiques des vampires et des symptômes de certaines maladies.

La recherche d'angle n'est pas une étape aisée, et reste difficile pour les rédacteurs occasionnels. "Porter enfin un regard critique sur ce que nous écrivons afin d'améliorer ce que nous offrons à nos lecteurs. Développer sa créativité est une gymnastique quotidienne, un état d'esprit permanent [...]. » (Christian Robin) ${ }^{5}$.

Cette étape est particulièrement importante pour ce qui concerne les marronniers, ces articles sur un événement qui revient chaque année à date fixe. Écrire sur le Salon du Livre, c'est intéressant, encore faut-il donner un angle à l'article pour ne pas tomber dans la production d'articles généraux sur le sujet.

\section{LE CHOIX DES INFORMATIONS}

Il permet au rédacteur de ne garder que ce qui est utile à sa production selon le point de vue choisi.

\section{LE CHOIX DU GENRE}

Les sites Web des bibliothèques accueillent diverses formes de contenus de médiation numérique. Certaines formes de contenus font appel à l'écriture ; ce sont des genres journalistiques, soit les différentes manières de rédiger un article. Le rédacteur doit connaître les caractéristiques de chaque genre dans lequel il est susceptible d'écrire.

Par exemple, le rédacteur qui écrit une brève doit savoir que c'est un article d'information stricte formé d'un seul paragraphe, qu'il s'agit du genre le plus court qui énonce en peu de mots des réponses aux questions de référence. À l'inverse, l'écriture d'une critique (article de commentaire), nécessite d'apporter des éléments d'information stricte sur le document acquis par la médiathèque, une part de jugement et, si possible, une part objective née de la compétence du bibliothécaire.

5. Christian Robin, op. cit. 


\section{LE CHOIX D'UNE LONGUEUR ADAPTÉE}

La rédaction ne faisant pas partie, pour de nombreux bibliothécaires, de leur métier d'origine, il est conseillé d'inclure dans la charte éditoriale des recommandations précises sur la granularité de l'information et donc d'indiquer une fourchette sur le nombre de signes autorisés pour les différents genres produits par la bibliothèque.

\section{CHOISIR LA STRUCTURATION DES INFORMATIONS}

Avant d'écrire, le rédacteur choisit le plan de son article. Cette réflexion lui permet de connaître la construction logique de son article et l'enchaînement des paragraphes. Le plan recommandé sur le Web est celui de la pyramide inversée : c'est un plan journalistique " consistant à développer l'information essentielle donnée dans le premier paragraphe par d'autres informations classées par ordre décroissant d'importance $»^{6}$. Le premier paragraphe développe donc le message essentiel.

\section{LE CHOIX DU MESSAGE ESSENTIEL}

L'internaute ne retient d'un texte qu'une seule idée dominante quelle que soit la longueur du texte. Le rédacteur organise donc sa production autour d'un message essentiel que l'on peut définir ainsi : " Fait ou idée centrale d'un article, autour duquel s'organise celui-ci. » ${ }^{7}$. Le choix du message essentiel découle de l'angle. Cette idée dominante apparaît dès le titre et le début de l'article et garantit une bonne compréhension de l'ensemble de la production par l'internaute.

Ce message est constitué par la réponse aux questions de référence : qui, quoi, où, quand, comment, pourquoi. Il s'agit des $5 \mathrm{~W}+\mathrm{H}^{*}$, formulation anglo-saxonne de Who, What, Where, When, Why + How.

Le rédacteur s'assure qu'à travers le message essentiel, la communication remplit bien les objectifs établis dans la ligne éditoriale.

6. Yves Agnès, op. cit.

7. Yves Agnès, op. cit., p. 103. 


\section{LE CHOIX DU TON}

Il dépend de la ligne éditoriale du site Web de la bibliothèque qui sera formalisée dans la charte éditoriale. Comme l'énonce Yves Agnès, le ton pourra osciller : « De la plus grande neutralité à la complicité avec le lecteur, d'une écriture sobre à une écriture plus littéraire, au vocabulaire plus recherché, d'un « style institutionnel » à un style plus concret, plus vivant... ».

On note l'importance pour une bibliothèque de réfléchir à sa ligne éditoriale, car celle-ci définit le fil rouge de la communication utile aux rédacteurs. Elle définit les éléments suivants : l'objectif de la communication, les cibles, les thématiques abordées, le rythme de publication et le style rédactionnel.

\section{LE CHOIX DES MOTS-CLÉS : RÉFÉRENCEMENT ET STRATÉGIE DE VISIBILITÉ}

Les pages de contenu doivent être optimisées en fonction des critères de pertinence des moteurs. Elles seront ainsi classées au mieux dans les pages de résultats des liens organiques lorsque les mots-clés importants pour l'activité de la bibliothèque sont saisis par les internautes.

Le rédacteur établit donc une liste de mots-clés ou expressions mots-clés. Il fait appel tout d'abord à son intuition et a recours à certains services permettant de rechercher des termes pertinents à partir de termes de départs. Citons notamment Le grand dictionnaire terminologique ${ }^{8}$, le Dictionnaire électronique des synonymes ${ }^{9}$, le Trésor de la langue française informatisé (TLFI) ${ }^{10}$, ou encore la liste d'autorité Rameau.

Les rédacteurs les plus chevronnés peuvent aller plus loin dans ce choix des mots-clés et trouver un arbitrage entre le potentiel des termes choisis ou l'intérêt et la faisabilité technique d'un positionnement sur ceux-ci. Ils interrogent l'outil de planification des mots-clés de Google ${ }^{11}$ qui indique le nombre moyen de recherches mensuelles de chaque requête.

\footnotetext{
8. < http://www.oqlf.gouv.qc.ca/ressources/gdt.html >.

9. < http://www.crisco.unicaen.fr/des >

10. $<$ http://atilf.atilf.fr $>$.

11. < https://adwords.google.com/ko/KeywordPlanner >.
} 


\section{VÉRIFICATION DE L'INTÉRÊT OU POTENTIEL DU MOT-CLÉ DANS L'OUTIL DE CAPTURE D'ÉCRAN 1 PLANIFICATION DES MOTS-CLÉS GOOGLE}

\begin{tabular}{|c|c|c|c|c|}
\hline \multirow{2}{*}{$\begin{array}{l}\text { Outil de planification des } \\
\text { mots clés }\end{array}$} & \multicolumn{4}{|l|}{ Votre produit ou service } \\
\hline & \multicolumn{4}{|c|}{ orque, épaulard, baleine tueuse, mammifère marin, globicéphale } \\
\hline \multicolumn{5}{|l|}{ Ajouter des idées à votre plan } \\
\hline Ciblage? & Idées de groupes d'annonces & Idées de mots clés & & \\
\hline France & & & & \\
\hline Français & \multicolumn{2}{|l|}{ Termes de recherche } & & $\begin{array}{l}\text { herches } \\
\text { elles }\end{array}$ \\
\hline Google & \multirow{2}{*}{ orque } & & & 18100 \\
\hline Mots clés à exclure & & & & \\
\hline Personnaliser votre recherche ? & \multicolumn{2}{|l|}{ mammifère marin } & $\underline{L}$ & 1600 \\
\hline Filtres de mots clés & \multicolumn{2}{|l|}{ épaulard } & $\underline{L}$ & 880 \\
\hline $\begin{array}{l}\text { Nombre moy. de recherches } \\
\text { mensuelles } \geq 0 \\
\text { Enchère suggérée } \geq 0,00 €\end{array}$ & \multicolumn{2}{|l|}{ globicéphale } & $\underline{L}$ & 880 \\
\hline Taux dimpr. des annonces z & \multicolumn{2}{|l|}{ baleine tueuse } & $\underline{\underline{L}}$ & 320 \\
\hline
\end{tabular}

VÉRIFICATION DE LA CONCURRENCE DU MOTCAPTURE D'ÉCRAN 2 CLÉ DANS LE MOTEUR DE RECHERCHE GOOGLE

\begin{tabular}{|c|c|c|c|c|c|c|c|}
\hline \multirow{2}{*}{ Google } & \multicolumn{6}{|c|}{ orque } & $Q$ \\
\hline & Web & Images & Maps & Shopping & Actualités & Plus - & Outils de recherch \\
\hline & Envir & 451000 & ultats & 38 seconde & & & \\
\hline
\end{tabular}


Ces rédacteurs mesurent la faisabilité technique du positionnement en saisissant chaque mot concerné dans le moteur de recherche et en vérifiant le nombre de résultats.

D'une part, mieux vaut viser des expressions plus précises et avoir une concurrence moins forte. D'autre part, " il ne sert à rien d'être bien positionné sur des mots-clés que personne ne saisit » (Olivier Andrieu) ${ }^{12}$. Un tableau récapitulatif attribuant une note globale sur l'intérêt et la faisabilité permet de savoir quels mots-clés sont à traiter en priorité.

Il est important de comprendre que le concept de longue traîne* introduit par Chris Anderson peut être appliqué au monde du référencement. La queue de la longue traîne est l'ensemble des mots-clés qui, pris individuellement, génèrent peu de trafic mais qui, une fois cumulés, peuvent peser jusqu'à $80 \%$ du trafic. C'est donc le contenu textuel qui va nourrir la queue de la longue traîne. Le rédacteur choisit donc les mots-clés et écrit son contenu éditorial en fonction des publics mais aussi en tenant compte des contraintes des moteurs.

\section{L'ÉCRITURE EFFICACE}

\section{UN TEXTE À FORT IMPACT}

Comme le mentionne François Richaudeau, « un texte efficace est un texte qui permet une lecture efficace. [...]. Et, la finalité première de cette lecture efficace, c'est de marquer le lecteur, de lui imprimer une information nouvelle. [...] Un texte efficace est donc un texte qui convainc le lecteur ». Le rédacteur Web nourrit donc ses écrits d'arguments et n'écrit pas pour ne rien dire. Il écrit du contenu original et attractif ; il produit des contenus apportant une valeur ajoutée réelle à son audience. 


\section{DES TEXTES HUMAINS}

"Le rédacteur recourra à des mots et à des constructions ayant une résonance personnelle. ${ }^{13}$ Le contenu s'adressera directement au lecteur et aura recours autant que possible aux lois de proximité. « Ne laissez pas au lecteur le soin de faire la connexion entre leurs besoins et ce que vous ou votre organisation pouvez offrir. ${ }^{14}$. On préfère le " vous » à " la bibliothèque ", et on prend soin de toujours mettre en avant le pourquoi dans son contenu, c'est-à-dire les bénéfices pour les cibles ${ }^{15}$.

\section{DES TEXTES LISIBLES}

Il s'agit tant de la lisibilité visuelle que de la lisibilité rédactionnelle permettant une facilité de compréhension des mots et des phrases.

Le rédacteur prend soin d'immédiatiser l'accès à son contenu en structurant et en aérant son texte. Les accélérateurs de lecture sont par exemple un titre, des paragraphes aérés (avec une seule idée par paragraphe), des listes à puces, des mots-clés en gras, des liens hypertextes ou encore du multimédia. Offrir un accès rapide à son contenu est primordial sur le Web, espace dans lequel l'internaute scanne la page du regard à la recherche de l'information qui l'intéresse ${ }^{16}$.

Le texte n'est pas justifié, et un sommaire interactif et des intertitres complètent les textes plus longs.

La lisibilité rédactionnelle a trait au type de vocabulaire employé et à la construction des phrases. Le rédacteur utilise un vocabulaire adapté à ses lecteurs, ancré dans le langage courant. Par exemple, il évite de recourir au jargon professionnel compréhensible uniquement entre bibliothécaires. Les termes incunables, protocole Z3950 ou encore Opac ne sont pas intelligibles par tous nos lecteurs.

13. Yves Agnès, op. cit.

14. < http://s.billard.free.fr/referencement/?2006/05/24/246-7-transformations-pour-destextes-a-fort-impact $>$.

15. Lire à ce sujet : < http://www.nngroup.com/articles/user-centric-language >.

16. < http://www.poynterextra.org/eyetrack2004 >. 


\section{DES TEXTES CONCIS}

Il s'agit d'un écrit réduit à l'essentiel et exprimé en peu de mots (selon le TLFI). Chaque phrase apporte une ou des informations. Chaque mot, précis et juste, est choisi pour véhiculer au mieux cette information. Rien n'est inutile.

Si la concision est un atout pour une production papier, pour le Web c'est une véritable nécessité. On pourra toutefois écrire des textes longs sur le Web tout en respectant cette consigne.

\section{LA MISE EN LIGNE}

Parmi les techniques permettant d'optimiser l'efficacité du référencement des pages d'un site, le rédacteur place les mots-clés choisis dans les zones chaudes des pages Web. Ces zones décrites ci-dessous, permettent aux pages d'être plus réactives aux critères de pertinence des moteurs. Il prend ainsi en compte les critères in page ${ }^{17}$ lorsqu'il entre son contenu dans le CMS de sa bibliothèque. La bibliothèque a tout intérêt à expliquer aux rédacteurs l'importance de chaque champ du CMS, soit dans un document synthétique, soit directement au sein du CMS à la fin de chaque champ.

\section{SOIGNER LE TITRE DE LA PAGE}

La balise < title > correspond au titre de la page, au sens HTML du terme. Son libellé doit utiliser des mots-clés déterminants et caractéristiques du contenu de la page. Le contenu de cette balise est repris par les moteurs dans leurs pages de résultats. Un internaute qui trouve la page parmi les résultats d'un moteur est immédiatement informé sur le contenu de la page. Chaque page doit avoir un titre entre 7 et 10 mots (hors mots vides) avec la structure optimisée suivante $:<$ title $>$ [Contenu h1] - [Rubrique] [Nom du site] </title >, où h1 est le titre éditorial au sein de la page elle-même. Dans l'idéal, le rédacteur titre son contenu (h1) et ce titre est repris dans la balise $<$ title $>$.

17. Critères in page ou in the page, c'est-à-dire concernant l'analyse par les moteurs du code HTML des documents. 
CAPTURE D'ÉCRAN 3 REPRISE DE LA BALISE < TITLE > PAR LE MOTEUR GOOGLE

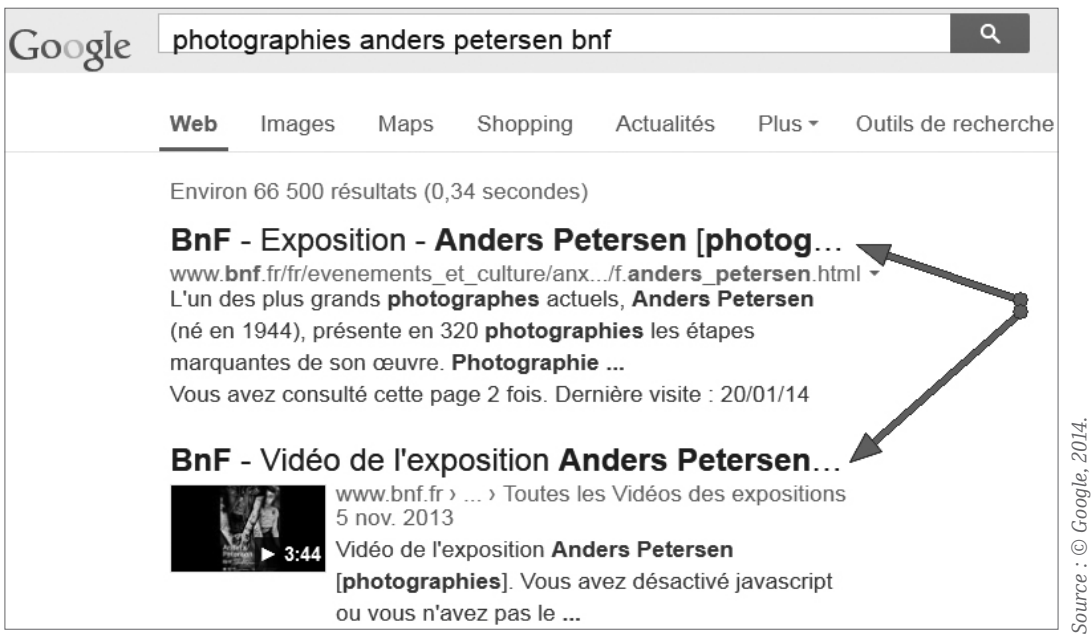

La structure est la suivante $:<$ title $>$ [Nom du site $]-[$ Rubrique $]-[$ Contenu h1] $</$ title $>$.

\section{TEXTE VISIBLE}

Il s'agit du texte affiché dans le navigateur car détecté dans le code source HTML. Afin de l'optimiser, le rédacteur met en exergue les mots-clés importants (mise en gras, titre éditorial hl, texte des liens). Il prend en compte les différentes formes de mots (singulier/pluriel, masculin/féminin) ainsi que la proximité des mots et leur ordre. Le titre éditorial est recopié dans la balise < title $>$ et dans l'URL.

Le rédacteur reste sur un indice de densité d'un mot-clé (IDM) entre $2 \%$ et $5 \%$ pour les mots-clés importants sans accorder à ce critère une importance trop grande. 
LA BALISE MÉTA DESCRIPTION APPARAÎT

CAPTURE D'ÉCRAN 4

SOUS LA BALISE < TITLE > DE LA PAGE

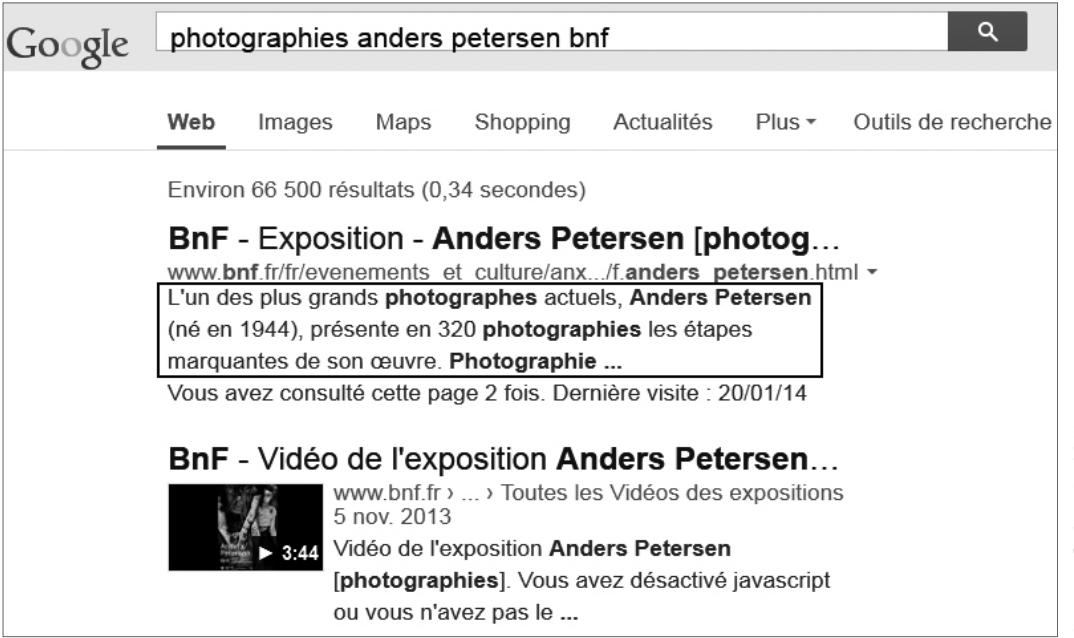

\section{L'URL DES PAGES WEB}

Il n'est pas toujours possible d'insérer des mots-clés dans l'URL selon le système d'édition utilisé dans la bibliothèque. L'idéal est que le contenu du titre éditorial $<\mathrm{hl}>$ soit recopié à la fin de l'URL et au début de la balise $<$ title $>$.

\section{LA BALISE MÉTA DESCRIPTION}

" Elle indique aux moteurs de recherche ce qu'il faut afficher pour résumer le contenu d'une page d'un site Web. Le texte entré dans cette balise sera affiché sur les snippets* de la page de recherche. ${ }^{18}$ Afin de pouvoir être affichée, cette balise est le développement du titre de la page et un résumé du contenu textuel de la page. Elle contient suffisamment de

18. Muriel Vandermeulen, « Rédiger sa métadescription, comme la petite porte d'une grande histoire ", Blog Écrire pour le web - Contenus et canaux web. [En ligne]< http://www.ecrirepourleweb.com/metadescription-petite-porte-grande-histoire >. 
caractères (150-200 caractères espaces compris). Cette taille a tendance à grandir (300 caractères).

Il est préférable d'automatiser le travail sur le contenu de ces balises. Ce travail est payant, non sur le positionnement, mais sur la façon dont les internautes perçoivent et comprennent nos pages, donc sur le taux de clics dans les Search Engine Results Page (SERP). C'est une zone " marketing qui doit donner envie aux internautes de cliquer pour venir sur le site, beaucoup plus qu'une zone de pur positionnement algorithmique de pertinence. ${ }^{19}$ Idéalement, chaque page doit proposer une balise méta description qui lui est propre.

\section{LE TEXTE ALTERNATIF DES IMAGES}

Le rédacteur devrait pouvoir entrer des mots-clés dans l'attribut alt de la balise < img/> lorsqu'il insère une image. Cette zone est prise en compte par Google, mais il est fort probable que le poids attribué dans l'algorithme de pertinence est faible.

\section{RÉDACTION DES LIENS HYPERTEXTES}

Les liens sont importants car ils permettent d'insérer des mots-clés donnant un poids plus fort à la page qui les contient (page d'origine) ainsi qu'à la part vers laquelle il dirige (page cible). Les liens hypertextes textuels sont les plus efficaces. Le rédacteur soigne particulièrement le texte du lien (le lien cliquable) en y insérant des mots-clés. On évite ainsi les liens de type Cliquez ici, Ici ou Lire la suite. 


\section{3}

\section{PANORAMA DES OUTILS DE PRODUCTION EN LIGNE}

par

Lionel Dujol

\section{UN CHOIX STRATÉGIQUE}

Le choix de l'outil de gestion et de diffusion des contenus est à penser en amont. Ce choix influera sur l'organisation interne de production et de validation des contenus ainsi que sur leurs dispositifs de diffusion et de dissémination auprès des publics visés. Il convient donc de bien qualifier ses attentes afin que cet outil soit adapté au contexte du projet.

Les solutions libres de droit présentent des avantages certains. Elles assurent une indépendance par rapport à un éditeur propriétaire. Open source, elles sont plus respectueuses des standards et plus adaptées à l'ajout de modules d'extension ou à un interfaçage avec d'autres applications. La diffusion de ce code source à une communauté d'utilisateurs est garante de la capacité à évoluer et de la pérennité de la solution. Le dynamisme de cette communauté est donc un critère de choix essentiel.

\section{LES OUTILS DE CONTENT MANAGEMENT SYSTEM}

Un système de gestion de contenus ou «CMS » est un logiciel de site Web à installer sur un serveur, disposant de fonctionnalités de publication et offrant une interface d'administration permettant de créer et d'organiser les différentes rubriques. L'un des principes clefs des CMS est d'établir une distinction entre le contenu produit par des rédacteurs et le contenant déterminé par des gabarits, des feuilles de style, une charte graphique et géré par un webmaster. Les principales solutions libres de droit sont Typo3, Drupal, Joomla ou encore Spip.

Les attentes fonctionnelles essentielles sont les suivantes : 
Les fonctionnalités liées à la gestion de contenu :

- structurer les articles à l'aide de formulaires adaptés permettant d'en guider la saisie et d'assurer ainsi leur cohérence ;

- organiser les contenus et structurer le site en arborescence, en rubriques ou en mots-clés ;

- la gestion des contributeurs et de leurs droits d'accès et d'actions : consulter, mettre à jour, valider, publier, etc. ;

- organiser un workflow et une chaîne de validation pour garder un contrôle sur les publications ;

- la programmation de la mise en ligne automatique d'un contenu à une date donnée, définir sa date limite de visibilité ;

- la gestion des versions d'un article : le brouillon, la version publiée, la version archivée...

Les fonctionnalités liées à la publication :

- la gestion des gabarits de présentation;

- la sélection de contenus selon des critères comme "les trois derniers en date », " les articles rédigés par cet auteur » et un moteur de recherche interne ;

- la syndication (RSS) et la capacité à exporter et importer des contenus à des fins de partage, en particulier sur les réseaux sociaux ;

- les statistiques du site ;

- une navigation mobile adaptée aux formats des smartphones et des tablettes ;

- des outils collaboratifs entre les contributeurs comme la messagerie électronique, un forum ou un agenda partagé. 


\section{POUR QUELS USAGES ?}

Ces outils sont particulièrement adaptés à la réalisation de portails généralistes ou de portails thématiques avec gestion d'une importante communauté de contributeurs. Citons le portail des médiathèques du SAN Ouest Provence développé avec Typo3 et interopérable avec le SIGB Koha ou encore le portail thématique de la Bibliothèque municipale de Lyon, "Point d’Actus », développé avec Spip.

\section{LES BLOGS}

Le blog est une page Web utilisée essentiellement pour la publication périodique et régulière d'actualités, de liens vers des sites Web ou de courts articles sur un sujet donné et enrichis d'éléments multimédias (image, son, vidéo). À la manière d'un journal de bord, ces billets sont publiés en ordre chronologique inversé. Un blog offre la possibilité aux lecteurs de réagir aux informations publiées, ce qui lui assure une grande réactivité.

\section{PRINCIPALES FONCTIONNALITÉS}

Un blog est un outil de CMS aux fonctionnalités de gestion et de publication des contenus moins complètes que les solutions vues précédemment. Les principales fonctionnalités focalisent sur la structuration et l'organisation des articles, et la gestion d'une petite équipe de contributeurs. Mais au fil des évolutions, le fossé entre CMS et blog se comble. Certaines solutions intègrent de plus en plus de fonctions permettant une gestion fine des droits d'accès ainsi que la personnalisation et l'organisation des contenus par page et rubrique.

\section{LES SOLUTIONS DE CRÉATION DE BLOGS}

Il est possible d'utiliser une solution en ligne ou une solution logicielle. La solution en ligne est la plus simple et la plus rapide à mettre en place. Idéale si vous ne voulez pas vous embarrasser de considérations techniques. Retenons Wordpress, Blogger ou Typepad. La solution logicielle est 
à installer sur un serveur et offre des fonctionnalités proches des CMS à l'image de Wordpress ${ }^{1}$ et DotClear.

\section{POUR QUELS USAGES ?}

En l'absence de portail institutionnel, le blog est un outil idéal pour disséminer les informations et l'actualité de la bibliothèque auprès des usagers $^{2}$. Il peut être aussi complémentaire du site de la bibliothèque en l'enrichissant de fonctions interactives tout en proposant une information alternative voire décalée, à l'image du blog de la médiathèque de Guebwiller en Alsace : « Les babillages de Frida et Paco »3.

Les blogs thématiques permettent de positionner la bibliothèque auprès des communautés d'intérêts qui vont bien au-delà des usagers de la bibliothèque. C'est ainsi que le blog $\mathrm{Bmol}^{4}$ des bibliothèques de Grenoble est perçu d'abord comme un blog thématique musical avant d'apparaître comme un blog de bibliothèque. Cela peut être aussi un blog de veille documentaire sur un sujet donné. Les blogs d'animation permettent de rendre compte d'un événement ou d'une animation régulière de la bibliothèque : catalogue en ligne d'une exposition, productions d'un concours ou coups de cœur d'un club de lecture.

\section{OUTILS DE CURATION}

Les outils de curation de contenus Web offrent une nouvelle façon d'organiser et de suivre les contenus les plus intéressants sur Internet. Ces réseaux éditoriaux participatifs s'appuient sur des utilisateurs " experts » d'un sujet, qui filtrent les informations les plus intéressantes avant de les diffuser à leurs communautés de lecteurs et d'interagir avec elles. Présentons ici deux services de curation en ligne régulièrement utilisés en bibliothèque.

1. < http://fr.wordpress.com >. Wordpress est suivi par une importante communauté active, qui fournit une impressionnante quantité d'extensions à télécharger.

2. Voir la page consacrée aux blogs de bibliothèque sur Bibliopedia < http://www.bibliopedia.fr/ index.php/Biblioblogs >.

3. < http://mediathequeguebwiller.blog.fr/ >

4. < http://bmol.bm-grenoble.fr/ >. 


\section{PEARLTREES}

Ce service Web permet d'organiser ses pages Web favorites sous forme de cartes thématiques et de les partager au sein d'une communauté d'intérêts. Chaque page Web correspond à une «perle » qui est rattachée à un « arbre à perles », l'équivalent d'un dossier.

\section{Fonctionnalités}

- éditer, retirer, ajouter des perles aux pearltrees ;

- chercher des membres et sujets via un moteur de recherche communautaire ;

- possibilité de se connecter à d'autres cartes et de les inclure au sein de ses propres arbres ;

- partager des perles et arbres à perles via Twitter, Facebook, blogs, ou par email ;

- créer un édito pour chaque arbre à perles ;

- travailler en équipe avec le suivi de l'historique des modifications apportées et restauration d'éventuelles perles/arbres effacés.

\section{Pour quels usages ?}

Proposer un dossier Web autour de sujets thématiques pour porter une expertise bibliothèque et faire équipe avec des amateurs qui partagent les mêmes centres d'intérêt ${ }^{5}$.

\section{SCOOP.IT}

Scoop.it permet à un utilisateur d'agréger les meilleurs pages du Web relatives à un sujet et de les compiler sous un format " journal ». C'est un outil à la croisée du blog, du site de partage de signets et du réseau social.

5. Voir la page des pearltrees de bibliothèques sur Bibliopedia $:<$ http://www.bibliopedia.fr/index. php/R\%C3\%A9seaux_sociaux\#Peartrees >. 


\section{Fonctionnalités}

- suggérer des contenus en fonction d'un centre d'intérêt choisi ;

- annoter les contenus partagés ;

- ajouter des pages Web au gré de sa navigation ;

- les lecteurs peuvent s'abonner à une page Scoop.it à la manière de Twitter ou via un flux RSS et partager le journal sur les réseaux sociaux ;

- les lecteurs peuvent suggérer des nouveaux contenus au créateur du journal, qui choisit ou non de les intégrer.

\section{Pour quels usages?}

Scoop.it permet de proposer une veille thématique éditorialisée et partageable ${ }^{6}$.

\section{RÉSEAU SOCIAL}

À côté des grands réseaux sociaux tels que Facebook ou Twitter, il est possible de développer sa propre plate-forme sociale avec un outil comme Elgg. Il s'agit d'un logiciel libre permettant de mettre en place un réseau social en ligne. Il fournit notamment des outils de blog, de micro-blogging, de partage de fichiers, de mise en réseau des profils d'utilisateurs, de gestion de groupes d'utilisateurs, d'agrégation de données et de nombreuses autres fonctionnalités.

\section{POUR QUELS USAGES ?}

Carnets $^{2}$ université Paris Descartes ${ }^{7}$ est une plate-forme collaborative d'expression, de réflexion et d'échanges destinée aux membres de la communauté universitaire de Paris Descartes et développée avec Elgg. Enseignants, chercheurs, étudiants, anciens élèves, l'ensemble des acteurs

\footnotetext{
6. Voir la page des Scoop.it de bibliothèques sur Bibliopedia : < http://www.bibliopedia.fr/index. php/R\%C3\%A9seaux_sociaux\#Scoop.it >.

7. < http://carnets.parisdescartes.fr/ >.
} 
de l'université peut prendre la parole sur ce réseau. Les contenus sont accessibles à tous et relayés vers d'autres réseaux tels que Facebook et Twitter. Publier des articles, partager des informations, commenter ou recommander des publications, les acteurs du réseau peuvent s'exprimer tout en valorisant leurs projets universitaires et professionnels.

Ces outils de publication sont en constante et rapide évolution. En outre, les modèles économiques ne sont pas encore stabilisés. Il n'est pas impossible que l'un d'eux cesse d'être distribué ou développé. Comme nous l'avons souligné, le choix d'un logiciel libre de droit accompagné par une communauté active d'utilisateurs est une garantie indispensable pour la pérennité de cet outil et de l'offre de contenu. Ce choix se construit donc dès la conception du projet. 


\section{MÉMENTO}

par Christelle Di Pietro

Ce mémento a pour objet de synthétiser les principales étapes de la production de contenus telles qu'elles ont été énoncées par les auteurs de cette Boîte à outils.

\section{QUELLES ÉTAPES POUR METTRE EN CEUVRE SA PRODUCTION DE CONTENUS ?}

\section{ÉLABORER UN PROJET DE SERVICE DOCUMENTAIRE ET UNE LIGNE ÉDITORIALE}

La production de contenus en ligne ne peut être conçue sans le développement d'un projet documentaire global en phase avec le projet de service de la bibliothèque.

Cela passe idéalement par la définition préalable des objectifs documentaires de la bibliothèque, donc par l'élaboration d'une charte ou d'une politique documentaire qui fixe les grands objectifs des collections de la bibliothèque.

Le projet documentaire doit fixer des objectifs qui répondent à la fois à la demande des publics et au projet politique de la collectivité, qui doit être décliné dans le projet d'établissement. Un tel projet arrive donc au bout d'une longue validation institutionnelle qui fixe de façon précise les missions de la bibliothèque dans son contexte.

Le choix de production peut être envisagé dans un cadre institutionnel, c'est-à-dire sur le site Web de la bibliothèque, ou bien selon des identités propres et techniquement autonomes (blogs ou sites externes) qui offrent plus de souplesse et moins de formalisme dans les contenus.

Le projet doit s'appuyer sur une ligne éditoriale, véritable positionnement stratégique et philosophique, qui détermine le positionnement du contenu, ses objectifs de public, les sujets abordés, le ton et le style, la périodicité, les mentions de responsabilité. C'est un document à usage externe qui est connu de tous les rédacteurs. La ligne éditoriale est pérenne, elle fixe 
un projet à moyen ou long terme de production de contenus et fédère un lectorat en accord avec ses objectifs. Sauf modifications importantes des choix institutionnels, elle ne varie pas ou peu au fil des ans.

Cette ligne est généralement déclinée dans une charte éditoriale qui définit les règles de rédaction et de validation des contenus.

\section{DÉTERMINER DES OBJECTIFS DE PRODUCTIONS ET DE PUBLICS}

Les produits eux-mêmes peuvent faire l'objet d'une concertation en équipe où chacun peut exposer ses préférences et ses options, ainsi que son expérience en la matière. Le choix des types de produits est intimement lié aux objectifs généraux du projet : valorisation des collections, réponses aux demandes documentaires, fondation d'une communauté d'usagers, information sur le fonctionnement (blog de chantier par exemple), etc. On peut aussi fixer les règles de production qui vont être reprises dans la charte éditoriale.

Les objectifs de publics doivent être fixés en fonction de ceux qui sont bien sûr définis par les missions des bibliothèques, et éventuellement recentrés sur des publics spécifiques. Souvent les services en ligne visent les publics absents, ceux qui ne fréquentent pas régulièrement la bibliothèque mais pourraient en avoir un usage. C'est le cas des publics dits " empêchés »- personnes privées de la totalité des services offerts du fait d'un handicap ou d'une impossibilité de se déplacer (encore que la définition ${ }^{1}$ de ces publics puisse être assez large en concernant ceux dont les horaires de loisir ne correspondent pas avec ceux proposés par la bibliothèque), étudiants et chercheurs qui doivent s'alimenter d'une grande quantité d'information sans avoir toujours le temps de se déplacer ou de procéder à des synthèses, ou bien encore le public qui veut confronter sa connaissance de l'actualité à des approfondissements circonstanciés.

Enfin, il est important de planifier la production tant en ce qui concerne la périodicité et la fréquence de publication que la programmation mensuelle voire annuelle. En ce qui concerne les réseaux sociaux, les créneaux horaires de publication sont importants : on constate davantage de trafic les soirs et les week-ends. Pour les dossiers en ligne, la durée de

1. Voir le glossaire Coopération des CRFCB : < http://blogs.univ-poitiers.fr/glossaire-mco >. 
publication sera une donnée importante à déterminer dès la publication : à partir d'un certain volume, la production en ligne constitue une collection, elle doit donc faire l'objet d'une politique documentaire comme l'est la collection matérielle de la bibliothèque, en particulier en matière de dé-publication/désherbage.

\section{DÉFINIR UNE CHARTE ÉDITORIALE ET UN WORKFLOW}

La ligne éditoriale permet de rédiger une charte rédactionnelle, véritable manuel de rédaction, qui garantit la cohérence des contenus. Elle comprend l'ensemble des règles et procédures en matière d'édition, de rédaction, d'illustration, de typographie.

Elle fixe aussi les processus de validation de publication des contenus, le workflow : qui sont les rédacteurs ? Que rédigent-ils ? À quel rythme ? Qui valide et publie leurs contenus ? Ces questions sont particulièrement aiguës dans le cadre d'une publication en flux, comme cela est le cas sur les réseaux sociaux. L'instantanéité doit aussi être anticipée, et les éventuels problèmes doivent être gérés de façon objective et professionnelle. La charte rédactionnelle définit également les règles en matière d'identité numérique du bibliothécaire : publie-t-il en son nom propre ou bien sous une identité professionnelle ? La bibliothèque est-elle identifiée en tant que telle ou disparaît-elle derrière l'identité du service ? La construction d'une identité numérique propre s'avère souvent indispensable pour la bibliothèque soucieuse de fédérer une communauté spécifique.

\section{CHOISIR DES OUTILS}

Le choix de l'outil arrive en fin d'élaboration du projet, il ne doit pas être choisi avant que le cadre de production ait été fixé. Il doit correspondre aux besoins et ne pas imposer un mode de fonctionnement par défaut. Le Web 2.0 a vu l'émergence d'outils ergonomiques et faciles d'utilisation, souvent gratuits, qui peuvent facilement être pris en main par des rédacteurs non familiers de techniques informatiques. Le choix peut porter sur des outils libres qui peuvent être adaptés aux besoins sous réserve de disposer d'un développeur en interne ou bien de pouvoir consacrer un budget à un prestataire externe. Les portails intégrés doivent absolument être 
évités : souvent propriétaires, ils n'offrent que très peu de souplesse et d'utilisation et imposent des formats de publication qui ne correspondent pas aux besoins des bibliothèques qui souhaitent travailler de façon partagée et collaborative tout en sécurisant les accès.

\section{ÉTABLIR UN PLAN DE FORMATION}

La production de contenu repose essentiellement sur la compétence des rédacteurs-trices qui produisent, il est donc primordial de recenser les compétences nécessaires et d'établir un plan de formation adéquat.

Deux axes sont à privilégier : la rédaction sur le Web et l'utilisation des outils. Rédiger en ligne, quelquefois sous son nom, c'est s'exposer au jugement de son public, voire de ses collègues. Il est donc important de mâ̂triser des techniques d'écriture qui permettent de cadrer la production et la systématiser. Outre la forme rédactionnelle, la production en ligne repose aussi sur une parfaite maîtrise des sources d'information. Il est essentiel de disposer d'un bagage professionnel permettant de chercher l'information pertinente, la filtrer, la synthétiser, voire la vulgariser. Cela permet d'asseoir le service sur des connaissances solides qui ne sont pas susceptibles d'être remises en cause. Établir des partenariats avec des experts thématiques peut apporter une caution scientifique solide à la production en ligne.

Même si les outils récents de production en ligne sont intuitifs, être formé permet d'acquérir des procédures de travail communes à l'ensemble de l'équipe : utiliser ou non la fonction de révision, élaborer les autorisations de publication, modification et suppression, fixer un circuit de validation. La formation à l'outil permet d'aborder par un biais technique ces questions de façon plus simple que par l'entremise d'une formalisation des procédures indépendantes.

\section{ÉVALUER POUR ÉVOLUER}

L'évaluation de la production de contenus doit idéalement être conduite de façon annuelle. Elle porte sur des éléments quantitatifs : nombre de visites et évolution, partages (sur les réseaux sociaux), commentaires, inscriptions le cas échéant ; et qualitatifs : satisfaction (mesurable par un 
questionnaire), palmarès des sujets, provenance géographique des lecteurs, analyse des mots-clés utilisés, étude ergonomique du site Web.

Cette évaluation doit être conduite dans l'optique d'une corrélation entre les objectifs poursuivis au départ et ceux réellement atteints. Le projet documentaire est-il en phase avec la politique publique mise en œuvre ? Remplit-il les objectifs définis initialement dans le cadre du service documentaire ? Il ne faut pas perdre de vue que l'évaluation se fait sur la durée, notamment pour les services proposés sur les réseaux sociaux : le temps d'appropriation par les usagers et la fidélisation d'une communauté peut prendre un temps assez long : la bibliothèque n'est qu'un pourvoyeur d'offre parmi beaucoup d'autres.

Cette évaluation doit se traduire concrètement par une évolution des services et produits proposés. La médiation d'Internet se modifie au fur et à mesure que l'aptitude des publics à satisfaire ses besoins gagne en autonomie. Tel service constitué sur la base d'un manque de compétence de son public à s'approprier un champ documentaire peut voir son intérêt décliner à cause de l'aptitude croissante dudit public à s'affranchir d'un médiateur. On peut donc affirmer que l'avenir des services documentaires en ligne tend probablement à plus de complexité et de raffinement de l'offre, en proposant des produits toujours plus élaborés à partir des compétences propres de l'établissement documentaire.

\section{SCHÉMA LES ÉTAPES DE LA PRODUCTION EN LIGNE}

Ce schéma liste les différentes étapes de la production sans forcément d'ordre chronologique entre certaines étapes. Par exemple, la phase de gestion est une étape itérative du processus, susceptible d'être conduite en parallèle de la production. 


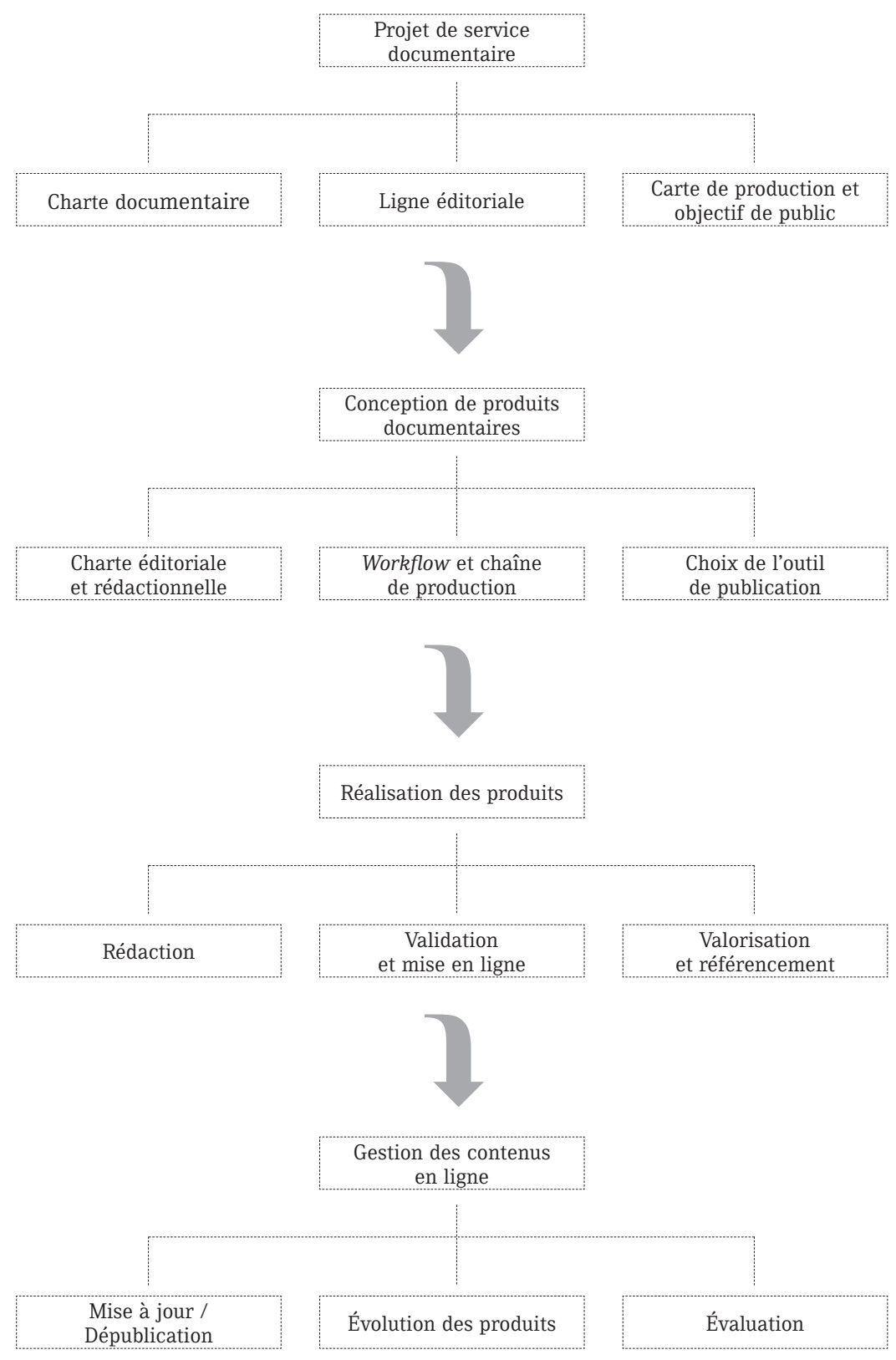




\section{SIGLES ET ACRONYMES}

ADAGP

Société des auteurs dans les arts

graphiques et plastiques

ADBU

Association des directeurs de bibliothèques universitaires

ADDNB

Association pour le développement des documents numériques en bibliothèque

BMVR

Bibliothèque municipale à vocation

régionale

CFC

Centre français d'exploitation du droit de copie

\section{CEVU}

Conseil des études et de la vie universitaire

\section{CGU}

Conditions générales d'utilisation

CLL

Centre du livre et de la lecture

CM

Community manager

CRFCB

Centre régional de formation aux carrières des bibliothèques

\section{DBIST}

Direction des bibliothèques et de l'information scientifique et technique

DRAC

Direction régionale des affaires culturelles

DRM

Digital Rights Management (gestion des droits numériques)

DSI

Diffusion sélective d'information

DTD

Document Type Definition (définition de type de document)

\section{EAD}

Encoded Archival Description (description archivistique encodée)

\section{ECTS}

European Credit Transfer System, système européen de transfert et cumul des crédits

FB

Facebook

\section{FRBR}

Functional Requirements for Bibliographic Records, spécifications fonctionnelles des notices bibliographiques. 
GED

Gestion électronique des documents

IDM

Indice de densité d’un mot-clé

IEP

Institut d'études politiques

INTD

Institut national des techniques de la documentation

\section{OAI-PMH}

Open Archives Initiative - Protocol for Metadata Harvesting (protocole pour la collecte de métadonnées de l'initiative pour les archives ouvertes)

\section{OCR}

Optical Character Recognition (reconnaissance optique de caractères)

\section{PAPE}

Plan d'action pour le patrimoine écrit

PPP

Partenariat public privé

\section{Rameau}

Répertoire d'autorité-matière encyclopédique et alphabétique unifié

\section{RSN}

Réseau social numérique

\section{SACEM}

Société des auteurs, compositeurs et éditeurs de musique
SAIF

Société des auteurs des arts visuels et de l'image fixe

\section{SERP}

Search Engine Results Page (page de résultats de recherche)

\section{SHS}

Sciences humaines et sociales

SLL

Service du livre et de la lecture

\section{SOFIA}

Société française des intérêts des auteurs de l'écrit

\section{SOMREV}

Signalement de sommaires de revues

\section{SPIP}

Système de publication pour un internet partagé

URI

Uniform Resource identifier

URL

Uniform Resource Locator

XML

Extensible Markup Language, langage de balisage extensible 


\section{GLOSSAIRE}

Affordance

Capacité d'un objet à suggérer

sa propre utilisation.

ARK

\section{(Archival Resource Key)}

Système d'identifiants qui permet d'identifier des objets de manière pérenne. Il peut s'agir d'objets de tous types, physiques (table, livre), numériques (livre numérisé...). L'ARK a vocation à se substituer aux URL et URI qui ne sont pas immuables. Pour en savoir plus : < http:// www.bnf.fr/fr/professionnels/issn_isbn_ autres_numeros/a.ark.html >.

\section{Bac à sable}

Plate-forme modélisée de tests techniques et rédactionnels préalable à la réalisation finale.

\section{Bibliobox}

Dispositif nomade issu de la Piratebox qui permet d'accéder à des ressources numériques sans connexion Internet pour échanger librement des contenus dans le respect de l'anonymat de l'utilisateur. En bibliothèque, elle permet de mettre à disposition du public des œuvres du domaine public ou des contenus créés par les bibliothécaires.

\section{Catalogue sémantique}

Catalogue consolidé à partir de données externes, qui utilise les standards du Web sémantique pour procéder à l'enrichissement des collections cataloguées avec des ressources documentaires disponibles sur le Web, notamment celles relatives aux auteurs, ou dans des réservoirs de données, comme Data BnF.

\section{Chapô}

Texte court placé au début d'un article dont il constitue l'accroche. Il peut être introductif, incitatif ou rappeler un contexte.

\section{Content Management System (CMS)}

Système de gestion de contenu, logiciel qui permet de gérer et mettre à jour les pages d'un site Web.

\section{Community manager}

(gestionnaire de communauté)

Animateur de communautés en ligne.

\section{Curation de contenus}

Sélection, édition et partage de contenus en ligne sur une thématique.

\section{Délit de presse}

Crimes et délits commis par voie de presse ou tout autre moyen de publication. Par exemple : la provocation aux crimes et délits, la contestation de crimes contre l'humanité, la diffamation, l'injure, etc.

\section{Document primaire,} secondaire, tertiaire

Le document primaire est l'œuvre originale telle que conçue par son auteur, le document secondaire est un signalement ou une description de la première (une notice ou une critique par exemple). Le document tertiaire est un produit documentaire qui condense plusieurs documents sous forme d'une synthèse.

\section{Epub (Electronic Publication,} publication électronique)

Format ouvert standardisé pour les livres numériques. Epub3 est la dernière version qui permet d'ajouter des contenus enrichis (multimédia). 
Fedora (Flexible Extensible Digital Object Repository Architecture)

Outil de dépôt, de gestion et de mise en valeur d'objets numériques, développé en Open source par des chercheurs de l'Université Cornell.

\section{Front Office (logiciel)}

Partie du logiciel qui est accessible directement par l'usager final.

\section{Geemik}

Mot-valise inventé par les animatrices de communautés de l'École supérieure de commerce de Lille (Skema). Il est composé de " geek » signifiant férue de nouvelles technologies, et " gimmick » un terme de jazz désignant les cinq premières notes qui donnent le ton, pour les cinq animatrices.

\section{Influenceur, ou e-influenceur}

De l'anglais influencer, désigne originellement une personne au sein d'une entreprise en charge de construire ou défendre la réputation d'une marque sur le Web, en particulier sur les réseaux sociaux. Par extension, ce sont les prescripteurs et leaders d'opinion en ligne, à titre professionnel ou non, susceptibles d'influencer le comportement des internautes.

\section{Longue traîne}

Concept développé en 2004 par Chris Anderson pour décrire un marché, ou des usages, qui s'étendent sur plusieurs produits en petites quantités, la somme totale représentant une part importante de la totalité du segment. Appliqué à l'indexation Web, c'est l'ensemble des mots-clés qui génèrent chacun peu de trafic mais dont le cumul représente plus que la somme des mots-clés les plus représentés.

\section{Marronnier}

Dans le jargon journalistique, sujet qui revient de façon cyclique dans l'actualité, comme la rentrée des classes ou les bouchons des routes des vacances.

\section{METS (Metadata Encoding and Transmission Standard)}

Format maintenu par la Bibliothèque du Congrès qui sert à exprimer diverses métadonnées décrivant un document numérique, dans le but de faciliter son échange, sa gestion et sa préservation, voir le portail de la $\mathrm{BnF}:<$ http://www.bnf.fr/ fr/professionnels/formats_catalogage/a.f_ mets.html >.

\section{Microblog}

Blog aux billets très courts (ex : Tumblr).

\section{MODS (Metadata Object Description Schema)} Schéma XML de description bibliographique. Il a été initialement développé par la Bibliothèque du Congrès pour la conversion sans perte de notices bibliographiques en MARC. Pour en savoir plus : < http://www.loc.gov/standards/mods/v3/ mods-3-5.xsd $>$.

\section{Moodle}

Plate-forme d'apprentissage en ligne sous licence libre.

\section{Open source (code source ouvert)}

Désigne les logiciels dont la licence est en libre redistribution et dont le code de programmation est ouvert et modifiable.

\section{Produit de synthèse}

Voir document tertiaire.

\section{QR code (Quick Response)}

Code-barres en deux dimensions constitué de pixels noirs disposés dans un carré 
à fond blanc. Il permet de stocker des informations numériques (textes ou adresses de site Web), et peut être lu par un smartphone ou une tablette.

\section{Resource Description}

\section{Framework (RDF)}

Format de description des données, par exemple celles qui existent sur le « Web de données » (les données exposées sur le Web).

\section{Revue de presse}

Synthèse des titres d'articles de différents médias sur un même sujet.

\section{Snippet}

Portion de code de programmation d'un logiciel réutilisable.

\section{SPARQL (SPARQL Protocole} And RDF Query Language)

Langage de requêtes similaire à celui employé pour faire des requêtes sur des bases de données (Structured Query Language, SQL). Il permet les interrogations de ressources au format RDF.

\section{Stopping power (" pouvoir d'arrêt ")} Désigne originellement la capacité d'une arme (à feu) à stopper un adversaire dès le premier impact. Par analogie, désigne la capacité d'un message, publicitaire en particulier, à accrocher l'attention de son destinataire.

\section{Uniform Resource Identifier (URI)} et Uniform Resource Locator (URL) Chaînes de caractère précisant la localisation d'une ressource sur le Web.
Web Ontology Language (OWL)

Langage de représentation des connaissances construit sur le modèle de données de RDF. Il permet de définir des ontologies Web structurées.

\section{Webmagazine}

Ou webzine ou e-magazine, magazine publié en ligne sans équivalent imprimé. Il est alimenté par plusieurs contributeurs, professionnels ou non, et dispose d'une ligne éditoriale.

\section{Workflow (flux de travail)}

Processus au cours duquel des tâches, des documents et des informations sont traités successivement par un groupe de personnes, selon des règles prédéfinies, en vue de réaliser un produit ou de fournir un service. Ici, il s'agit du processus de publication et de validation rédactionnelle en ligne.

$5 \mathrm{~W}+\mathrm{H}$

Acronyme anglo-saxon pour: Who, What, Where, When, Why + How, traduction du français QQOQCCP, soit: Qui, Quoi, Où, Quand, Comment, Combien, Pourquoi. Permet de systématiser le questionnement préalable à toute démarche d'analyse afin de collecter les données nécessaires à un état des lieux ou à la restitution d'un problème. 


\section{BIBLIOGRAPHIE ${ }^{1}$}

Agnès Yves, Manuel de journa-

lisme : écrire pour le journal, $2^{\mathrm{e}}$ éd., Paris, Éditions La Découverte, 2008 (coll. Grands Repères, Guides).

Andrieu Olivier, Réussir son référencement Web, Paris, Eyrolles, 2013.

Bassoni Marc, « Les pratiques documentaires des journalistes à l'heure des nouveaux médias : une rupture programmée », in Broudoux Évelyne et Chartron Ghislaine (dir.), Enjeux politiques du document numérique : actes de la troisième conférence Document numérique et société, Paris, ADBS Éditions, 2010 (coll. Sciences et techniques de l'information).

Battisti Michèle, «Et si le droit de citation était élargi aux images ? » Paralipomènes, $1^{\mathrm{er}}$ avril 2012. [En ligne] : <http://www.paralipomenes. net/archives/7726>.

Breuil Isabelle, « La formation des usagers à Paris 8 : au croisement des usages », in Liquète Vincent (dir.), Du CDI à la bibliothèque universitaire : former les usagers à l'information, Les Cahiers d'Esquisse, janvier 2010, n 1, pp. 63-69. [En

1. Ces éléments bibliographiques complètent les références citées dans les notes de bas de page. Sont ici privilégiées les références les plus récentes. ligne] : < http://www.calameo.com/ books/00017502177c37b07c0d0 >.

Calenge Bertrand, « La solubilité du bibliothécaire ", Carnet de notes, 27 août 2008. [En ligne] : < http://bccn. wordpress.com/2008/08/27/la-solubilite-du-bibliothecaire/>.

Canivet Isabelle, Hardy Jean-Marc, La stratégie de contenu en pratique : 30 outils passés au crible, Paris, Eyrolles, 2012 (coll. Design web).

Coulon Alain (dir.), L'évaluation des enseignements de méthodologie documentaire à l'université de Paris VIII, Saint-Mandé, Association internationale de recherche ethnométhodologique, 1993.

Dunyach Jean-Claude, « Comment transformer un document électronique simple (Word ou autre) en livre numérique (EPUB, MOBI...) », Blog de JeanClaude Dunyach, 20 mars 2014. [En ligne] : <http://jean-claude.dunyach. pagesperso-orange.fr/Ebooks_files/ Comment_fabriquer_un_livre_numerique.pdf>.

Galaup Xavier (dir.), Développer la médiation documentaire numérique, Villeurbanne, Presses de l'enssib, 2012 (coll. La Boîte à outils ; 25). [En ligne] : $<$ http://mediationdoc.enssib.fr >. 
Gros Nicolas, Guinard Pierre, « Numelyo, la bibliothèque numérique de Lyon », Bulletin des bibliothèques de France, 2013, n 5, pp. 12-15. [En ligne] : < http://bbf.enssib.fr/consulter/ bbf-2013-05-0012-002 >.

Haettiger Magali, « Numérisation de masse et pratiques professionnelles : l'exemple de la Bibliothèque municipale de Lyon », DocumentalisteSciences de l'information, 2010, vol. 47, $\mathrm{n}^{\circ} 2$, pp. 50-51.

Kissane Erin, Stratégie de contenu Web, Paris, Eyrolles, 2011 (coll. A Book Apart ; 3).

Maucorps Vincent, « Gestion de contenus. Le meilleur des solutions open source », Smile, 2013. [En ligne] : < http://www.smile.fr/Livres-blancs/ Gestion-de-contenu-et-ged/Les-cmsopen-source $>$.

Mercier Silvère, " Médiation numérique et culture de l'information : repositionner les bibliothécaires ", Bibliobsession, 19 décembre 2011. [En ligne] : <www.bibliobsession. net/2011/12/19/mediation-numeriqueet-culture-de-linformation-repositionner-les-bibliothecaires/>.

Nuttin Guillaume, "Bibliothécaires producteurs de contenus ", Archimag, 2012, n² 254, p. 15.

Pigeon-Bormans Anne, " Le critère de l'originalité ou les limites de la protection par le droit d'auteur »,
Avocats Publishing, juin 2013.

[En ligne] : < http://www.avocats-publishing.com/Le-critere-de-l-originalite-ou-les >.

Puybonnieux Aurélie, Se former à l'ère du numérique : nouveaux enjeux et nouveaux acteurs de la formation à l'information dans les bibliothèques universitaires. Le cas de l'Université de Paris 8, Mémoire de master : chef de projet en ingénierie documentaire, Institut national des techniques de la documentation, 2010. [En ligne] : < http:// memsic.ccsd.cnrs.fr/docs/00/57/50/61/ PDF/PUYBONNIEUX.pdf >.

Robin Christian, L'angle journalistique : techniques de créativité pour des écrits originaux, Paris, CFPJ Éditions, 2009.

Sepetjan Sophie, « Respecter le droit de la propriété littéraire et artistique », in Claerr Thierry et Westeel Isabelle (dir.), Numériser et mettre en ligne, Villeurbanne, Presses de l'enssib, 2010 (coll. La Boîte à outils ; 19).

Sivan Isabelle, « Quand une œuvre tombe-t-elle dans le domaine public ? », Sivan Avocats, 20 février 2012. [En ligne] : < http://www.sivanavocats.com/Domaine-public.html >.

Springer Michelle, Dulabahn Beth, Michel Phil, Natanson Barbara, Reser David, Woodward David, et Zinkham Helena, For the Common Good: The Library of Congress Flickr Pilot Project, New-York, Library of Congress, 2008. 


\section{LISTE DES ILLUSTRATIONS}

Encadré. Le circuit de production

Encadré. La charte éditoriale des brèves de l'enssib

Schéma. Structuration d'une brève

Encadré. L'interface Web publique de Sign@l

Capture d'écran. Les principales branches d'Arbradoc

Figure. Le processus global de requête

Capture d'écran. Catalogue en ligne de la bibliothèque de Fresnes

Exemple de l'introduction du dossier "Street art"

Exemple du dossier « Voyager avec ses enfants »

Exemple du dossier " La chanson française des années 2000 »

Exemple du dossier

«Camus l'intime»

Exemple du dossier

« Le Web en cuisine »

Exemple du dossier

" Il était une fois le Japon»

Exemple du dossier "Street art"

Exemple du dossier

« Réviser son BAC »

Exemple du dossier « Des molécules

dans nos assiettes »

Exemple du dossier « Français langue étrangère »

Encadré. Dossier documentaire : les étapes de production
Encadré. Netvibes, Pearltrees,

Scoop.it

Encadré. Le cadre du contrat

Google

Encadré. Les étapes de la chaîne opératoire pour la numérisation

Ego, autoportrait d'Eugène Trutat dans l'atelier Vidal, vers 1860

Encadré. Le mémo du publiant

Encadré. Exemples de publications sur les pages FB de l'UVSQ

Encadré. 10 idées à adapter aux bibliothèques

Encadré. Un exemple de planning conversationnel pour une exposition (Centre Pompidou)

Encadré. Les principales étapes de la fabrication d'un livre numérique

Encadré. Le Plan d'action pour le patrimoine écrit

Capture d'écran. Vérification de l'intérêt ou potentiel du mot-clé dans l'outil de planification des mots-clés Google

Capture d'écran. Vérification de la concurrence du mot-clé dans le moteur de recherche Google

Capture d'écran. Reprise de la balise $<$ title > par le moteur Google

Capture d'écran. La balise méta description apparaît sous la balise $<$ title $>$ de la page

Schéma. Les étapes de la production en ligne 


\section{INDEX DES OUTILS CITÉS}

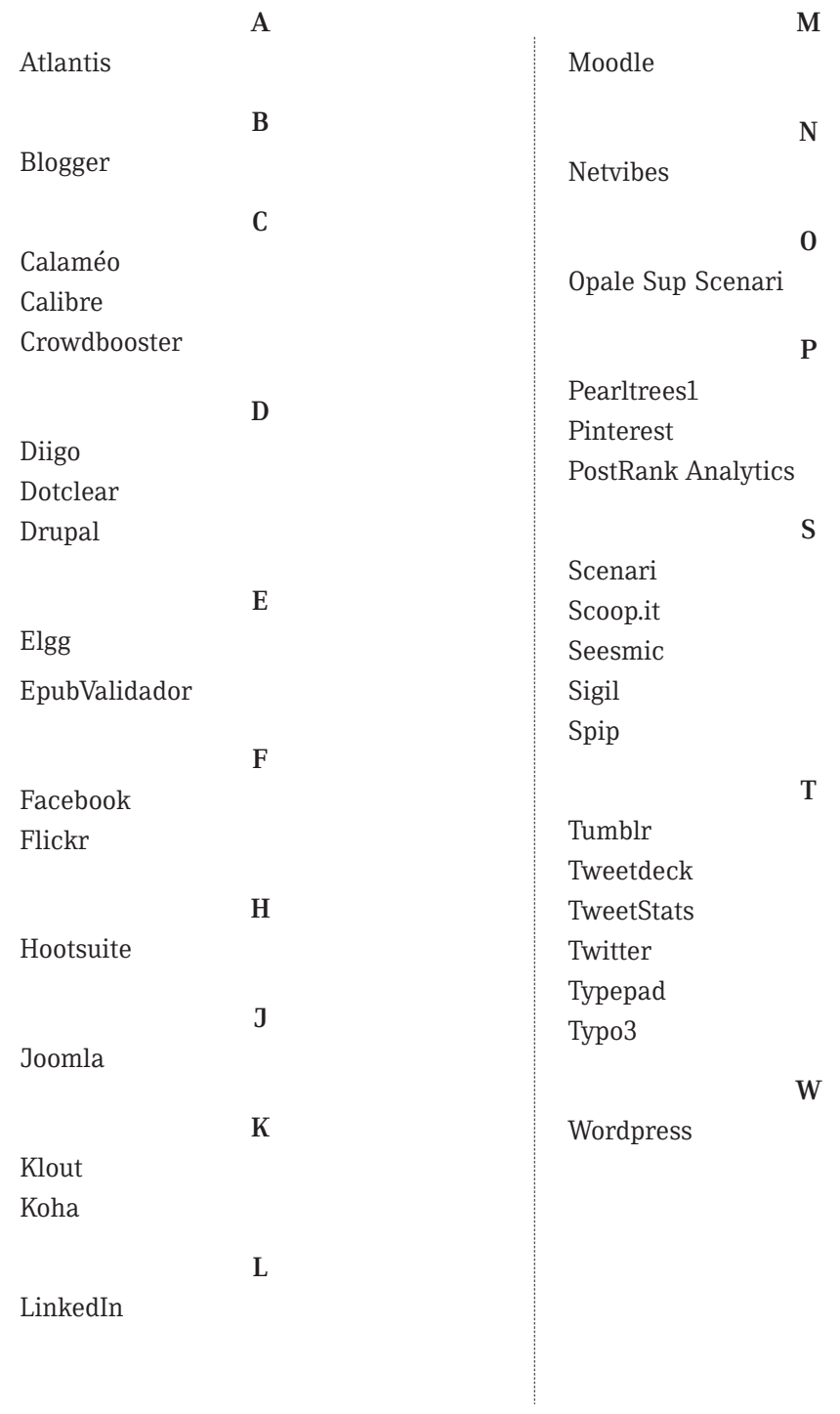




\section{LISTE DES AUTEURS}

\section{Michèle Battisti}

Rédactrice en chef de la revue Documentaliste-Sciences de l'information pour l'Association des professionnels de l'information et de la documentation (ADBS) et auteur du blog Paralipomènes < http://www.paralipomenes.net > (Paris)

\section{Pierre Bournerie}

Développeur et responsable du portail de la Bibliothèque municipale de Fresnes

\section{Isabelle Breuil}

Chargée de mission Enquête des usagers, Bibliothèque universitaire de Paris 8 Saint-Denis

\section{Jocelyne Deschaux}

Directrice du Réseau des médiathèques de l'Albigeois

\section{Christelle Di Pietro}

Conservatrice territoriale des bibliothèques, enssib - Cnfpt

\section{Lionel Dujol}

Responsable des services numériques et de la médiation numérique, Médiathèques du Pays de Romans (Romanssur-Isère)

\section{Bruno Essard-Budaïl}

Chargé de mission bibliothèques

\& patrimoine écrit, Centre du livre et de la lecture en Poitou-Charentes (Poitiers)

\section{Magali Haettiger}

Directrice du Réseau des médiathèques de Lorient

\section{Patrick Hernebring}

Responsable du Fonds musical ancien, Service du Patrimoine écrit, Bibliothèque d'étude et du patrimoine (Toulouse)

\section{Gonzague Gauthier}

Chargé de projets numériques, Centre Pompidou (Paris)

\section{Magalie Le Gall}

Responsable des services aux publics, Bibliothèque universitaire de Versailles, Direction des bibliothèques et de l'information scientifique et technique de l'université de Versailles Saint-Quentin-en-Yvelines

\section{Dominique Macé}

Responsable du secteur adultes, Médiathèque de Bagnolet

\section{Sébastien Magro}

Chargé de projet nouveaux médias, Musée du quai Branly (Paris)

\section{Nathalie Nosny}

Chargée d'études documentaires principale, responsable du service Webmagazine, Bibliothèque publique d'information (Paris)

\section{Jérôme Pouchol}

Directeur de la politique documentaire, Médiathèque intercommunale Ouest Provence (Miramas), maître de conférences associé à l'université Pierre MendèsFrance (Grenoble)

\section{Isabelle Rouquet}

Formatrice indépendante auprès des bibliothèques publiques françaises et professeure associée, responsable de la licence professionnelle " Métiers de l'édition, des bibliothèques et du commerce du livre », université Blaise Pascal, département Métiers de la culture (Clermont-Ferrand)

Carole Tilbian

Directrice de la Bibliothèque Sciences Po Lyon 
Secrétariat d'édition:

Silvia Ceccani

Mise en page:

Cédric Vigneault

Conception graphique:

atelier Perluette, 69001 Lyon.

< http://www.perluette-atelier.com >

Achevé d'imprimer en octobre 2014

imprimerie Bialec (Nancy)

dépôt légal: $2^{\mathrm{e}}$ semestre 2014 


\section{LA BOÎTE À OUTILS}

Les manuels de cette collection visent à fournir aux professionnels des ouvrages pratiques pour conduire des projets bibliothéconomiques d'actualité concernant aussi bien des bibliothèques publiques que des bibliothèques universitaires ou de recherche. Écrit à plusieurs mains, chaque volume est coordonné par un professionnel des bibliothèques.

Catherine Jackson directrice de la collection

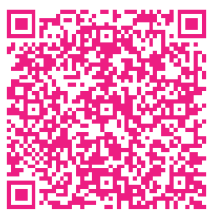

$+++++++++++++++++++++$

PRESSES DE L'enssib

École nationale supérieure des sciences de l'information et des bibliothèques

17-21 boulevard du 11 novembre 1918 69623 Villeurbanne Cedex

Tél. 0472444343

Fax 0472444344

< http://www.enssib.fr/presses >
Dans la même collection

$++++++++++++++++++++++++++++++++++++$

BA0 \#21 (2010)

Communiquer! Les bibliothécaires, les décideurs et les journalistes

sous la direction de Jean-Philippe Accart

BA0 \#22 (2011)

Mener l'enquête! Guide des études de publics en bibliothèque

sous la direction de Christophe Evans

BA0 \#23 (2011)

Créer des services innovants. Stratégies et répertoire d'actions pour les bibliothèques sous la direction de Marie-Christine Jacquinet

BA0 \#24 (2011)

Mener un projet international: bibliothèques françaises et coopération internationale sous la direction de Raphaëlle Bats

BA0 \#25 (2012)

Développer la médiation documentaire numérique sous la direction de Xavier Galaup

BA0 \#26 (2012)

Apprendre à gérer des collections patrimoniales en bibliothèque sous la direction de Dominique Coq

BA0 \#27 (2012)

Faire connaître et valoriser sa bibliothèque : communiquer avec les publics sous la direction de Jean-Marc Vidal

BA0 \#28 (2013)

Favoriser l'insertion professionnelle et l'accès

à l'emploi: les atouts des bibliothèques sous la direction de Georges Perrin

BA0 \#29 (2014)

Intégrer des ressources numériques dans les collections

sous la direction de Géraldine Barron et Pauline Le Goff-Janton

À paraître

$++++++++++++++++++++++++++++++++++++$

BA0 \#31 (2014)

Ouvrir plus, ouvrir mieux : un défi pour les bibliothèques

sous la direction de Georges Perrin 